\title{
A KINETIC STUDY OF ANTI-VEGF-A POLYCLONAL ANTIBODIES AND ANTI-VEGF-A ssDNA APTAMERS
}

\author{
A Thesis \\ presented to \\ the Faculty of California Polytechnic State University, \\ San Luis Obispo
}

In Partial Fulfillment

of the Requirements for the Degree

Master of Science in Engineering, with Specialization in Bioengineering

by

Heather Hedeen

June 2012 
(C) 2012

Heather Hedeen

ALL RIGHTS RESERVED 


\section{COMMITTEE MEMBERSHIP}

TITLE:

AUTHOR:

DATE SUBMITTED: June 2012
A Kinetic Study of anti-VEGF-A Polyclonal Antibodies and anti-VEGF-A ssDNA Aptamers

Heather Hedeen

COMMITTEE CHAIR: David Clague

COMMITTEE MEMBER: Dan Walsh

COMMITTEE MEMBER: Yarrow Nelson 
Abstract

A Kinetic Study of anti-VEGF-A Polyclonal Antibodies

and anti-VEGF-A ssDNA Aptamers

Heather Hedeen

A new detection reagent that could possibly augment or replace antibodies research and diagnosis methods are aptamers. Aptamers are ssDNA, RNA or polypeptide constructs that function like active antibodies. Antibodies and aptamers both specifically bind to selected target molecules, and as such they enable the detection or targeting of the presence or absence of a specific antigen.

In order to ensure that sSDNA aptamers perform similarly to antibodies, anti-VEGF-A polyclonal antibody and anti-VEGF-A ssDNA aptamer were evaluated against vascular endothelial growth factor A (VEGF-A) using Surface Plasmon Resonance (SPR). It was hypothesized that the anti-VEGF-A aptamer had the same, if not better, binding kinetics than the anti-VEGF-A polyclonal antibody, and as such offers an ideal replacement for use in in field, real-time testing assays.

SPR revealed that both the polyclonal antibody and SSDNA aptamer bound the target antigen, VEGF-A. Additionally, from the SPR kinetic analysis, the anti-VEGF-A aptamer had $K_{D}$ values of 20-28 nM and the anti-VEGF-A antibody had $K_{D}$ values of 16-127 UM. The binding efficacy of the aptamer was several orders of magnitude better than that of the antibody. The aptamer was also stable in solution for a longer amount of time than the antibody, which denatured in solution after two weeks.

Goals of the thesis:

- To gain an understanding of SPR instrument use

- Kinetic analysis of anti-VEGF-A polyclonal antibody

- Kinetic analysis of anti-VEGF-A ssDNA aptamer

- Ramifications of kinetic analysis 


\section{TABLE OF CONTENTS}

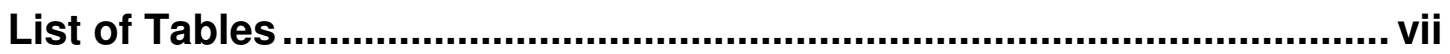

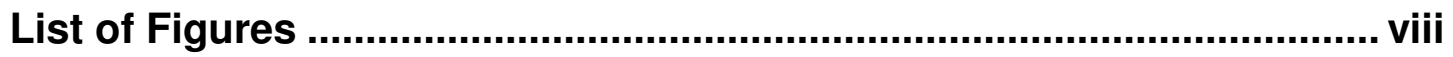

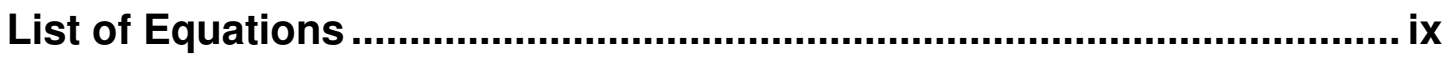

\section{Chapter}

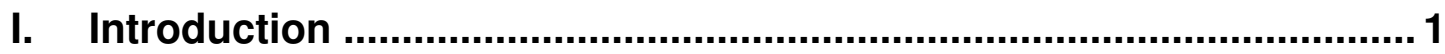

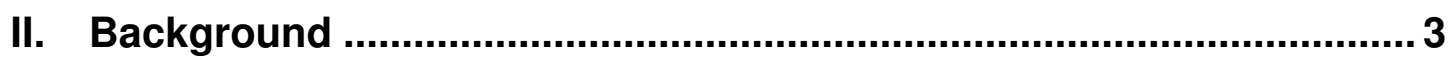

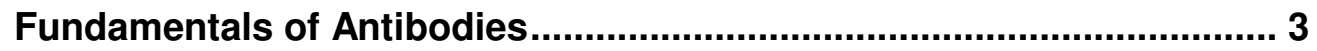

Structure and function ............................................................ 3

Monoclonal antibodies …........................................................ 4

Polyclonal antibodies ................................................................... 8

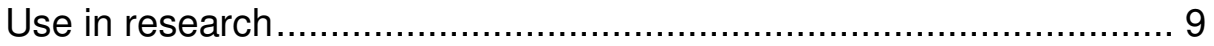

Advantages/disadvantages .......................................................

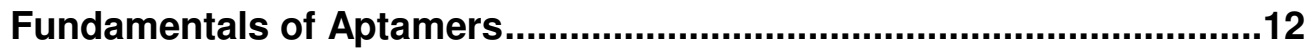

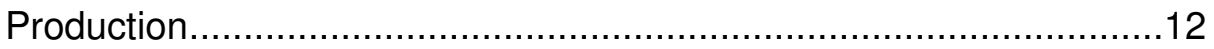

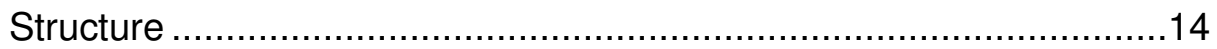

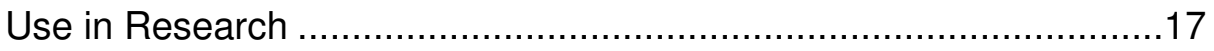

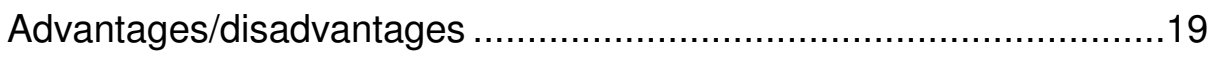

Fundamentals of Surface Plasmon Resonance (SPR) ..........................21

Surface Plasmon Resonance History and Theory .............................21

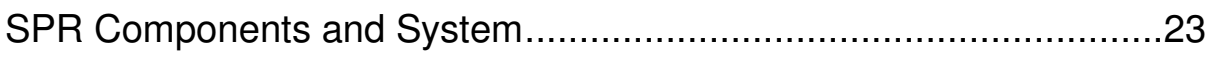

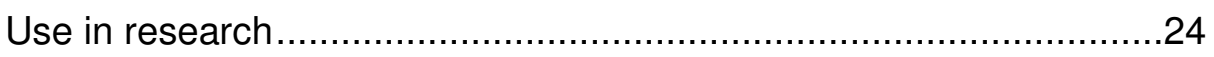

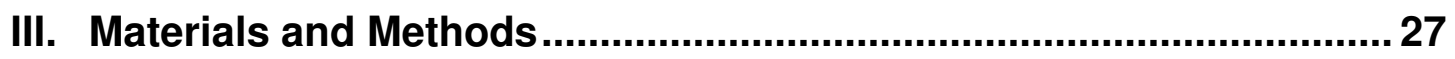

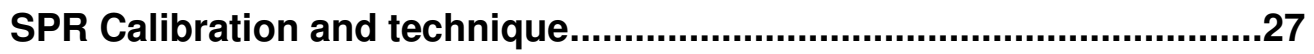

Selection of Carboxymethyl Dextran.......................................................29

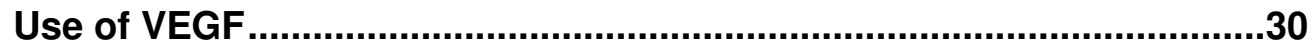

Immobilization of VEGF and antibody/aptamer interactions .................31

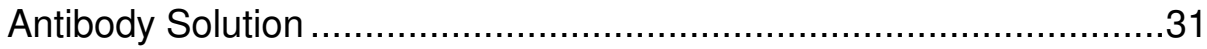

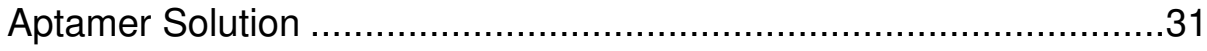

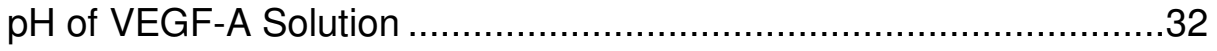


Other solutions ............................................................33

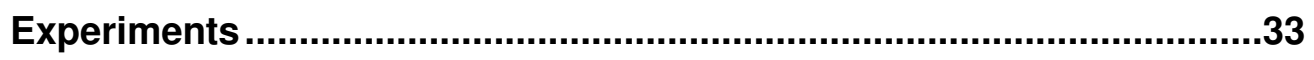

Analysis Software.........................................................................36

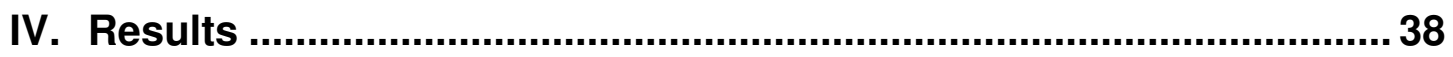

Ethanol Dilutions ....................................................................................38

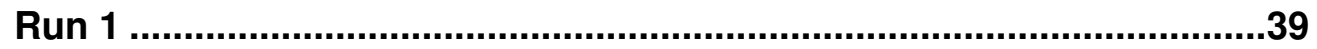

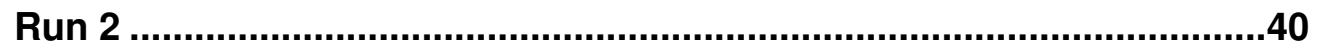

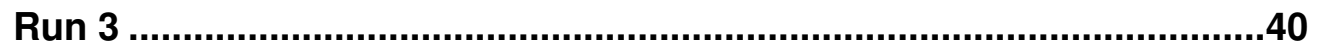

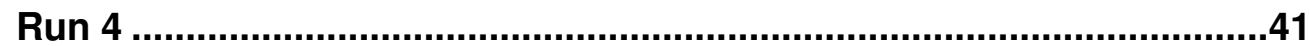

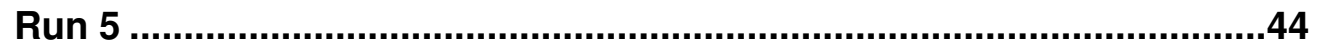

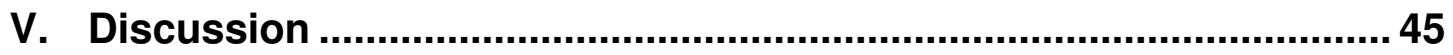

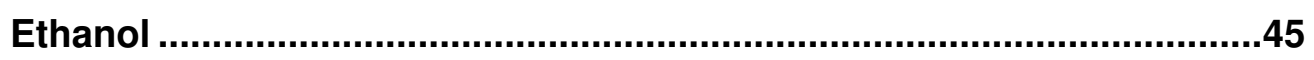

VEGF-A and anti-VEGF-A polyclonal antibodies ssDNA aptamer.........47

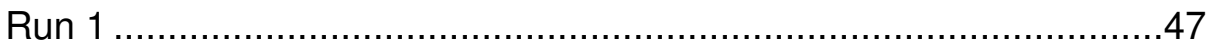

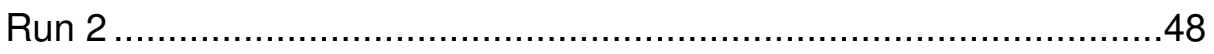

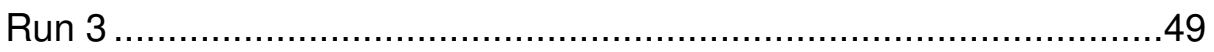

Antibody verses aptamer ......................................................... 53

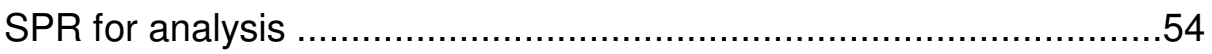

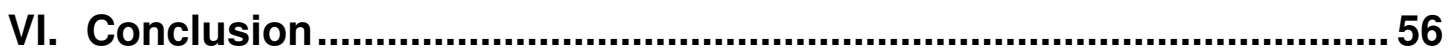

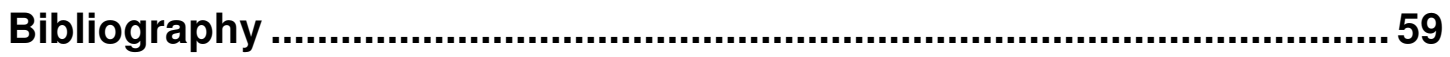

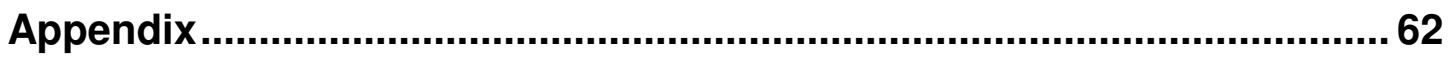

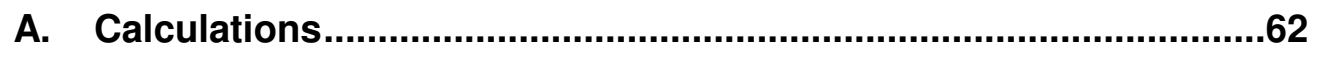

B. SPR Ethanol Raw Data...........................................................64

C. SPR Run 1 Raw Data..................................................................70

D. SPR Run 2 Raw Data.....................................................................72

E. SPR Run 3 Raw Data ..............................................................73

F. SPR Run 4 Raw Data ................................................................79

G. SPR Run 5 Raw Data..................................................................85

H. VEGF Information Sheet .................................................................92

I. Antibody information Sheet ...........................................................93

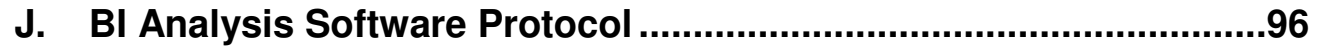




\section{LIST OF TABLES}

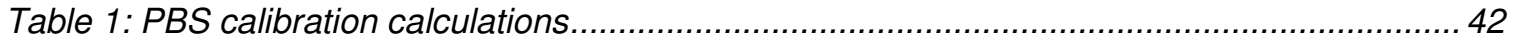

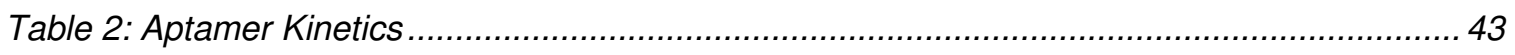

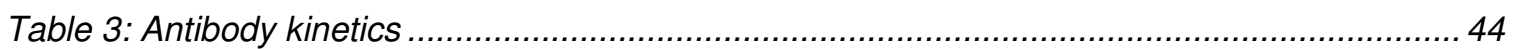




\section{LIST OF FIGURES}

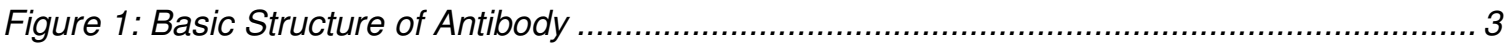

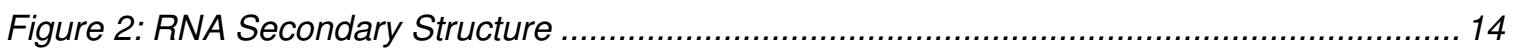

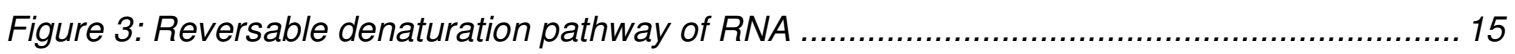

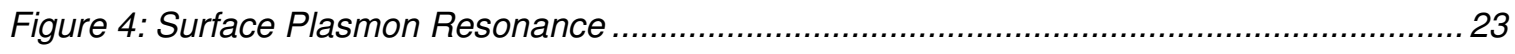

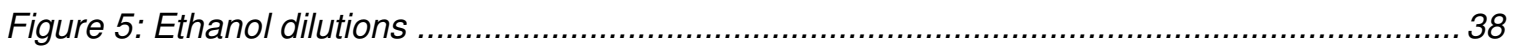

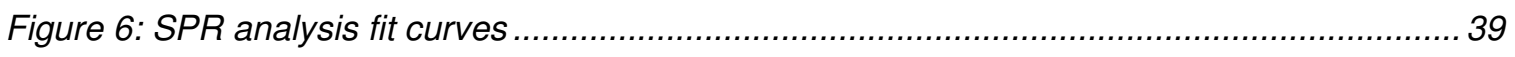

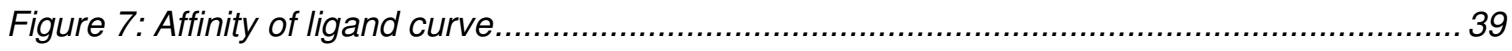

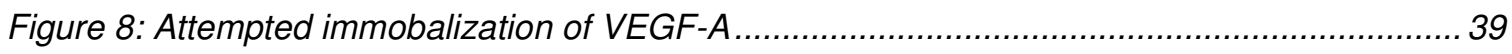

Figure 9: Absence of VEGF-A binding between antibody and aptamer ...................................... 40

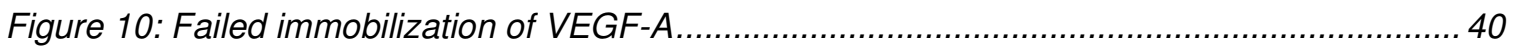

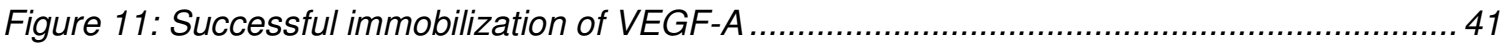

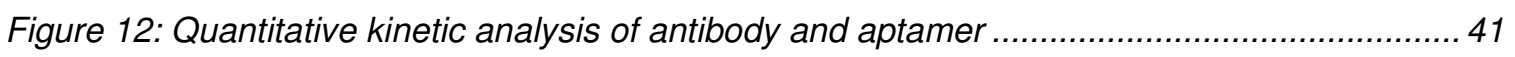

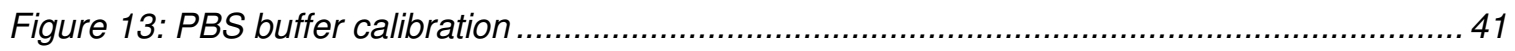

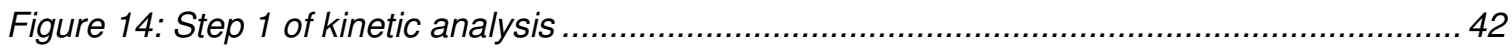

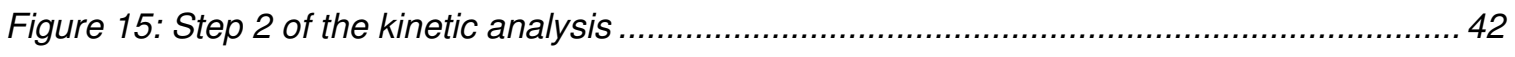

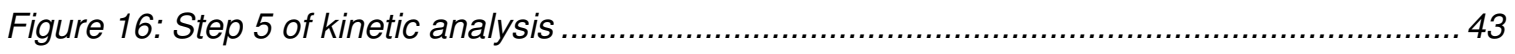

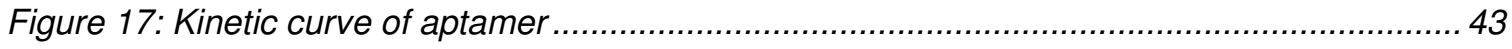

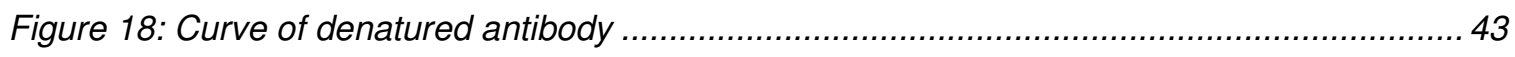

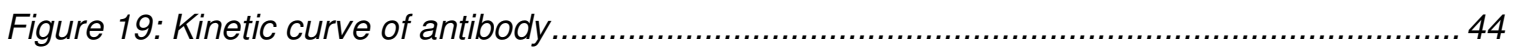

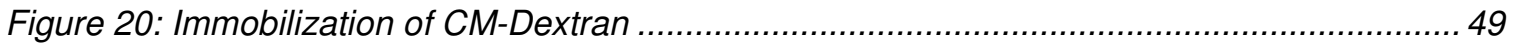

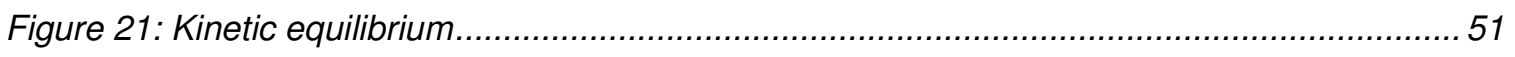

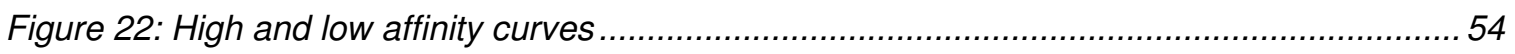




\section{LIST OF EQUATIONS}

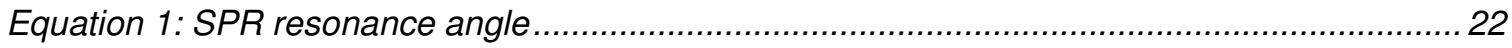

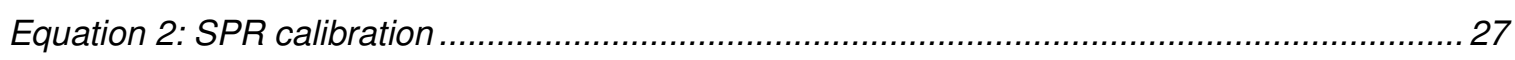




\section{INTRODUCTION}

Biotechnology is a constantly changing field of technical knowledge as new techniques and materials are researched and developed. What was once the standard in many areas of research is now giving way to these new methods and materials that are being created by researchers. Many of these new techniques allow for novel diagnostic and analysis procedures that are more cost effective and less time consuming than their predecessors. One such shift is that from antibodies to aptamers; aptamers offer a new methodology for testing and identifying selected analytes. Another advancement in detection technology in the last decade is Surface Plasmon Resonance (SPR), which requires a limited amount of antigen, even down to the order of femto-moles. However, the lowest detectable level is often dependent on the amount of background noise created and varies from experiment to experiment based on the molecules used [1].

The research presented here combines aptamers, antibodies, and SPR as an analysis technique to support the validity for shifting from antibodies to aptamers for use as detection reagents on medical and pathological diagnostic platforms. SPR is used to analyze the similarities or differences in the kinetics binding between anti-VEGF-A antibodies, anti-VEGF-A aptamers and VEGF-A. It is hypothesized that that aptamers have similar or better binding kinetics than antibodies do and as such offer ideal replacements as biotechnology seeks to produce better in field, real-time testing assays. All together the data gathered will be used to help develop a proof-of-concept for utilizing aptamers on 
diagnostic paper microfluidic testing platforms as an inexpensive, stable and reusable platform that may be performed quickly and easily.

These aptamer detection platform assays will not only affect laboratory techniques but will also allow for the production of rapid in-field testing assays for use by the FDA, first responders, the HSO, the Navy, and in developing countries. 


\section{BACKGROUND}

\section{FUNDAMENTALS OF ANTIBODIES}

\section{Structure and function}

Antibodies play a crucial role in the immune system; they are naturally produced by B-cells as part of the humoral immune response to the presence of foreign antigens within the body. The structure of antibodies has been determined through X-ray crystallography to consist of three globular regions assembled into a Y-shape. This $\mathrm{Y}$-shape is composed of two heavy and two light chains. The heavy chains are linked together by disulfide bonds into the preliminary $\mathrm{Y}$-shape and the two light chains are in turn linked to the heavy chains at the top of the $Y$ by disulfide bonds. Together the light and heavy chains make up the symmetrically identical Y-shaped antibody structure. Antibodies make up the most diverse class of proteins with all antibodies having a constant c-terminus, at the bottom of the $\mathrm{Y}-$

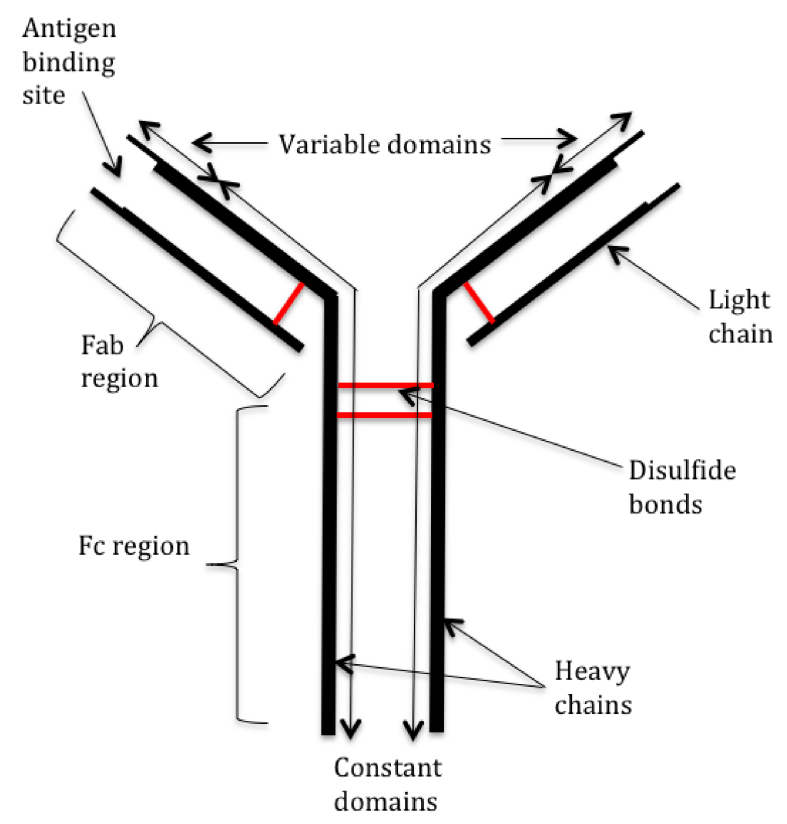

Figure 1: Basic structure of an antibody. shape, and a variable $n$ - terminus, found at the top of the $\mathrm{Y}$. The $\mathrm{n}$-terminus changes for optimal binding to antigens and is unique to each antibody produced. When antibodies are exposed to pepsin, they cleave into Fab and Fc fragments. The n-terminus is made up of the Fab fragment; it is composed of a complete 
light chain and part of the heavy chains on each side. Fab stands for fragments of antigen binding and is where the antigen interacts and binds to the antibody. The c-terminus is composed of the Fc fragment, or Fragment crystallizable fragment, made up by the bottom portion of the heavy chains. The Fc fragment does not interact with antigen, but instead interacts with cells and effector molecules. Genetic modification can be used to optimize the Fab and Fc fragments for binding and interaction between the antibody, antigen and cell receptors [2]. Antibodies that are produced for research result from two different production techniques, which produce slightly different antibody products: monoclonal and polyclonal antibodies.

\section{Monoclonal antibodies}

Niels Jerne, Georges Kohler, and Cesar Milstein developed monoclonal antibody (mAb) production and won the Nobel Prize in Physiology or Medicine in 1984 for their discovery [3]. Monoclonal antibodies are produced from cell lines created from murine derived cells that have been immunized with the desired antigen. Cells are either produced through tissue culture or direct injection of mice from which the mAb will be processed and purified. mAb production in mice is a commonly used technique that is well understood, their use and well being must monitored due to Institutional Animal Care and Use Committees. Cell culture, while becoming more common, is still not as widely understood and using in vivo techniques and is expensive and time consuming.

mAb production in mice follows five basic steps. Step 1: the mice are immunized with antigen emulsified Freud's adjuvant to encourage an immune 
response and the production of antibodies at the injection site. The mice are immunized every few weeks in order to obtain the desired antibody titer within their serum. Step 2: the mice are screened for antibody production by using blood samples to measure the level of antibodies in their serum. If the titer in a mouse is too low, then the mouse will be inoculated with more antigen. Once the titer is high enough in the serum, cell fusion is performed. The mice are injected with one final antigen dose three days before cell fusion takes place, but at least two weeks after they received their previous immunization. The mice are euthanized and their spleen or lymph nodes are removed to produce hybidomas. Step 3: preparation of myeloma cells for fusion with the antibody-producing cells from the mice. The antibody-producing cells from the mice have a limited lifetime whereas the myeloma cells are immortal. When the two cells are combined, they result in cells that are capable of immortal growth and continued production of antibody. The myeloma cells are cultured with 8-azaguanine so that they will not die when exposed to hypoxanthine-aminopterin-thymidine (HAT), which is used to positively select for only antibody producing cells that have fused with the myeloma. The myeloma cells also have been modified to lack hypoxanthineguanine phosphoribosyl transferase (HGPRT-), this means that the myeloma cells cannot synthesize purines and in order to survive they need to fuse with the normal GHPRT ${ }^{+}$mouse cells. Step 4: Fusion of the myeloma and antibodyproducing cells. Fusion between the antibody-producing cells and the myeloma cells is achieved by co-centrifuging them at a ratio of $10: 1$ in polyethylene glycol causing cell membranes to fuse. Since the fusion is random, only some of the 
fused cells are between the antibody-producing cells and myeloma cells.

Selection for these cells uses HAT media. The aminopterin in the HAT is a folic acid analogue that blocks the biosynthesis of purines and pyrimidines, ceasing DNA synthesis. Any antibody-producing/antibody-producing fused cells will not be able to survive in the HAT media because they cannot synthesize DNA. Any myeloma/myeloma-fused cells will die in a few weeks because they lack HGPRT. The antibody-producing/myeloma fused cells will have HGPRT provided by the antibody-producing cells and the myeloma cells will keep them alive in the HAT solution. 500 hybrids that are usually made per mouse spleen and only around 20 to 30 of those are usable hybrids. The antibody-producing/myeloma fused cells are placed into well plates that contain feeder cells, believed to promote the growth of hybridoma cells through supplying growth factors. Step 5: cloning the hybridoma cell lines. The hybridoma cells from the well plates are grown in tissue culture and then selected for based on antigen binding.

After mAbs are developed, they can be produced in vitro using batch tissue-culture or semipermeable-membrane-based system production if the specific cell line allows in vitro production. Batch tissue-culture is used to grow hybridoma cultures in batches from which pure mAb can be extracted. Fetal bovine serum commonly used as the tissue-culture media however serum-free media has been developed to avoid possible contamination. Batch tissue-culture methods are low cost, easy to perform, and only take around 3 weeks to produce the same quality of mAb as those produced in vivo. However, batch tissueculture does not produce high concentrations of mAb, usually below $20 \mathrm{ug} / \mathrm{ml}$. 
While it easy to perform this method, large amounts must be produced to obtain usable amounts of antibody. Also, some antibodies denature when they are purified and concentrated from the culture during batch tissue-culture. Another option for in vitro production of antibodies is semipermeable-membrane-based system production. A barrier is used that has a low-molecular-weight cutoff allowing for cells to grow at high densities; when hybridoma cells grow at higher densities in culture the result is the production of higher amounts of harvestable mAb. Semipermeable-membrane based systems make it possible to isolate the cells and mAb in the chamber separated from the culture media. This allows for supplementation of growth factors to optimize the growth of the hybridomas and keeps nutrients for the cells and any undesired cell waste away from the mAb product through diffusion. Media can be constantly replaced without losing any cells or mAb contained in the small chamber. Semipermeable-membrane based systems allow for higher concentrations of mAb to be produced in low volumes of culture, however, the mAb may be contaminated by any dead cell products that do not diffuse through the membrane. Semipermeable-membrane-based systems are more technically difficult to run than batch tissue-culture methods. These two techniques allow for in vitro options that do not involve euthanizing animals while still producing functional monoclonal antibodies. The production of monoclonal antibodies is necessary for the development and production of diagnostic and therapeutic techniques [4]. 


\section{Polyclonal antibodies}

Polyclonal antibodies differ from monoclonal antibodies by recognizing multiple binding sites for one antigen, meaning that there is a heterogeneous mixture of antibodies all with different affinities for the antigen within one sample. This causes polyclonal antibodies to be prone to variability between batches, unlike monoclonal, which are identical between batches. One reason that polyclonal antibodies can be more desirable than monoclonal antibodies is because monoclonal antibodies may be too specific to detect an antigen if any mutation has occurred [5]. Polyclonal antibody production is similar to monoclonal antibody production in that a laboratory animal is used, however, various animals may be selected depending on the amount of serum needed and how easily it can be obtained. Some animal choices include mice, chickens, guinea pigs, goats, and sheep. Antigen is injected subcutaneously, intramuscularly, intradermaly, or intraperitonealy in several sites on the animal along with an optional adjuvant, to promote the immune response at the injection site. Blood is taken from the animal, up to $10 \%$ of the blood volume of the animal at a time every 3 to 4 weeks from which the antibody is harvested [6]. The blood is centrifuged to separate the red blood cells from the serum. An antigen-bound sepharose bead column can be used to separate the desired antibodies from the other antibodies in the serum solution. The serum is run through the column and only the desired antibodies will bind to the antigen-bound beads and all the others will run through. The bound antibodies are then eluted from the sepharose beads and collected as polyclonal antibodies that all bind to the same antigen. 


\section{Use in research}

Antibodies are used for many different applications including diagnostic analysis and disease targeting. Antibodies can be used as drugs with specific targeting; monoclonal antibodies are used to make cancer cells more visible to the immune system by attaching to parts of the cancer cells and alerting the immune system to attack it. Antibodies can also be used to block growth signals, stop new blood vessels formation, or deliver radiation to specific cancer cells [8].

One of the most common techniques involving antibodies is ELISA, which is used for determining the presence or absence of diseases such as HIV. ELISA (enzyme-linked immunosorbent assay) can be performed several ways, all involving the use of antibodies. Firstly, indirect ELISA is performed when the target antigen is adsorbed to the bottom of the wells on a plate and antibodies extracted from a patient are allowed to interact with it. Finally anti-human antibodies are used to image the plate to determine if there was binding between the patient antibodies and the antigen. If binding occurs then the individual has that antigen present in body. Indirect ELISA is performed to determine whether an antigen is present or not, the second ELISA technique allows for detection and quantification of the antigen. The second most common form of ELISA is sandwich ELISA. Sandwich ELISA is performed by adsorbing anti-antigen antibodies to the bottom of the wells on a plate. The sample from the patient is then added to the wells to allow interaction with the antibody. Next, another antibody is added that will also bind to the antigen. Finally an enzyme linked antibody is added that will interact with the second antibody, the enzyme will 
produce a detectable signal that will allow for the quantification of antigen present [9].

Western blot also utilizes antibodies; specific proteins may be identified within the gel through modified antibodies that allow for imaging. Proteins are separated by size using SDS-PAGE and then electrophoresis is used to transfer them to nitrocellulose paper. A process similar to that in ELISA is used where the proteins are incubated with primary antibody and then secondary antibodies that allows for visualization and quantification of the amount of protein.

A relatively new technique for antibody use are microarrays where anywhere between 5,000-10,000 proteins can be analyzed at a time. Proteins are captured on the chip by specific primary antibodies and then analyzed using secondary imaging antibodies. These imaging antibodies are often recombinant antibodies that allow for detection by fluorescence, nano-electrodes, and surface plasmon resonance [10]. This technique is most commonly used for overall proteomic analysis.

Antibodies are also used for in-field testing that has the advantage of being quick, easy to perform, with easily interpreted results. One such test is the pregnancy blood test. hCG is present in the blood of pregnant women, an immobilized primary capture antibody is used to bind to this molecule and a secondary signal antibody is then used to give a response. One important thing to note for test like this is the possibility of false positives due to interfering antibody interaction. This interaction occurs if the Fab regions on the interfering antibody interact with those on the primary and secondary antibodies. This will 
cause the signaling antibody to indicate a positive, even though no antigen is present. Another diagnostic test that has been developed using antibodies are blood typing kits. Antibodies are immobilized to the surface of the testing kit, when blood interacts with the antibodies it will coagulate if antigen is present. Based on which antibodies the blood coagulates with both the blood type and the Rhesus factor can be determined.

As more selective and stable binding between antibodies and antigen are required, new antibodies modifications are being developed in the laboratory to meet these needs. The Fab segments of antibodies have been mutated in order to manufacture antibodies with higher affinity binding. This is achieved by improving the contact and molecular interactions between the antibody and the antigen [11].

\section{Advantages/disadvantages}

There are many different reasons for selecting between monoclonal and polyclonal antibodies when performing an experiment. Polyclonal antibodies are selected for use when an increased signal is desired since there are various antibodies that all bind to the same antigen at different sites. Polyclonal antibodies are also less sensitive towards changes in the antigen allowing for detection of any mutated antigen. Monoclonal antibodies, on the other hand, are selected when highly specific binding is needed between antibody and antigen. Since mAbs are produced by hybridomas more quantity can be produced of identical and pure antibodies. However, polyclonal antibodies are much less expensive to produce than monoclonal antibodies and require a lower level of 
technology and skill. Polyclonal antibodies also take significantly less time to produce than monoclonal antibodies do.

There are also advantages and disadvantages to utilizing antibodies instead of other molecules. Antibodies cannot easily be produced for toxins due to the fact that they are produced in animals that cannot tolerate them long enough to produce an antibody for them. Another restriction of antibodies since they are produced to function in cells is that for diagnostic techniques, antibodies are often not able to function in non-physiological conditions. Since antibodies have complex, protein based structures they are denatured easily due to temperature, susceptible to oxidation or deamidation, degradation or aggregation, leading to a limited shelf life.

\section{FUNDAMENTALS OF APTAMERS}

\section{Production}

Aptamers are produced through a method called the SELEX (systematic evolution of ligands by exponential enrichment) process. This process begins with a random sequence oligonucleotide library that is obtained by combining the four nucleotide building blocks, adenine, thymine, guanine, and cytosine for DNA and adenine, cytosine, guanine, and uracil for RNA. Depending on if a RNA or DNA aptamer is being created, the specific four nucleic acids molecules are added together in a concentration based on the coupling efficiency of each individual monomer. A fixed sequence is used as a primer for binding amplification of the oligonucleotide sequences. The length of nucleotide positions in the random region determines how complex the oligonucleotide library will be; 
for example, if a library has a "40-nucleotide random region" the library will contain up to $1.2 \times 10^{24}$ different sequences. However, a library is typically limited by chemical synthesis to only contain around $10^{14}-10^{15}$ sequences [12].

Once a random library is synthesized, it is exposed to the target of interest for which the aptamer is being designed. The target of interest is bound to nitrocellulose paper prior to being exposed to the oligonucleotide sequences so that all non-binding sequences can then be washed off, leaving the bound sequences to attached to the target on the nitrocellulose paper. Once the unbound sequences are washed off, the bound sequences are eluted and amplified using PCR for creating a DNA aptamer or RT-PCR for an RNA aptamer. The binding and amplification process is repeated in order to increase the affinity for the bound molecules. Typically around 8-15 cycles of affinity enrichment are performed, taking around 2-3 months to produce high affinity binding aptamers. The SELEX process creates aptamers that over $90 \%$ of the time are able to bind to the target and are around $70-80$ nucleotides in length [12]. These sequences can be paired down from the 70-80 nucleotide length to cut out any areas that do not play an important role in the three dimensional structure of the aptamer for binding with the target molecule.

After aptamers are fully developed through the SELEX process, they can be produced in less than 3 days, resulting in identical product each time. Another step may be added to the SELEX process resulting in counter-SELEX where aptamers can be counter selected for. This comes in useful when creating an aptamer that will bind to a cell, but only those that display a particular 
characteristic, such as being infected with cancer. Counter-SELEX is accomplished by eliminating any oligonucleotides that have affinity for motifs present on non-targeted cells.

\section{Structure}

Aptamers are composed of either ssDNA or RNA that are usually $70-80$ nucleotides in length. The aptamer folds into a three dimensional structure that will interact with the antigen of choice, much like an antibody does. The molecule that interacts with the aptamer is called a ligand and aptamers are designed to specifically bind to one and only one ligand.

RNA structure is determined by the primary, secondary, and tertiary interaction that it undergoes. Primary structure is determined by the nucleotide sequence that makes up the RNA strand. The secondary structure of the RNA is determined by the two-dimensional interactions between the individual nucleotides that make up the RNA. For RNA common secondary structures are duplexes, single-stranded regions, hairpins, bulges, internal loops, and junctions (Figure 2) [13]. The tertiary structure of RNA is determined by the interactions between the different regions of the two-dimensional secondary structures of the RNA strand that make up the three-dimensional shape of the molecule. Some examples of common

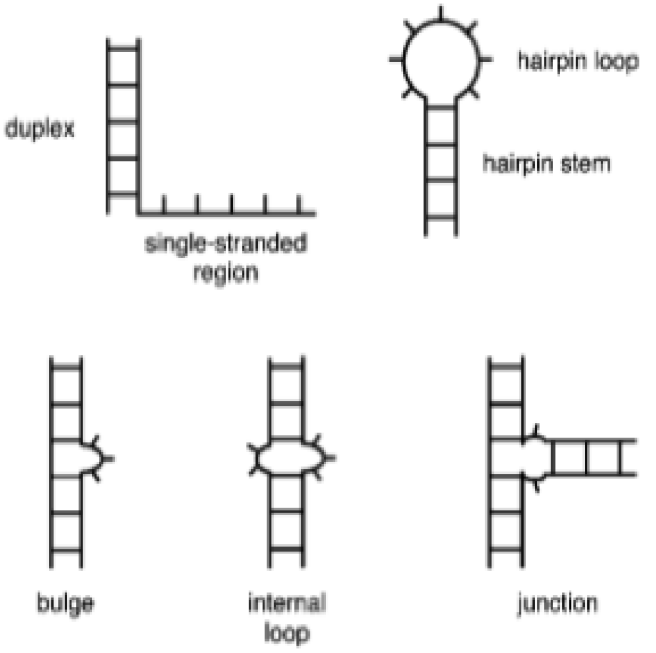

Figure 2: RNA Secondary Structures Some of the common two-dimensional secondary structures of RNA. (Figure from Batey, Robert T. et al.) 
tertiary interactions are triple interactions: the RNA single strands interact with double-stranded RNA complexes through hydrogen bonding, pseudoknots: form where the RNA nucleotides in a hairpin loop pairs with a single stranded region of RNA, kissing hairpins: formed when unpaired nucleotides from one hairpin loop interacts with another unpaired nucleotide in another loop [14].

The structure of RNA allows for reversible denaturation, meaning that the tertiary and secondary structures are no longer formed when the RNA molecule is exposed to an atmosphere that facilitates denaturation. Once the denaturing climate is removed, the RNA will fold back into its original form with the secondary and tertiary structures (Figure 3). One issue with RNA aptamers in

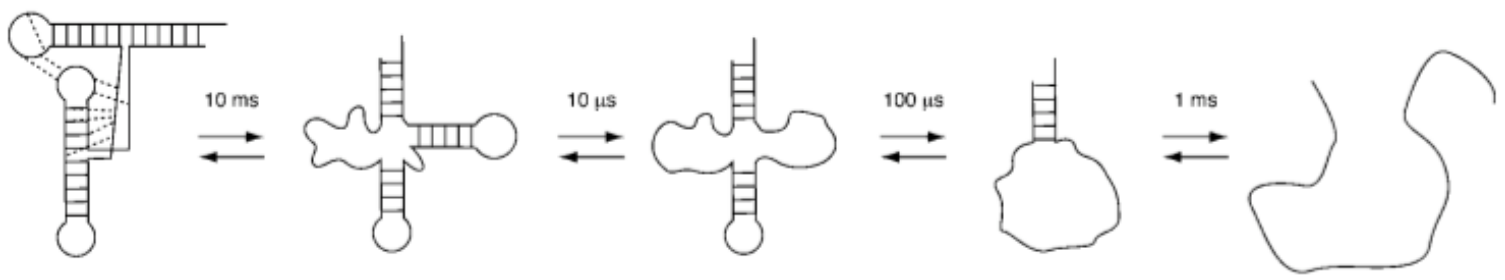

Figure 3: Reversible denaturation pathway of RNA

On the far right the RNA is completely denatured, slowly the secondary structures resulting from the interactions between the nucleotides are achieved. Finally on the far left the tertiary structures are completed, resulting in the three-dimensional shape of the RNA. (Figure from Batey, Robert T. et al.)

regard to their structure is the ability of nucleases to degrade the RNA; therefore modifications have been developed to protect the RNA aptamers. Modifications have be made to the 2' position on the pyrimidine nucleotides by adding $-\mathrm{F}$ or $\mathrm{NH}_{2}$ functional groups to prevent denaturation by nucleases.

Another solution to the degradation of RNA aptamers is to use a single stranded DNA aptamer instead. Single stranded DNA (ssDNA) acts much like RNA in that it is able to interact with itself to form into two and three-dimensional shapes through interactions. ssDNA will form secondary structures just like the 
RNA aptamers: internal loops, hairpin loops, bulges, dangling ends, terminal mismatches, coaxial stacking, and multibranched loops [15]. It has been suggested that SsDNA tertiary structure is based on the guanine quadriplex structure, which is part of the secondary aptamer structure and is made up of one, two, or four oligonucleotide strands [16]. DNA aptamers are also suspected to have a partially disordered tertiary structure when no antigen is bound [17]. Furthermore, in a thrombin aptamer study, it was indicated that due to the fact that the tertiary structure of aptamers is highly specific, is very important for the binding interaction with the ligand [18].

Modifications are often made to the structures of both RNA and ssDNA aptamers in order to increase their usefulness in laboratory techniques or to increase stability. The increase in RNA stability has already been discussed; this is achieved through producing the aptamers from libraries that contain the modified pyridinines instead of normal ones. Other modifications that have been developed are made at the $\mathrm{C}-5$ position on the pyrimidines and include the addition of $-\mathrm{I}$ or $-\mathrm{Br}$ for photo or chemical crosslinking, $-\mathrm{SH}$ for chemical crosslinking, and amino acid side chains for chemical dressing [12]. 3'-end caps have also been added to aptamers in order to reverse their polarity, and as such preventing any 3 ' exonuclease activity. PEG is also added to the 5' end of aptamers to extend the in vivo half-life of aptamers [19]. One interesting result of modification is that the primary and secondary structures of the aptamers are significantly changed from those produced without the modifications. 


\section{Use in Research}

The structure of aptamers allows them to bind to target molecules or cells with high affinity and specificity. Aptamers have so far been developed for targets such as metal ions, organic dyes, drugs, amino acids, co-factors, aminoglycosides, antibiotics, nucleotides, and peptides [12]. One specific use for aptamers is their utilization for lead detection in the environment; gold nanoparticles are coated with engineered molecules made up of a 12-mer aptamer, a DNAzyme, and a substrate. In the absence of lead, the substrate hybridizes with the aptamer causing the gold to aggregate, resulting in a blue color. If lead is present, then the DNAsyme catalyzes hydrolytic cleavage of the substrate so that the aptamer is no longer able to bind. This prevents the aggregation of the gold and produces a red color. UV-vis spectroscopy is used to detect these color changes as well as to quantify the amount of $\mathrm{Pb}^{2+}$ present in the sample [20].

Aptamers have also been modified using Photo-SELEX to create aptamers that are capable of simultaneous quantification. During the SELEX process modified nucleotides are used that can be activated by the absorption of light. Aptamers made up of these nucleotides covalently bond with their target when activated by light, leading to greater sensitivity and specificity. Chips have been made using Photo-SELEX-evolved aptamers that are incubated with extremely dilute sample and can allow for imaging and quantification of the sample [21]. 
Counter-SELEX has already been used to develop an aptamer against glioblastomas, one of the most common brain cancers. Aptamers such as this can be used as molecular probes for the detection, characterization, and isolation of tumor cells when they have been modified with fluorescent labels. Aptamers have successfully been used to target and identify cells within bone-marrow aspirates [22].

Aptamers have also been developed as drugs that target specific molecules. In 2004, Macugen was developed and approved by the FDA as a drug against age-related macular degeneration. As of 2012, Macugen is still the only aptamer based therapeutic that has been approved for market by the FDA. Macugen is an RNA aptamer the binds to all isoforms of VEGF. There have been other attempts to develop antigen-based drugs such as an antidotecontrolled anticoagulant RNA aptamer. This aptamer drug functions by using an aptamer that elicits an anticoagulant effect by binding and inhibiting the activity of coagulation factors. A complimentary aptamer region was also developed that upon injection binds and neutralizes the active form of the drug. The drug went to phase 1 of clinical trials but the study was terminated because the protocol defined safe threshold was exceeded in one subject who received the highest dosage [23].

Aptamers have also been used in techniques such as flow cytometry and immunosensors, taking the place antibodies. Aptamers are showing promise to be used as sensors based on electrochemical detection; since aptamers are polyanionic they can be used to sense changes in conduction based on the 
presence or absence of a target molecule. One study used this technique to develop a microfluidic electrochemical aptameric assay integrated on a chip that was able to detect selected targets in a mixed sample with no interference [24].

Other areas of research where aptamers have begun to take hold are as molecular switches, for detection of proteins (being referred to as "Eastern blotting" completing the blotting technique quadruple), aptamer arrays for proteomic analysis, and many other clinical applications for disease drug analysis.

\section{Advantages/disadvantages}

Aptamers offer an alternative to traditional molecular choices such as antibodies. Aptamers offer an advantage over antibodies because they can be created for any molecule, even those that are toxic to biological systems. Aptamers also allow for a plethora of modification options, from making them more detectable to changing the kinetic on- and off-rates, something much more difficult to do with antibodies [12]. Aptamers offer high reproducibility, meaning that there is little to no batch-to-batch variability. Due to the nature of RNA and ssDNA, aptamers are able to undergo reversible denaturation. This reversible denaturation holds one of the highest advantages of aptamers over antibodies as it offers for production of reusable diagnostic testing platforms, further lowering the cost of production. Products such as this would be ideal for in field-testing; especially in third world countries where clinical analysis is difficult and cost is a huge factor. Aptamers are also much more cost effective to produce than 
monoclonal antibodies are, taking around the same amount of time with little to no hands-on interactions needed and less experienced lab technicians required.

So far one of the only major limitations that has become apparent for aptamers is the vulnerability of RNA aptamers, but the aptamer may be modified as talked about previously to compensate for this. SELEX can also be laborious to carry out, however, automated systems now exist that are faster and more economical that require little to no human intervention that can run multiple SELEX experiments at a time.

Another current disadvantage to the use of aptamers is the fact that their invention was still less than 20 years ago so many of the applications and production techniques related to aptamers are still protected under patents. Gilead Science acquired many of the patents that are related to the SELEX process as well as the actual aptamer structures that have been produced [25]. The length of patent protection is 20 years after filing takes place so the first of their patents, filed in 1992 will expire this year along with several other patents in the coming years. Archemix is another company that has intellectual property rights to aptamers, specifically to the exclusivity to use of them in therapeutic applications [25]. The first of their patents was filed in 2002 so their patents will not begin to expire until 2022. However, they have licensed out the rights of their patents to several other companies to use in the research and develop other therapeutic applications. SomaLogic has exclusivity to the use of aptamers for diagnostic applications [25], with their first patent filed in 1999 and expiring in 2019. Since many of the patents initially filed that relate to aptamers are starting 
to expire, there is a high likelihood that new applications for aptamers will continue to be developed in upcoming years that involve previously patent protected techniques.

An alternative to both aptamers and antibodies that are also being researched are molecular imprinted polymers (MIP). A MIP is a polymer that is made using a three dimensional template that is then removed, leaving behind a three-dimensional polymer with complimentary spaces from where the template was removed. Unlike both aptamers and antibodies, MIPs are made of entirely synthetic products making them even more stable than aptamers are with the ability to withstand high temperatures, $\mathrm{pH}$ ranges, and long-term storage. MIPs have the ability to recognize and bind to specific target molecules and offer another option besides antibodies and aptamers that have to for use in many techniques that currently employ antibodies [25].

\section{Fundamentals of Surface Plasmon Resonance (SPR)}

\section{Surface Plasmon Resonance History and Theory}

R.W. Wood first discovered the theory behind surface plasmon resonance in the early twentieth century with his work on diffraction on diffraction gratings due to the excitement of surface plasmon waves. Kretschmann and Otto continued to research surface plasmons through optical excitation in the 1960s.

Since 1992 the amount of research publications that mention the use of SPR has exponentially grown each year from only a handful in 1992 to over 500 in the first half of 2006 [26]. 
Surface resonance is a charge-density oscillation that occurs at the interface of two media that have opposing dielectric constants [27]. This oscillation results in a surface plasmon wave. Surface plasmon waves are TMpolarized waves, meaning that the magnetic vector is perpendicular to the direction of the propagation and parallel to the plane of the interface. Surface plasmons are the collective oscillations made by the free electrons in a metallic film. When exposed to specific conditions, the surface bound plasmons will resonate with light, resulting in the absorption of the light. These conditions are extremely sensitive with respect to the refractive index of the medium that is located adjacent to the metallic film, which means that the presence of any molecules on or near the surface of the film can easily be detected.

One common method used to excite the surface plasmons into their resonance oscillations is the Kretschmann configuration that uses a p-polarized laser and a metal film, typically gold or silver. The incident angle reflection of the laser on the metal film is used to achieve the resonance of the surface plasmons. When the incident angle is small and resonance is occurring, the incident light may be absorbed by the metal film resulting in a low intensity of reflection. This means that the reflected light remains at a high intensity for a large range of angles except at the resonance angle.

$$
\sin \left(\theta_{R}\right)=\sqrt{\frac{\varepsilon_{1} \varepsilon_{m}}{\left(\varepsilon_{1}+\varepsilon_{m}\right) \varepsilon_{2}}}
$$

In order to find the resonance angle, $\theta_{\mathrm{R}}$, Equation 1 is used. $\varepsilon_{1}$ can be water, air, or a variety of other solvents or

Equation 1: SPR resonance angle Used to calculate the resonance angle, $\theta_{R}$. $\varepsilon_{2}$ is the dielectric constant of the prism, $\varepsilon_{m}$ is the real part of the dielectric constant of the metal film, $\varepsilon_{1}$ is the dielectric constant of the testing medium to which the metal film faces. 
solutions, often for biological SPR experiments PBS buffer is used. A shift in $\theta_{R}$ occurs then there are molecules near or on the surface of the metal film. The shift in the resonance angle is proportional to the amount of molecules that are adsorbed on the metal film surface, through observing the changes in the resonance angle it is possible to measure the association, disassociation, and affinity binding constants for the molecules [27].

\section{SPR Components and System}

BIAcore AB released the first commercially available SPR system in 1990 and since then several other companies have also developed and released commercial SPR systems. Each SPR machines, no matter which company produced it, has the same basic components and systems as they all perform analysis using the same basic theory.

The main difference between SPR systems is how automated they are and how many analysis channel

exist; these two components both directly relate to the cost of the SPR system. The main components in all systems are the flow cell through which the sample and running buffer flow.

There is a prism (Figure 4) on which the metal film chip is placed, often with a specific

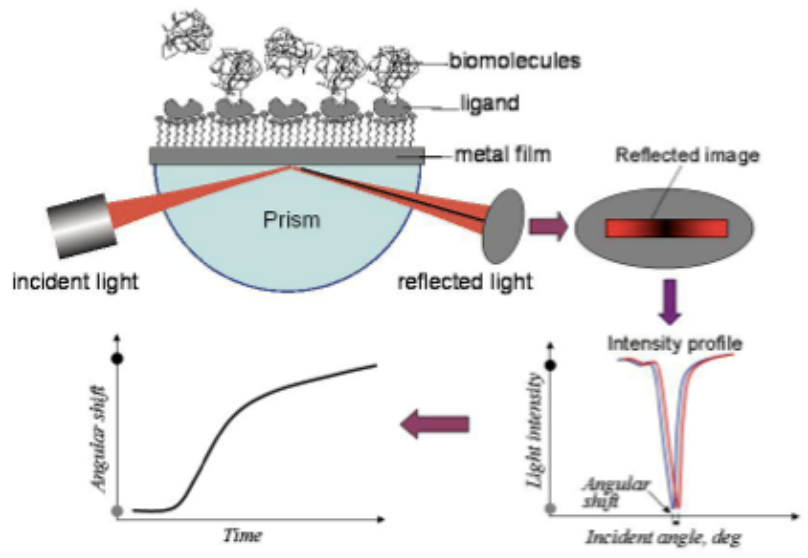

Figure 4: Surface Plasmon Resonance Shows the interaction of the molecules and their effect on the metal film surface and the resulting shift in reflected light from the laser. This shift is translated to graphical data allowing for analysis of the kinetic interactions on the molecules. (Figure from B!-2000 SPR Manual v5.4) 
molecule, the ligand, immobilized on the surface. The binding or interacting molecule to the ligand is injected into the buffer solution as it flows across the surface of the functionalized metal chip surface. As the ligand and its conjugate interact the refractive index will change, this change is measured and imputed into the system's software that analyses it and shows the mDeg change in the angle of refraction. This information can be further analyzed to give the kinetic data for the interaction between the ligand and the interacting molecule [29].

Due to the sensitivity of SPR, all liquids that are run though the system must be either sonicated or degassed to remove any dissolved gas molecules in the solution. When injecting samples into the machine care must be taken to avoid gas bubbles in the injection syringe as well. Dissolved gasses in the solution will result in random spikes in the sensorgram that will interfere with the results. Care must also be taken when cleaning and preparing the prism surface before placing the chip on it. If the prism is scratched at all, the refraction angle will not be able to properly observe molecular interactions taking place. The flow rate of the buffer should not exceed $150 \mathrm{uL} / \mathrm{min}$ or there is not enough time for the kinetic or equilibrium constants to be extracted from the flow data [28]. The flow rate cannot be too slow either or mass transport limited kinetics will occur [28].

\section{Use in research}

SPR has been used in several areas of research including for the measurement of physical quantities of materials, for chemical sensing analysis, and for biosensing analysis. SPR was first used in 1983 for biosensing analysis, and in 1994 the first real-time biospecific interaction analysis was developed [27]. 
SPR provides detection and analysis for chemical, biochemical, and biological substances in the areas of medicine, environmental analysis, and drug and food safety. One advantage of using SPR is that it is a label-free process that still allows for the analysis between molecules. Another huge benefit of using SPR in analysis is that only a very small amount of sample is required and machines offer real-time analysis and the use of multi-channels for the analysis of simultaneous samples.

SPR has been utilized to perform microscopy as SPRM or SPR imagine (SPRI). SPRI allows for the measurement of DNA and RNA binding to DNA arrays or DNA-binding proteins to dsDNA arrays, proteins to protein and peptide arrays, and ligands to protein arrays. SPRI can determine the on- and off-rates and the equilibrium binding constants for all of these interactions simultaneously, sometimes on the order of more than 1,000 at a time. This technique offers cost and time efficient detection and analysis technique [30]. SPRI is accomplished by spotting probe molecules onto the analysis surface, this must be done in a way that will not denature or inactivate the probe, will enable the probe to survive washing for multiple use, and makes the probe available for other molecules to interact and bind to. There are many different methods and materials that have been developed to functionalize the surface of SPR chips.

The first method that is commonly used to attach molecules to the surface of SPR chips is through thiol-linking the molecule directly onto the gold metal film. Attachment of molecules to the gold surface through thol-linking is achieved by exposing the gold surface to thiol-alkane linked molecules and allowing 
chemisorption to occur; this binding is vey stable and allows for complex media buffers to be used [31]. Another common immobilization method is achieved by using carboxymethyl dextran (CM-Dextran) that forms a self-assembled monolayer (SAM) onto which other molecules can easily be bound. An activating kit is used to activate the dextran surface and allows for amine coupling occurring between the dextran particles and the molecules will become immobilized on the surface. Other chemical attachments and surface modifications that are used include aldehyde coupling, streptavidin-biotin linking, silicate and silicon functionalized surfaces, and biocoatings [32].

SPR has become a common laboratory technique to observe the binding kinetics between modified antibodies and aptamers and the ligands they were created to bind to. The VEGF-binding affinities for the Fab fragments of antibodies were observed in an experiment that was attempting to select for optimized binding in a modified anti-VEGF antibody [33]. Similarly SPR was used when screening for an improved anti-VEGF DNA aptamer. Using SPR not only allowed for kinetic analysis of the binding between the aptamer and VEGF, but it was also used to observe the role that $\mathrm{KCl}$ played in the three-dimensional shape of the aptamer [34]. 


\section{MATERIALS AND METHODS \\ SPR CALIBRATION AND TECHNIQUE}

The SPR instrument must be calibrated every couple months in order to align the signals given by each microfluidic channel so that they are identical. This calibration was done using a $1 \%$ ethanol solution and by comparing the measured $\mathrm{mDeg}$ response

in each channel to the New Calibration Value $=$ Old Calibration Value $\times \frac{\text { Expected }}{\text { Measured }}$ expected response Equation 2: SPR calibration Used to calculate the new values to calibrate the channels in the SPR machine.

changes using Equation 2.

Gloves were worn for the entire experiment to avoid contamination of the machine and exposure to the solutions. The $1 \%$ ethanol solution was made by adding $0.1 \mathrm{~mL}$ of $99 \% \mathrm{EtOH}$ to $10 \mathrm{~mL}$ of $\mathrm{DI} \mathrm{H}_{2} \mathrm{O}$, both the $\mathrm{EtOH}$ and $\mathrm{DI} \mathrm{H}_{2} \mathrm{O}$ were previously degassed for an hour in a bell jar apparatus to remove any dissolved gases in the solution. The SPR machine was then prepared for use. Two $10 \mathrm{~mL}$ syringes were filled with the $\mathrm{DI} \mathrm{H}_{2} \mathrm{O}$ as running buffer and placed in the Syringe Cradle and fastened with the Retainer Bar. The Drive Bar was adjusted to just barely touch the end of both syringes. Finally the syringes were screwed into the flow tubing.

Next the prism surface was prepared for a new chip. There was an old chip still on the prism surface that was maneuvered using a cotton swap so that the prism was not scratched and then tweezers were used to fully remove the chip. To clean the prism surface $99 \% \mathrm{EtOH}$ and cotton swabs were used. The cotton swab was soaked in $\mathrm{EtOH}$ and then moved in a spiral pattern around the 
prism surface starting in the middle and working outwards. This process was repeated until the prism surface was completely clean. The Flow Cell channels were also cleaned using EtOH and cotton swabs. Mounting the SPR chips on the prism was achieved by placing a small drop of index matching oil in the center of the prism. The drop should be only 3-5uL or it can leak out from under the chip and contaminate the experimental results. To ensure the drop was the right size, the initial few drops of oil were dabbed off onto the rim in the bottle and then the glass transfer rod was lightly placed above the prism until as small drop was left on the prism. The chip was inspected to determine which side was gold and which side was glass, this was achieved by lightly scratching the edge of both sides of the chip. The gold side of chip showed scratch marks and the glass side did not, the chip was then placed on top of the prism. To ensure no gas bubbles were trapped between the chip and the prism, one side of the chip was placed down before the other using tweezers. A cotton swab was then used to move the chip around slightly. The Flow Cell was then moved into place on top of the chip. The machines were then turned on and the flow rate of the running buffer was set to $150 \mathrm{uL} / \mathrm{min}$ to force out any trapped air bubble in the tubing. After $\sim 5$ minutes the flow rate was reduced to $50 \mathrm{uL} / \mathrm{min}$ and the laser was turned on. To make sure that there were no air bubbles in the flow cell there is a viewing window that should show two similar SPR dark lines about $4 \mathrm{~mm}$ wide each. This was observed and the machine was allowed to warm up for around 30 minutes, until signals in both channel 1 and channel 2 were flat. 
The calibration was then performed. The machine was placed into serial mode. The default calibration value is 3,000 ; this value was used as an initial calibration to run the first $1 \%$ ethanol injection. The expected mDeg result was supposed to be 60 . The injection was made using a $200 \mathrm{uL}$ syringe and injecting around 150uL of sample into the Injection Valve. The valve was then turned to the 'Injection' position until the countdown on the computer reached 15 seconds left out of 96 , when it was switched back to 'Load.' After each injection of $1 \%$ ethanol Equation 2 and the mDeg measuring software was used to find the adjusted calibration value for each channel until they both were $60 \mathrm{mDeg}$.

In order to get a feel using the instrument and the software an additional ethanol dilution experiment was performed after the machine was calibrated. Two additional solutions of ethanol were made along with the $1 \%$ ethanol solution: a $0.25 \%$ solution and a $0.0625 \%$ solution. The $0.25 \%$ solution was made by pipetting $1 \mathrm{~mL}$ of the $1 \%$ ethanol solution into $3.01 \mathrm{~mL}$ of $\mathrm{DI} \mathrm{H} \mathrm{H}_{2} \mathrm{O}$ and the $0.0625 \%$ solution was made by pipetting $1 \mathrm{~mL}$ of the $0.25 \%$ solution into $3.0025 \mathrm{~mL}$ of DI $\mathrm{H}_{2} 0$. These solutions were injected into the machine the same way as the $1 \%$ ethanol solution was and the results were saved so that they could be analyzed using the kinetic software.

\section{Selection of Carboxymethyl Dextran}

Carboxymethyl Dextran (CM-Dextran) is a polyanionic derivative of Dextran. Dextran is composed of $\alpha(1 \square 6)$ linked glycan that has side chains attached to the 3-positions of the backbone glucose units [35]. CM-Dextran allows for molecules to be immobilized on its surface though amine coupling. The 
CM-Dextran chips were ordered from Biosensing Instrument and stored at $-20^{\circ} \mathrm{C}$. Activation kits for the CM-Dextran Sensor Chips were also ordered from Biosensing Instrument and stored at $-20^{\circ} \mathrm{C}$ as well.

\section{USE OF VEGF}

VEGF-A is a member of the PDGF/VEGF growth factor family and codes or a protein that acts on endothelial cells with various effects. Some of these effects include: increased vascular permeability, induction of angiogenesis, vasculargenesis, promotion of cell migration, and inhibition of apoptosis [36].

VEGF-A was one of the molecules for which both aptamers and antibodies have been developed, making it a perfect molecule for comparing the kinetic interactions between it and both its aptamer and antibody conjugates. The VEGF-A, also known as VEGF 165, was ordered from Syd Labs as recombinant human VEGF 165. This molecule was produced in HEK 293 derived cells and had a molecular weight of $19.2 \mathrm{kDa}$. The stability of the VEGF-A was for 12 months if stored as $-20^{\circ} \mathrm{C}$ to $-70^{\circ} \mathrm{C}$ in evaporated form, 1 month after reconstitution if stored at $2^{\circ} \mathrm{C}$ to $8^{\circ} \mathrm{C}$ in sterile conditions and for 3 months after reconstitution of stored at $-20^{\circ} \mathrm{C}$ to $-70^{\circ} \mathrm{C}$ under sterile conditions [37].

The VEGF was diluted to a $25 \mathrm{nM}$ solution, a $50 \mathrm{nM}$ solution, and a $150 \mathrm{nM}$ solution. $10.417 \mathrm{~mL}$ of $\mathrm{DI} \mathrm{H}_{2} \mathrm{O}$ was added to the 10ugram of VEGF sample to create a $50 \mathrm{nM}$ stock solution. The $50 \mathrm{nM}$ solution was then diluted to half by adding $1 \mathrm{M}$ PBS buffer to create the $25 \mathrm{nM}$ solution. The $150 \mathrm{nM}$ solution was produced by adding $2 \mathrm{uL}$ of $1.5 \mathrm{mg} / \mathrm{mL}$ VEGF solution to $1037.67 \mathrm{uL}$ of $1 \mathrm{M}$ PBS buffer. 


\section{IMMOBILIZATION OF VEGF AND ANTIBODY/APTAMER INTERACTIONS}

VEGF-A was immobilized to the CM-Dextran surface in order to observe the kinetic interactions between it and the anti-VEGF aptamer and anti-VEGF antibody. The solutions were made at a concentration of between 25 and 100nM due to the fact that the SPR machine is extremely sensitive to samples and only a small amount is required. Typically concentrations used for immobilization are between 10-100nM.

\section{Antibody Solution}

The anti-VEGF-A antibody was ordered from Bioss and was a rabbit antiVEGF-A polyclonal unconjugated antibody. The antibody was stored at $-20^{\circ} \mathrm{C}$, a temperature where it would be stable for at least one year. The anti-VEGF-A antibody solution was prepared in three concentrations: $100 \mathrm{nM}, 50 \mathrm{nM}$, and $25 \mathrm{nM}$. The $100 \mathrm{nM}$ solution was made by pipetting $2222.22 \mathrm{uL}$ of $\mathrm{DI} \mathrm{H}_{2} \mathrm{O}$ with 100ugram of the anti-VEGF antibody. The 50nM concentration was made by pipetting $1 \mathrm{~mL}$ of the $100 \mathrm{nM}$ solution into $1.5 \mathrm{~mL}$ of $\mathrm{DI} \mathrm{H} \mathrm{H}_{2} \mathrm{O}$. The $25 \mathrm{nM}$ solution was made by pipetting $1 \mathrm{~mL}$ of the $100 \mathrm{nM}$ solution into $3 \mathrm{~mL}$ of $\mathrm{DI} \mathrm{H}_{2} \mathrm{O}$. Once reconstituted, the antibody was stored at $2-4^{\circ} \mathrm{C}$, a temperature where it was stable for at least two weeks [38].

\section{Aptamer Solution}

The anti-VEGF-A aptamer sequence was taken from Nonaka et al. from when they searched for an improved anti-VEGF-A DNA aptamer. The sequence of the aptamer from the 5 ' to 3 ' end was 28 mers long: GCACTCTGTGGGGGTGGACGGGCCGGGT; it was ordered from Eurofins and 
stored at $-20^{\circ} \mathrm{C}$. The aptamer was unconjugated with no modifications and already in a reconstituted solution of $43.79 \mathrm{ug} / \mathrm{uL}$. Three concentrations of the anti-VEGF-A DNA aptamer were made: 100nM, 50nM, and 25nM. The 100nM solution was made by pipetting $1 \mathrm{uL}$ of the $43.79 \mathrm{ug} / \mathrm{uL}$ solution into $48.996 \mathrm{~mL}$ of DI $\mathrm{H}_{2} \mathrm{O}$. . The $50 \mathrm{nM}$ concentration was made by pipetting $1 \mathrm{~mL}$ of the $100 \mathrm{nM}$ solution into $1.5 \mathrm{~mL}$ of $\mathrm{DI} \mathrm{H}_{2} \mathrm{O}$. The $25 \mathrm{nM}$ solution was made by pipetting $1 \mathrm{~mL}$ of the $100 \mathrm{nM}$ solution into $3 \mathrm{~mL}$ of $\mathrm{DI} \mathrm{H}_{2} \mathrm{O}$. The solutions were stored at $2-4^{\circ} \mathrm{C}$.

\section{pH of VEGF-A Solution}

The $\mathrm{pH}$ of the VEGF turned out to be an important factor for the immobilization of the VEGF onto the CM-Dextran. Initially after the 50nM VEGFA solution was mixed the $\mathrm{pH}$ was $\sim 7.0$. However due to the isoelectric point of both the VGEF-A and the CM-Dextran, at this pH the VEGF-A was not able to immobilize on the CM-Dextran surface. Ueda et al. performed an experiment with VEGF-A and used a pH of 4.5 [39], so the $\mathrm{pH}$ of the solution was adjusted accordingly. A $20 \mathrm{mM}$ solution of $\mathrm{HCl}$ was made to adjust the $\mathrm{pH}$ of the VEGF-A solution as well as a pH probe. To make the $20 \mathrm{mM} \mathrm{HCl}$ solution $10 \mathrm{uL}$ of $6 \mathrm{M} \mathrm{HCl}$ was added to $2990 \mathrm{uL}$ of $\mathrm{DI} \mathrm{H}_{2} \mathrm{O}$. Initially this process was carefully calculated out but the 1M PBS buffer resisted the change in $\mathrm{pH}$ so much more $\mathrm{HCl}$ had to be added to reach the desired $\mathrm{pH}$. The $\mathrm{pH}$ of both the $25 \mathrm{nM}$ and 150nM VEGF-A solutions were adjusted to $\sim 4.5$. 


\section{Other solutions}

To regenerate the surface of the VEGF-A functionalized CM-Dextran chip a $20 \mathrm{mM}$ solution of $\mathrm{NaOH}$ was used. A $1 \mathrm{M} \mathrm{NaOH}$ solution made by adding 1 gram $\mathrm{NaOH}$ to $1 \mathrm{~L} \mathrm{DI} \mathrm{H}_{2} \mathrm{O}$; this solution was then diluted down to $20 \mathrm{mM}$.

\section{EXPERIMENTS}

Before any experimental SPR runs were carried out all solutions utilized were degassed for 1-2 hours to remove any dissolved gases in the solutions. The machine was also cleaned and prepared with the same steps as in the $1 \%$ ethanol calibration experiment. CM-Dextran chips were used and 1M PBS running buffer. The flow rate of each experiment varied.

\section{$\underline{\text { Run } 1}$}

The first SPR run used the $\mathrm{pH} \sim 7.0$ 50nM VEGF-A solution, and the antiVEGF-A antibody solutions and the anti-VEGF-A DNA aptamer solutions. The surface of the CM-Dextran was prepared for immobilization by using an activation kit bought from Biosensing Instrument. For this run all activation solutions were mixed before degassing took place. A $0.1 \mathrm{M} \mathrm{N}$ hydroxysulfosuccinimide (NHS) solution was made by adding $0.5 \mathrm{~mL} \mathrm{DI} \mathrm{H}_{2} \mathrm{O}$ to the provided vial. A 0.4M 1-(3-dimethylaminopropyl)-3-ethylcarbodiimide (EDC) solution was made by adding $0.5 \mathrm{~mL} \mathrm{DI} \mathrm{H}_{2} \mathrm{O}$ to the provided vial. A $1.0 \mathrm{M}$ Ethanol Amine (EA) solution was also made by adding $1 \mathrm{~mL}$ of $\mathrm{DI} \mathrm{H}_{2} \mathrm{O}$ to the provided vial. After all solutions were degassed and the SPR machine was loaded with running buffer and a new CM-Dextran chip and completely warmed up the CMDextran chip was activated and the VEGF-A was immobilized. The flow rate of 
the running buffer was set to $30 \mathrm{uL} / \mathrm{min}$. The $0.1 \mathrm{M} \mathrm{NHS}$ and $0.4 \mathrm{M}$ EDC were mixed together and immediately $100 \mathrm{uL}$ were injected into the flow to activate the CM-Dextran surface. Immediately after the activation signal finished, the $50 \mathrm{nM}$ VEGF-A solution was injected followed by the 1M EA solution in order to block the CM-Dextran surface. This whole process was performed in serial mode. The order of the injections was: $50 \mathrm{nM}$ antibody, $\mathrm{NaOH}, 50 \mathrm{nM}$ aptamer, $\mathrm{NaOH}$, 100nM antibody, $\mathrm{NaOH}, 25 \mathrm{nM}$ antibody, $\mathrm{NaOH}, 50 \mathrm{nM}$ antibody,

\section{$\underline{\text { Run } 2}$}

To help the VEGF-A immobilize to the CM-Dextran surface the $\mathrm{pH}$ of the VEGF-A solution was changed to 9. The isoelectric point of VEGF-A was either 8.5 [40] or 9.22 [41] so a pH between the two was chosen. The surface of the CM-Dextran was prepared for immobilization by using the activation kit bought from Biosensing Instrument. For this run the activation solutions were mixed after degassing took place and immediately before injection into the machine. Once the NHS/EDC solution was used to activate the CM-Dextran solution, the $25 \mathrm{nM}$ VEGF-A solution at pH 9 was injected a few times. The order of the injections was: $50 \mathrm{nM}$ antibody, $\mathrm{NaOH}, 50 \mathrm{nM}$ aptamer, $\mathrm{NaOH}, 100 \mathrm{nM}$ antibody, $\mathrm{NaOH}$, $25 \mathrm{nM}$ antibody, $\mathrm{NaOH}, 50 \mathrm{nM}$ antibody, $\mathrm{NaOH}, 100 \mathrm{nM}$ antibody, $\mathrm{NaOH}, 100 \mathrm{nM}$ antibody. All injections after immobilization took

\section{$\underline{\text { Run } 3}$}

The third SPR run used the $\mathrm{pH} \sim 4.5$ 25nM VEGF-A solution, and the antiVEGF-A antibody solutions and the anti-VEGF-A DNA aptamer solutions. The surface of the CM-Dextran was prepared for immobilization by using the 
activation kit bought from Biosensing Instrument. For this run all activation solutions were mixed after degassing took place. The CM-Dextran surface was activated with NHS/EDC solution and then three injections of VEGF-A at $\mathrm{pH} 4.5$ and one injection of VEGF-A at 9.3 were run. The CM-Dextran chip was then blocked using the EA solution. The order of the injections was: 50nM antibody, $\mathrm{NaOH}, 50 \mathrm{nM}$ antibody, 50nM antibody, $\mathrm{NaOH}, 100 \mathrm{nM}$ antibody, $\mathrm{NaOH}, 100 \mathrm{nM}$ aptamer, $\mathrm{NaOH}, 50 \mathrm{nM}$ aptamer, $\mathrm{NaOH}, 25 \mathrm{nM}$ antibody, $\mathrm{NaOH}$. After the final $\mathrm{NaOH}$ a few other injections were carried out but they were distorted by an air bubble in the system and therefore discounted. All injections after immobilization took place were performed in single mode.

\section{$\underline{\text { Run } 4}$}

Since the prior SPR run did not allow for the kinetic analysis software to be used another SPR run was performed. In order to quantify the data the two channels had to be calibrated to give the same singles, this was achieved through using a 0.5M PBS buffer solution and Equation 2. The average in the initial channel readings was taken and used as the expected value. Once the calibration was finished the CM-Dextran surface was activated with NHS/EDC solution and three injections of $\mathrm{pH} 4.525 \mathrm{nM}$ VEGF-A was injected. After immobilization of VEGF-A, EA was injected to block the CM-Dextran. Only channel 2 was immobilized with VEGF-A using single mode injections. $\mathrm{NaOH}$ was injected to remove any remaining VEGF-A molecules and then the machine was switched to serial mode and the sample injections were made. The order of 
the injections was: $100 \mathrm{nM}$ antibody, $\mathrm{NaOH}, 100 \mathrm{nM}$ antibody, $\mathrm{NaOH}, 100 \mathrm{nM}$ aptamer, $\mathrm{NaOH}$, and 100nM aptamer.

$\underline{\text { Run } 5}$

Since the antibody did not work in run 4 , the SPR run was a fifth time. A 0.5M PBS buffer calibration was done for the new dextran chip before immobilization took place. The surface of the CM-Dextran chip was activated with NHS/EDC solution and the $\mathrm{pH} \sim 4.5150 \mathrm{nM}$ VEGF-A solution was injected four times in single mode into channel 2. The EA solution was then injected to block the CM-Dextran and $\mathrm{NaOH}$ was used to wash away any remaining VEGF-A molecules. The order of the injections was: $100 \mathrm{nM}$ aptamer, $\mathrm{NaOH}, \mathrm{NaOH}$, 100nM antibody, $\mathrm{NaOH}, 80 \mathrm{nM}$ antibody, and $\mathrm{NaOH}$. At this point an air bubble disrupted the experiment to the point it could not be continued.

\section{ANALYSIS SOFTWARE}

The BI-Data Program allows for analysis of the results recorded by the computer during the SPR runs. Selected data is transferred from the BI-Control to the data analysis program where several steps were performed with it. The program can zero the data responses, crop data, align curves, subtract reference responses, and calculate the kinetics of the data. First the curves to be analyzed were selected, one as reference (the channel where no VEGF-A was immobilized) and another (the channel where the VEGF-A was immobilized). The data was then zeroed to align the curves and cropped to the area of interest. Next the reference channel was subtracted from the other channel to give the actual interaction curve between the VEGF-A and the anti-VEGF-A antibody and 
anti-VEGF-A aptamer. Next the kinetic analysis was used to fit a curve to the data from which $k_{m}, k_{a}$, and $k_{d}$ were calculated, as well as $R_{\max }$. For the ethanol dilutions an additional step was added that allowed for an affinity curve to be calculated using the various concentrations. 


\section{RESULTS}

\section{Ethanol Dilutions}

The ethanol dilutions were used to both calibrate the SPR machine and to get a feel for the experimental process and analysis software.

Figure 5 shows the relationship between each of the ethanol

dilutions. The mDeg difference for the $1 \%$ ethanol solution was used in the calibration of channels by being fit to a $60 \mathrm{mDeg}$ curve. The $0.25 \%$ ethanol solution

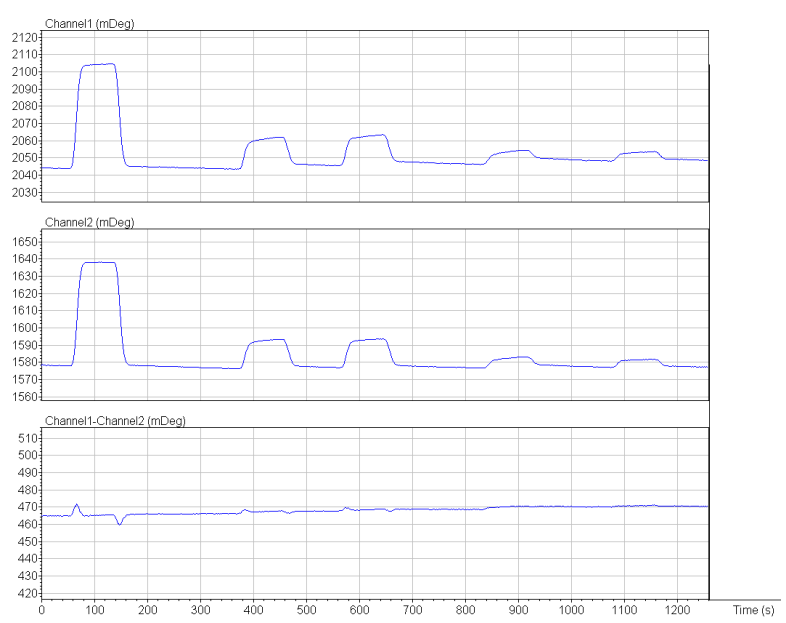

Figure 5: Ethanol dilutions Shows the ethanol dilutions from left to right: $1 \%$, $0.25 \%, 0.25 \%, 0.0625 \%$ and, $0.0625 \%$.

when measured in both channel one and channel two was equal to $\sim 15.41 \mathrm{mDeg}$. The $0.0625 \%$ ethanol solution had a change of $4.2 \mathrm{mDeg}$ when averaged across the two channels.

These three concentration curves were put into the software analysis program and the kinetics and affinity relationships between the ethanol and the gold surface of the chip were calculated two ways. The program was first used to find the kinetics: a line was fit to the binding curve from which the binding affinity $\left(K_{D}\right)$, dissociation rate constant $\left(k_{d}\right)$, association rate constant $\left(k_{a}\right)$, maximum response $\left(R_{\max }\right)$, and mass transport coefficient $\left(k_{m}\right)$ are calculated (Figure 6). $K_{D}=29.701 m M . R_{\max }=2,395 \cdot 5 . k_{a}=53.15 . k_{d}=1.579 . k_{m}=8881.8$. The second method used the affinity analysis to find the $K_{D}$ based on the steady state of each 


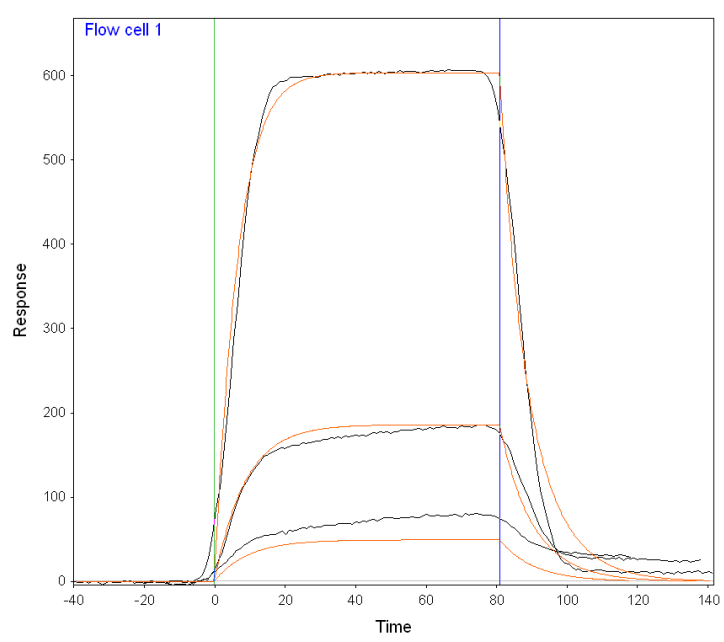

Figure 6: SPR analysis fit curves Shows the fit of the curve to the ethanol concentrations, those for $1 \%$ and $0.25 \%$ are much better than for $0.0625 \%$. From these curves the kinetic binding between the gold and the ethanol can be determined.

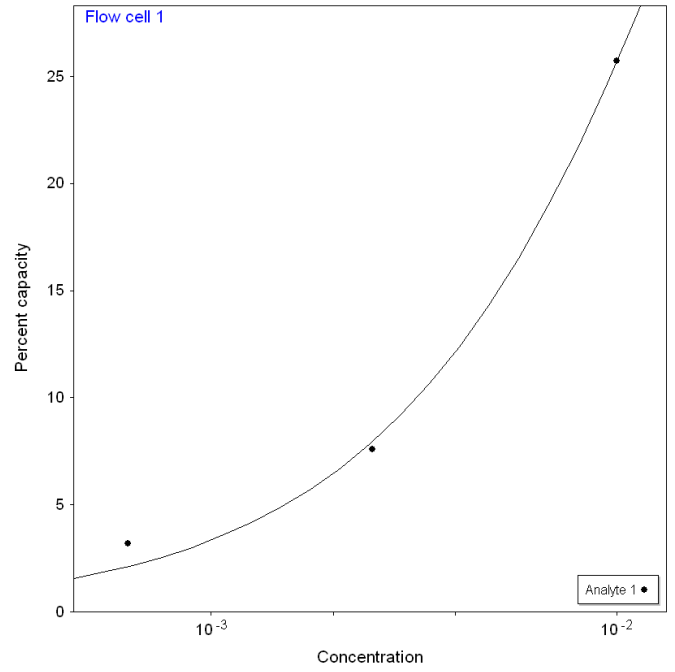

Figure 7: Affinity of ligand curve Shows the affinity curve between the ethanol and the gold chip surface, calculated using the concentrations of the ethanol.

curve between the different calibrations (Figure 7). From this method $K_{D}=$

29.2mM. $R_{\max }=2300.1$.

\section{Run 1}

The first SPR run for analysis of the kinetics between the VEGF-A and the anti-VEGF-A antibody and anti-VEGF-A aptamer (Figure 8). There was no binding between the molecules observed. The large downward curve was caused by the bulk flow of the liquid in the injected sample, the portion were the binding would have occurred is the bottom of the curve. If interaction had

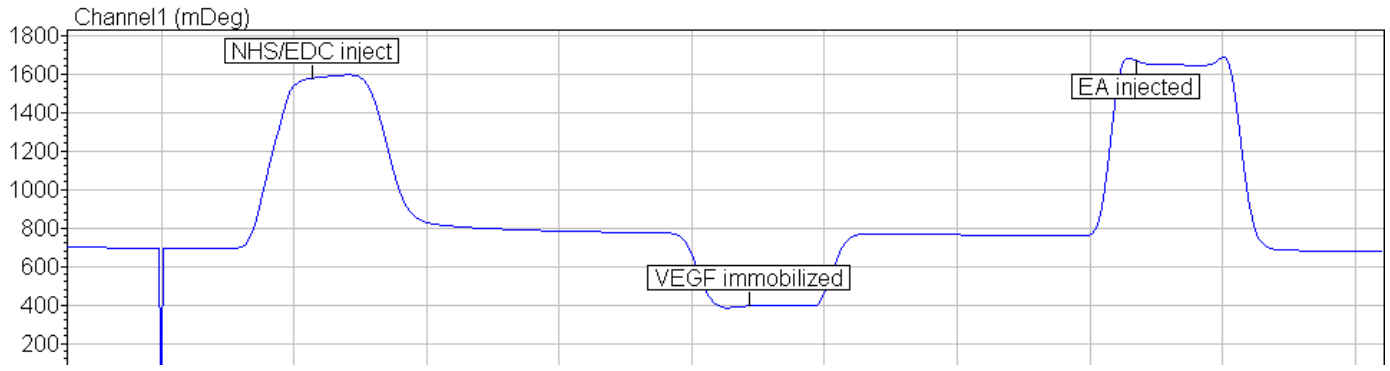

Figure 8: Attempted immobilization of VEGF-A Shows the activation of the CM-Dextran surface with NHS/EDC solution, the injection of the 50nM VEGF-A solution, and the deactivation of the surface with EA. 


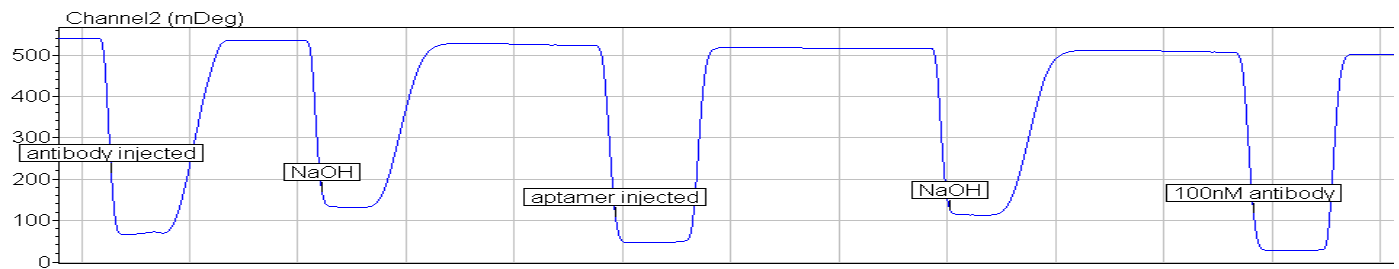

Figure 9: Absence of VEGF-A binding between antibody and aptamer

Shows an excerpt of the inaction curves between the VEGF-A and the anti-VEGF aptamer and the anti-VEGF-A antibody. Since the bottom of the curve is flat, no binding took place.

occurred, there would have been a slight increase in the line, however it was flat

for all sample injections, the $\mathrm{NaOH}$, and even $\mathrm{DI} \mathrm{H}_{2} \mathrm{O}$ (Figure 9).

\section{Run 2}

The second SPR run was changed from the first by adjusting the $\mathrm{pH}$ of the VEGF-A solution from $~ 7.0$ to $~ 9.0$. Even with this adjustment the VEGF-A did not immobilize onto the CM-Dextran surface (Figure 10). The VEGF-A upon injection had no interaction with the surface.

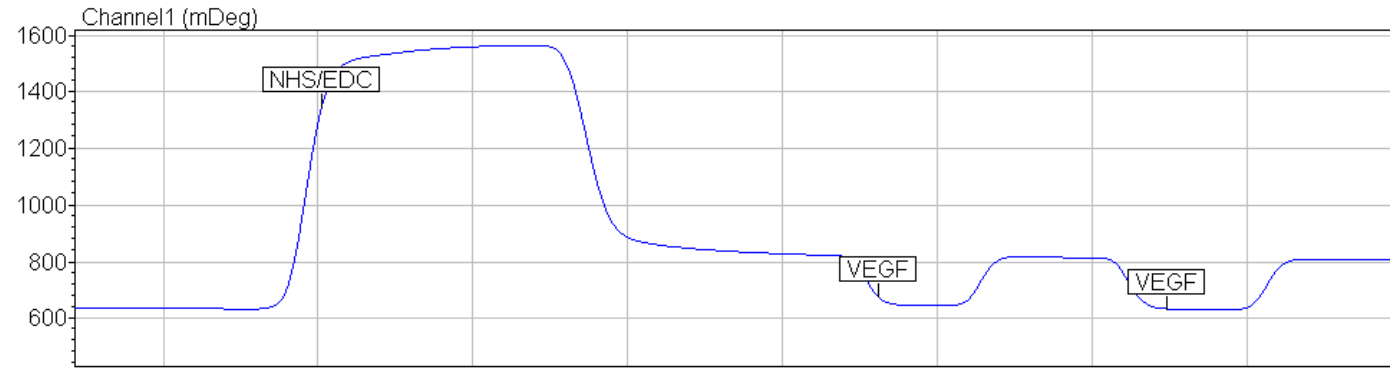

Figure 10: Failed immobilization of VEGF-A

Shows the activation of the CM-Dextran surface, however, the VEGF-A was not immobilize onto the surface, evidenced by the lack of an upward signal when the VEGF-A was injected.

\section{Run 3}

The third SPR run adjusted the $\mathrm{pH}$ of the VEGF-A again, this time to 4.5 . The VEGF-A was successfully immobilized onto the CM-Dextran as seen by the slight upward curve in the SPR signal after the initial bulk flow curve of the fluid (Figure 11). Due to the fact that channel 1 and channel 2 were not correctly calibrated, the kinetics on the molecular binding could not be analyzed. However 
a qualitative analysis of the

binding curves may be

done. Using the computer

the mDeg change in each

curve was measured

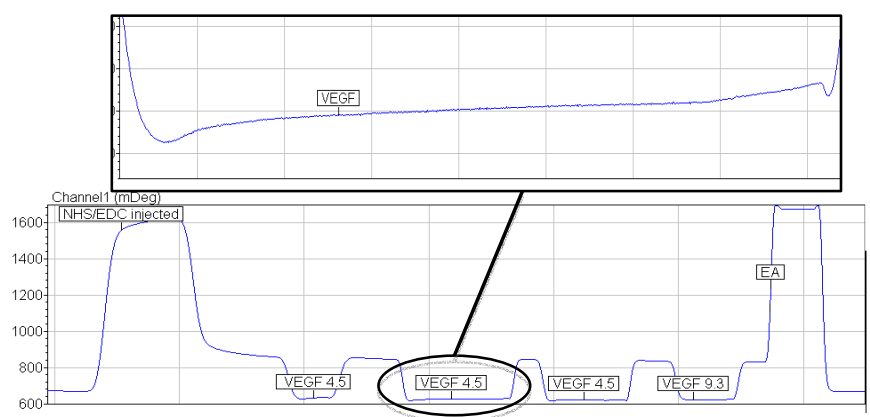

Figure 11: Successful immobilization of VEGF-A

(Figure 12).

Shows the immobilization of the VEGF-A onto the CMDextran. The large downward curve is caused by the bulk flow of the fluid in the sample since it is not the same as the running buffer. The immobilization of the VEGF-A is seen by the slight upward curve magnified

a

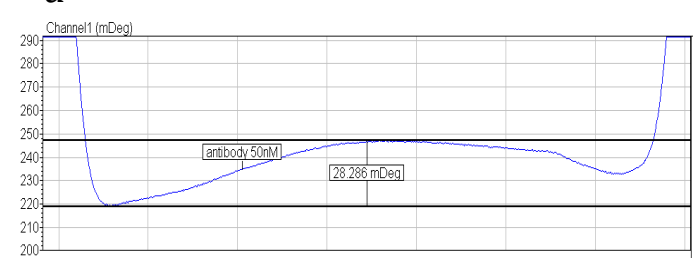

$\mathrm{b}$

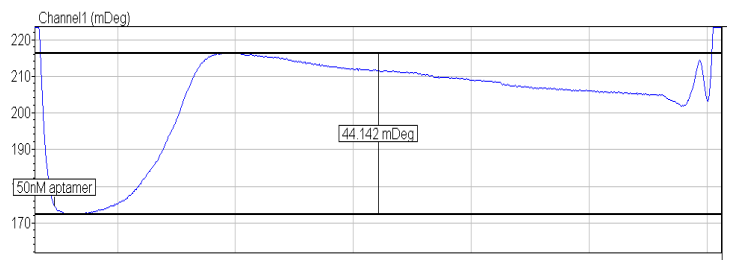

Figure 12: Quantitative kinetic analysis of antibody and aptamer a. Shows the binding curve between the anti-VEGF-A antibody and the VEGF-A. b. Shows the binding curve between the anti-VEGF-A aptamer and the VEGF-A. These curves simply show there was binding between the molecules.

\section{Run 4}

The fourth SPR run was performed to obtain data that would allow for quantitative kinetic analysis (Figure 13). A gold chip was used to obtain a new calibration where channel $1=2034.93$ and channel $2=2090.77$ (Table 1 ). Using the new calibrations 0.5M PBS buffer was used to calibrate the $\mathrm{CM}$ -

Dextran chip using the same mathematical process. The initial mDeg reading in channel 1 was

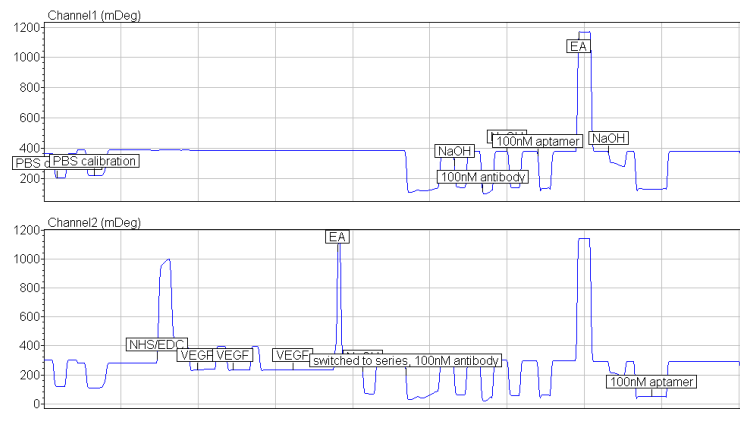

Figure 13: PBS buffer calibration Shows the overall SPR run from calibration to injections of the antibody and aptamer.

158.160 and in channel 2 was

184.557. The average was taken to find the 'ideal' number from which both 


\begin{tabular}{|c|c|c|c|}
\hline Channel圈圆 & $\begin{array}{c}3000(60 / 89.143)=\text { 罒 } \\
2019.23 \text { ? }\end{array}$ & $\begin{array}{c}2019.23(60 / 59.537)=? \\
2034.93 \text { ? }\end{array}$ & ? \\
\hline Channel[2] & $\begin{array}{c}3000(60 / 85.815)=\text { 爾 } \\
2097.54 \text { ? }\end{array}$ & $\begin{array}{c}2097.54 \text { ( } 60 / 61.667)=\text { ? } \\
2040.83 \text { ? }\end{array}$ & $\begin{array}{c}2040.83(60 / 56.280)= \\
2175.73 \text { ? }\end{array}$ \\
\hline
\end{tabular}

Table 1: PBS calibration calculations

Shows the calibrations calculations for $1 \%$ ethanol to reach $60 \mathrm{mDeg}$ change in both channel 1 and channel 2 . Once calibrated the channels can be used for quantitative data where one channel is a reference to subtract out bulk flow interference.

channels were to be calibrated: average $=171.359$. The same calibrate mathematical process was carried out as with the $1 \%$ ethanol and channel $1=$ 2171.73 and channel $2=1966.89$. The surface appeared to be functionalized, giving a curve similar to Graph 7. Once the injections of anti-VEGF-A antibody and anti-VEGF-A aptamer were completed the kinetic analysis program was used to analyze the data. Channel 2 was where the VEGF-A was immobilized and channel 1 was used for the reference, the data was imputed into the program (Figure 14). The curve was zeroed to align the baselines of both channel 1 and channel 2 (Figure 15). Excess data was removed using the cropping page in the software. The date was then aligned. The reference,

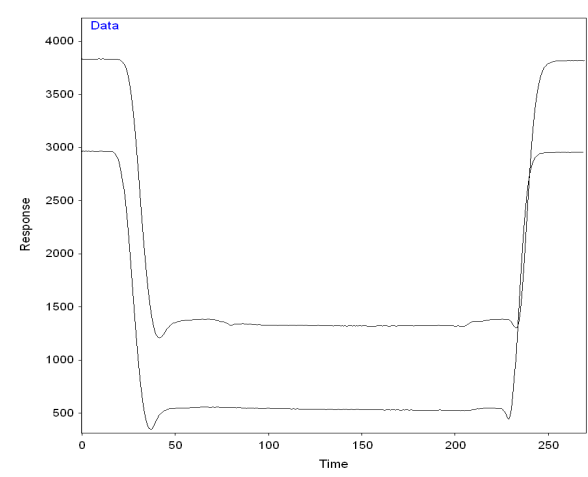

Figure 14: Step 1 of kinetic analysis Data is input into program in order to determine the binding interactions between the VEGF-A and the antiVEGF-A ssDNA aptamer. a.
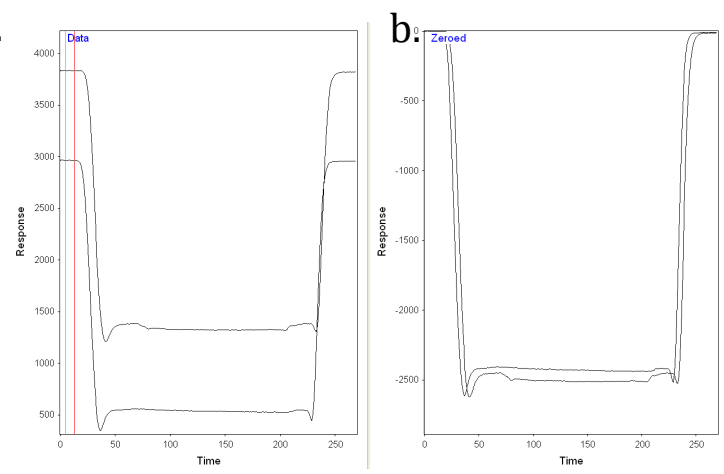

Figure 15: Step 2 of the kinetic analysis The program zeros the curves so that they can be directly compared. a. Shows the graph before zeroing took place. b. Shows the graph after it is zeroed. 
a.

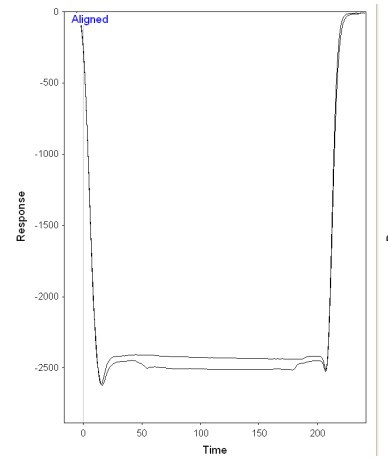

b.

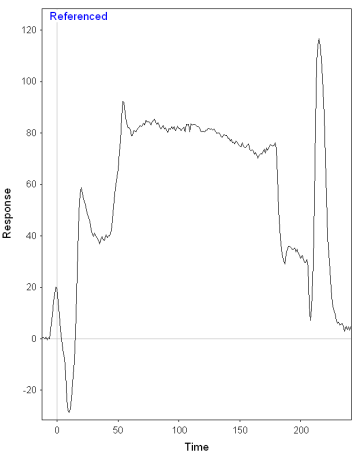

Figure 16: Step 5 of kinetic analysis Program subtracted the reference channel from the channel where the VEGF-A was immobilized. a. Shows the graph before it is referenced. b. Shows the graph after it is referenced, can finally see the binding curve between the VEGF-A and the anti-VEGF-A DNA aptamer.

channel 1 , was subtracted from channel 2 to reveal the actual binding interaction curve between VEGF-A and anti-VEGF-A aptamer (Figure 16). A curve was fit to the curve from which the kinetic data was found (Figure 17). From the data obtained, analyzed, and averaged: $\mathrm{K}_{\mathrm{D}}=24.1 \mathrm{nM}, \mathrm{k}_{\mathrm{m}}=$

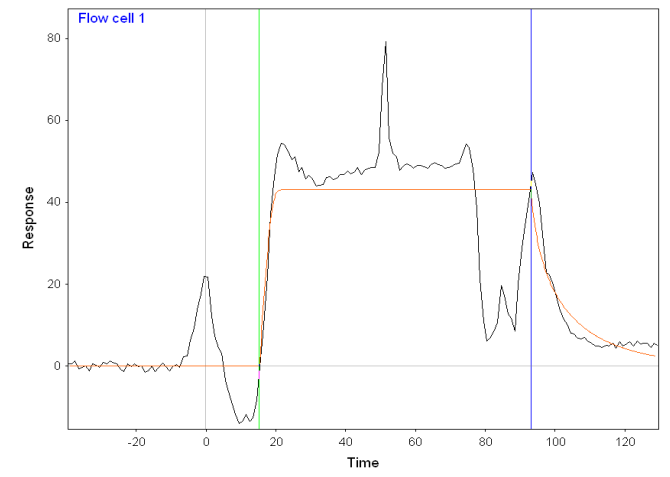

Figure 17: Kinetic curve of aptamer Step 6 of the kinetic analysis program fits a line to the binding curve. From this curve the $k_{m}, k_{a}, k_{d}, R_{\max }$, and $K_{D}$ are calculated.

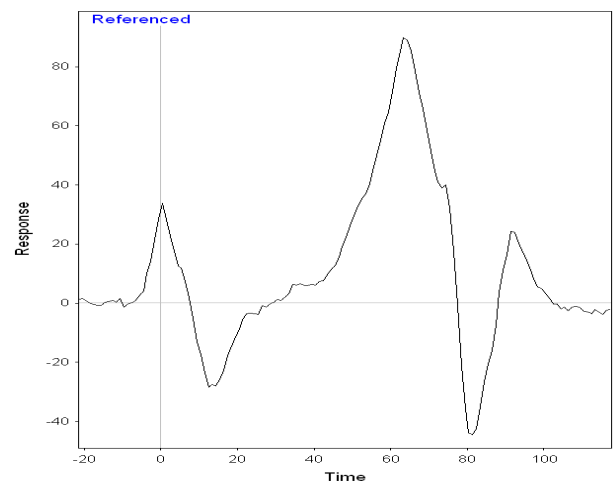

Figure 18: Curve of denatured antibody Shows the fitted line to the binding curve of the interaction between the antiVEGF-A and VEGF-A.

$1.76 \times 10^{8}, k_{a}=1.02 \times 10^{11}, k_{d}=2549$, and $R_{\max }=56.7$ (Table 2). The 100nM

antibody was also analyzed using the kinetic software (Figure 18).

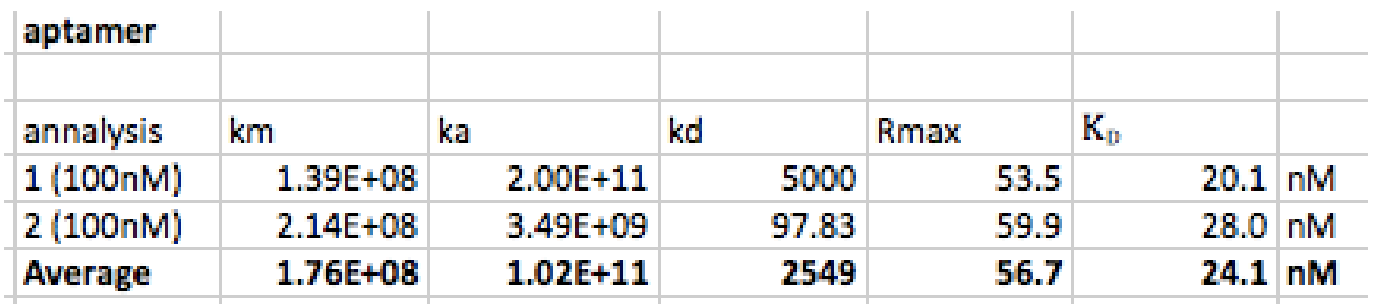

Table 2: Aptamer Kinetics

Shows the results of the kinetic analysis software for the anti-VEGF-A aptamer. 


\section{Run 5}

Run 5 was done to analyze the kinetics of the anti-VEGF-A antibody, all of the same steps were performed as with the aptamer until the software fitted a line to the binding curve (Figure 19). This graph was used to find the kinetic properties between the VEGF-A and the anti-VEGF-A antibody: $\mathrm{K}_{\mathrm{D}}=88.4 \mathrm{uM}, \mathrm{k}_{\mathrm{m}}=$ $2.16 \times 10^{8}, k_{a}=1.75 \times 10^{5}, k_{d}=14.78$, and $\mathrm{R}_{\max }=74,617$ (Table 3).

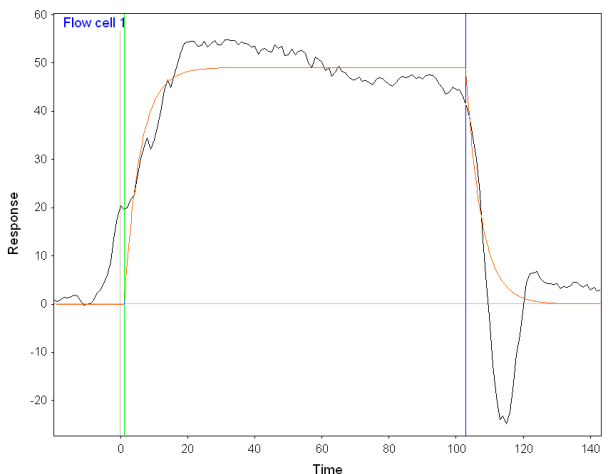

Figure 19: Kinetic curve of antibody Shows the fitted line to the binding curve of the interaction between the anti-VEGF-A and VEGF-A.

\begin{tabular}{|l|r|r|r|r|r|r|r|}
\hline antibody & & & & & & & \\
\hline & & & & & & \\
\hline analysis & $\mathrm{km}$ & $\mathrm{ka}$ & $\mathrm{kd}$ & $\mathrm{Rmax}$ & $\mathrm{K}_{0}$ & & \\
\hline $1(100 \mathrm{nM})$ & $1.89 \mathrm{E}+08$ & $2.53 \mathrm{E}+05$ & 30.81 & 88726 & 122 & $\mathrm{uM}$ \\
\hline $2(100 \mathrm{nM})$ & $3.17 \mathrm{E}+08$ & $8.15 \mathrm{E}+04$ & 10.31 & 124800 & 127 & uM \\
\hline $3(80 \mathrm{nM})$ & $1.41 \mathrm{E}+08$ & $1.92 \mathrm{E}+05$ & 3.219 & 10326 & 16.8 & uM \\
\hline Average & $\mathbf{2 . 1 6 E + 0 8}$ & $1.75 \mathrm{E}+05$ & 14.78 & $\mathbf{7 4 6 1 7}$ & $\mathbf{8 8 . 4}$ & uM \\
\hline
\end{tabular}

Table 3: Antibody kinetics

Shows the results of the kinetic analysis of the anti-VEGF-A antibody. 


\section{DISCUSSION}

\section{ETHANOL}

The $1 \%$ ethanol gave a measurement of $60 \mathrm{mDeg}$ after several calibrations of both channel 1 and channel 2 . The $0.25 \%$ ethanol was $1 / 4$ the concentration of the $1 \%$ ethanol so it was expected to give a response of $15 \mathrm{mDeg}$. The actual response was $\sim 15.41 \mathrm{mDeg}$ which was very close to the expected. The $0.0625 \%$ ethanol solution was $1 / 4$ of the $0.25 \%$ and $1 / 16$ of the $1 \%$ solution, so the expected $\mathrm{mDeg}$ response was 3.75 . The actual results were $\sim 4.2 \mathrm{mDeg}$. This difference was later explained by the fact that the micropipette used in the mixture of the $0.0625 \%$ solution was not correctly calibrated, causing the solution to be more concentrated that it should have been. It is possible to tell that the solution was more and not less concentrated than it should have been because the mDeg change was greater than it should have been and not less.

The calibration and dilution experiment was important because the instructions and results were clearly given the $\mathrm{BI}-2000$ Manual and therefore it was easy to tell if the experiment had been performed correctly through use of the SPR machine. This was an essential step to know before moving onto the more complicated experiments where the materials used were much more expensive. Mistakes such as the calibration of the micropipette were found prior to the following experiments and new one was ordered. Several other SPR runs were done using gold chips and ethanol for further experience with the equipment and techniques such as the correct amount of immersion oil to be used were learned. 
The ethanol dilution experiment also allowed for the kinetic analysis software to be used, when unfamiliar with the steps of the program it is confusing and the results do not make sense. Using the ethanol dilutions allowed for analysis using both the affinity and the kinetic portions of the software. From the affinity analysis it was found that $K_{D}=29.2 \mathrm{mM}$ and $R_{\max }=2300.1$. When using the kinetic analysis it was found that $K_{D}=29.701 \mathrm{mM}, R_{\max }=2,395.5, k_{a}=53.15$, $k_{d}=1.579$, and $k_{m}=8881.8$. While the kinetic analysis results in more data, it is possible to compare the $K_{D}$ and $R_{\max }$ directly between the two methods both of which were very close between the two. The differences between these two techniques was that the affinity analysis required the use of multiple dilutions of a sample and then the software was used to compare the steady-state binding at each concentration. The kinetic analysis requires only one sample with a known concentration to be used and provides more information. Ideally both methods would be used for every experiment since the results for each can be used to verify each other.

The $K_{D} \approx 29 \mathrm{mM}$ means that there is weak binding between the gold surface and the ethanol, most likely due only hydrogen bonding between the two as the ethanol is adsorbed to the gold surface as the fluid travels over it. Ideally for the binding between a target molecule and its binder, the $K_{D}$ should be as small of a number as possible. For something such as the interaction between streptavidin and biotin the $K_{D}$ is on the order of $f M$ [42], which is equal to $10^{-15} M$. 


\section{VEGF-A AND ANTI-VEGF-A POLYCLONAL ANTIBODIES SSDNA APTAMER}

\section{Run 1}

The first SPR run using CM-Dextran and VEGF-A resulted in no immobilization of the VEGF-A and therefore not interaction between it and the anti-VEGF-A aptamer or anti-VEGF-A polyclonal antibody solutions. This was determined by the fact that there was not positive interaction curve when the antibody or aptamer were injected into the 1M PBS buffer flow. The aptamer and antibody curves were identical, both showing the same downward bulk flow and not interaction between the VEGF-A molecules. The Biosensing Instruments technical expert was contacted and the negative results were verified it was suggested that the VEGF-A was not able to immobilize in the CM-Dextran due to the $\mathrm{pH}$ of the solution [43]. The technical specialist at Biosensing Instruments recommended that the experiment be performed within a $\mathrm{pH} \pm 1$ from the isoelectric point of the protein. The isoelectric point of VEGF-A was found to be either 8.5 or 9.2 so the $\mathrm{pH}$ of 9 was decided to be used since it was between the two pHs.

Anther change that was suggested by the technical specialist was to use the same solution as the buffer for that which the VEGF-A was in to decrease the downward bulk flow curve. Since the entire VEGF-A sample was already diluted to $50 \mathrm{nM}$ in $\mathrm{DI} \mathrm{H}_{2} \mathrm{O}$, the sample was further diluted to $25 \mathrm{nM}$. In completely optimized SPR runs using immobilized proteins concentrations as low at 10nM have been used for immobilization so the decrease in concentration has precedent. However, since the VEGF-A experiment could not be optimized as it 
would use too many materials and therefore cost too much, a higher concentration was suggested. In order to compensate for the use of only a $25 \mathrm{nM}$ solution of VEGF-A, multiple injections of samples were to be used to increase the possible amount of protein that could be immobilized.

It was determined that both the CM-Dextran chips and the chemicals in the activation kit were still viable, an important determination since the CMDextran chips were labeled 'for best use' before February and the experiment was being run in early April.

\section{Run 2}

Changing the $\mathrm{pH}$ of the VEGF-A solution from $\sim 7.0$ to 9.0 did not result in the immobilization of the VEGF-A into the CM-Dextran on the chip surface. When immobilizing a protein on the surface of CM-Dextran it is important to take into consideration not only the isoelectric point of the protein but of the CM-Dextran. When the $\mathrm{pH}$ of a solution containing a protein is less than the isoelectric of the protein the net charge of the protein is positive and when the $\mathrm{pH}$ is greater than the isoelectric point of the protein the net charge will be negative. This is important because the CM-Dextran surface is negative when the $\mathrm{pH}$ of the solution is greater than 3.5 , in order for the VEGF-A protein to be able to immobilize on the surface the $\mathrm{pH}$ should be higher than 3.5 but lower that the isoelectric point of the VEGF-A. In run 2, where the $\mathrm{pH}$ was 9.0, if the protein had the isoelectric point of 8.5 , that meant that it had a negative net charge and the CM-Dextran had a negative net charge. This would result in the repulsion of the VEGF-A from the surface of the CM-Dextran, inhibiting immobilization (Figure 
20). Using this

information, a new

$\mathrm{pH}$ of $\sim 4.5$ was

selected for the

VEGF-A solution to

complement

immobilization.
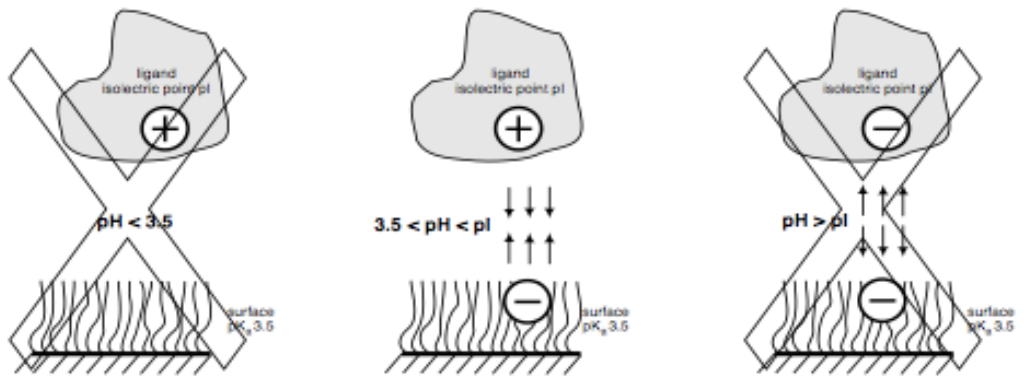

Figure 20: Immobilization of CM-Dextran

Shows the attraction or repulsion between a protein and the CMDextran surface depending on the $\mathrm{pH}$ of the solution. (Provided courtesy of GE Healthcare)

\section{Run 3}

In run 3, immobilization of the VEGF-A occurred, indicating that the $\mathrm{pH}$ of the VEGF-A solution was the problem inhibiting immobilization in run 1 and run 2. This immobilization was verified by the upward binding curve after the initial downward bulk flow of the VEGF-A solution was injected into the 1M PBS buffer flow. Due to the $25 \mathrm{nM}$ concentration three injections of the VEGF-A were used. In order to prove binding, an injection of pH 9.3 VEGF-A was also used, that did not immobilize. Due to the fact that the injections were only done in one channel, only a qualitative analysis could be done between the aptamer and the antibody. Using this initial analysis between the 50nM antibody and 50nM aptamer solutions: the $\mathrm{k}_{\text {on }}$ for the appeared to higher than that of the antibody. The aptamer also appears to have a slightly better affinity for the VEGF-A than the antibody does because there was a larger mDeg change, indicating a higher $\mathrm{K}_{\mathrm{D}}$. From the graphs it was not possible to analyze the $\mathrm{k}_{\text {off }}$ differences between the antibody and the aptamer. To obtain qualitative differences between the antiVEGF-A antibody and the anti-VEGF-A aptamer the SPR experiment had to be 
run again using a fully calibrated machine and using one channel as a reference to subtract the bulk flow from the actual interaction curve.

\section{Run 4}

The technical specialist at Biosensing Instruments was consulted on how to calibrate the SPR machine for CM-Dextran chips verse gold chips: using the initial calibration numbers obtained using a normal gold chip, a 50\% dilution of the running buffer should be used to further calibrate the CM-Dextran chip. Since a $1 \mathrm{M}$ PBS buffer was being used as the running buffer, a $0.5 \mathrm{M}$ PBS solution was used to calibrate for the specific CM-Dextran chip. This calibration was necessary because in order to use the kinetic analysis software, channel 1 and channel 2 need to give the exact same readings. This way, any differences between the two can be detected. This is achieved by immobilizing only one channel with the VEGF-A, that way only one channel will bind the anti-VEGF-A antibody or aptamer and the other channel will only show the interaction of the anti-VEGF-A antibody or aptamer with the surface of the CM-Dextran and any other bulk flow characteristics. When using a reference channel it is difficult to see any difference in the curve between the channel with the immobilized VEGF$A$ and that without. It is not until the data is manipulated using the kinetic analysis software that the binding curve becomes apparent. Each of the 100nM antiVEGF-A aptamer curves were put into the program so that an average of the interactions could be taken to get more accurate results. 
After the data was zeroed, aligned, cropped, and the reference channel subtracted a line was fit from which the software was able to calculate $K_{D}=$ 24.1 $\mathrm{nM}, \mathrm{k}_{\mathrm{m}}=1.76 \times 10^{8}, \mathrm{k}_{\mathrm{a}}=1.02 \times 10^{11}, \mathrm{k}_{\mathrm{d}}=2549$, and $\mathrm{R}_{\max }=56.7$. The $\mathrm{K}_{\mathrm{D}}$ from the literature where the anti-VEGF-A aptamer was taken was equal to $20 \mathrm{nM}$, so the $K_{D}$ found was very close. This difference could be explained by fact that the VEGF-A used in this experiment had a different source than that used in the literature. The VEGF-A used in this experiment came from HKE 293 derived cells. It was also composed of only amino acid 27-191 of the whole sequence that normally makes up VEGF-A. The VEGF-A used in the literature came from Sf21 insect cells and there was no data given for if it was composed of the whole polypeptide sequence. If any of the missing amino acids composed portions of the VEGF-A to where the aptamer bound, that would explain the change from $20 \mathrm{nM}$ to $24.1 \mathrm{nM}$. Either way since the equilibrium dissociation constant $\left(K_{D}\right)$ was in the nanomolar region, that means that the affinity between the VEGF-A and anti-VEGF-A aptamer was very good. Typically the equilibrium dissociation constant is between $1 \times 10^{-5}$ and $1 \times 10^{-12}$. The association rate constant, $k_{a}$, as suggested in the qualitative SPR run, at

$1.02 \times 10^{11} \mathrm{M}^{-1} \mathrm{~s}^{-1}$ is very

high. The association

rate constant is how well the VEGF-A and aptamer recognize each

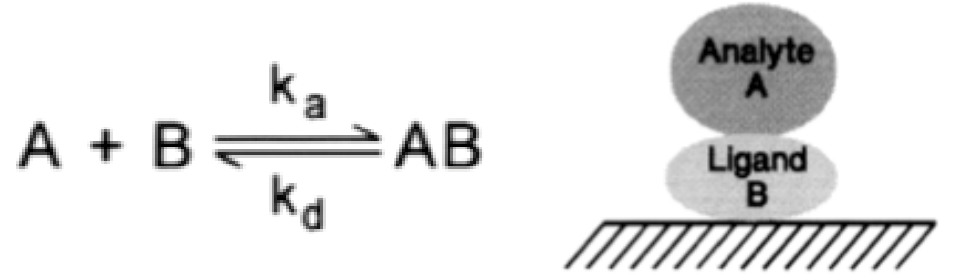

Figure 21: Kinetic equilibrium Shows the relationship between the VEGF-A (B) and the antiVEGF-A aptamer (A). The equation is for calculating the association rate constant and the dissociation rate constants. (Provided courtesy of GE Healthcare) 
other. Typical association rate constants are between $1 \times 10^{-3}$ and $1 \times 10^{7} \mathrm{M}^{-1} \mathrm{~s}^{-1}$. The dissociation rate constant at $2549 \mathrm{~s}^{-1}$ is much less than the association rate constant and it shows how stable the binding between the VEGF-A and the aptamer was. Typical dissociation rate constants are between $1 \times 10^{-1}$ and $5 \times 10^{-6}$ $\mathrm{s}^{-1}$. This suggests that the binding between the VEGF-A and the aptamer is not very stable, however the extremely high association constant overcomes this and still allows for an overall high equilibrium dissociation constant. $R_{\max }$ at 56.7 means maximum binding capacity and represents the total concentration of VEGF-A protein immobilized on the CM-Dextran chip.

When the anti-VEGF-A antibody was analyzed it was found that it had denatured and was no longer binding to the VEGF-A. This makes sense because the antibody solution was being stored at between $2^{\circ} \mathrm{C}$ and $8^{\circ} \mathrm{C}$ and on the manufacture's information sheet at that temperature the antibody was only viable for around two weeks. This experiment was performed roughly 3 weeks after the solution was made. Luckily, some of the $100 \mathrm{nM}$ antibody solution was being stored at $-20^{\circ} \mathrm{C}$, where it is stable for up to a year. However, this shows one of the limitations between using antibodies instead of aptamers, the aptamer was still stable even after three weeks of storage at a higher temperature. In some places where temperature control is not easily available this could be a huge incentive to use aptamers over antibodies. In order to determine exactly how much longer aptamers last than antibodies additional testing would be required for this particular antibody/aptamer grouping. 


\section{Run 5}

Since the antibody used in run 4 had denatured, another SPR run was performed to get usable data for direct comparison between the antibody and the aptamer. In order to ensure that the VEGF-A was still viable and that there was enough for the anti-VEGF-A antibody to bind to the $150 \mathrm{nM}$ solution was used. Using this concentration would provide ample binding sites to analyze the antibody; even at this concentration 4 injections of the VEGF-A were made. This precaution was also taken because the antibody had been giving another researcher problems with ability to bind with the VEGF-A. The results found were that $\mathrm{K}_{\mathrm{D}}=88.4 \mathrm{uM}, \mathrm{k}_{\mathrm{m}}=2.16 \times 10^{8}, \mathrm{k}_{\mathrm{a}}=1.75 \times 10^{5,} \mathrm{k}_{\mathrm{d}}=-14.78$, and $\mathrm{R}_{\max }=74,617$. The $R_{\max }$, is noticeably higher than that with the aptamer, this is due to the use of the 150 nM VEGF-A solution instead of the $25 \mathrm{nM}$ solution as over 1000 times the amount of VEGF-A was immobilized on the CM-Dextran surface. The $K_{D}$ at 88.4uM has a magnitude of 1000 times less affinity for VEGF-A than the antiVEGF-A aptamer. The association rate constant at $1.75 \times 10^{5} \mathrm{M}^{-1} \mathrm{~s}^{-1}$ for the antiVEGF-A antibody was also significantly less than that of the aptamer, meaning that the antibody did not recognize the VEGF-A as well. The $\mathrm{k}_{\mathrm{d}}$ for the antibody was $14.17 \mathrm{~s}^{-1}$ meaning that the stability between the VEGF-A and the anti-VEGFA antibody was higher than that between the VEGF-A and the aptamer, though still not low enough to truly be stable.

\section{Antibody verses aptamer}

Figure 22 shows the difference between high-affinity and lower-affinity curves. The anti-VEGF-A aptamer follow the high-affinity curve with a high $\mathrm{k}_{\mathrm{a}}$ and 
a high $\mathrm{k}_{\mathrm{d}}$. The anti-VEGF-A

antibody follows the lower-

affinity curve by having both a

low $\mathrm{k}_{\mathrm{a}}$ and a low $\mathrm{k}_{\mathrm{d}}$. Therefore

the aptamer had the overall

highest affinity for the VEGF-A

by a significant amount making

it the better option between the

two based on binding affinities.

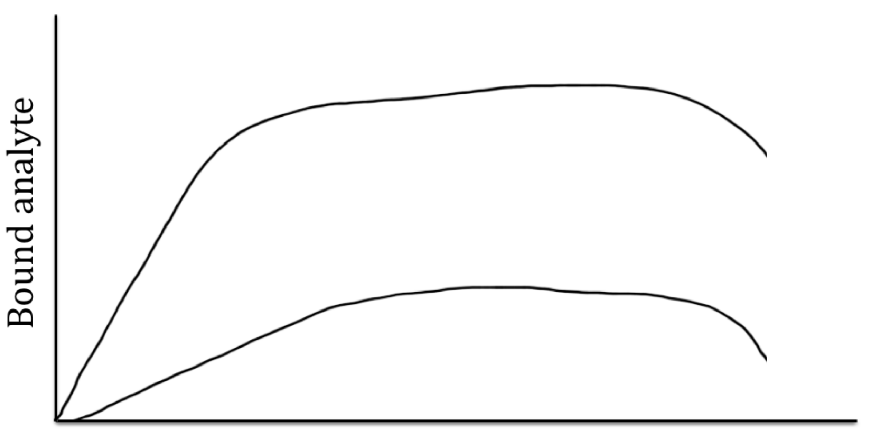

Time

Figure 22: High and low affinity curves The upper curve shows a high-affinity curve where $k_{a}$ is high and $k_{d}$ is high. The lower curve shows a lower-affinity curve where $k_{a}$ is low and $k_{d}$ is high. A high $\mathrm{k}_{\mathrm{a}}$ means there is fast association and a high $\mathrm{k}_{\mathrm{d}}$ means there is fast dissociation. (From Bio Rad "Protein Interaction Analysis")

While in this experiment the aptamer had a much higher affinity for the VEGF-A in some literature there have been antibodies modified to have affinities less than $1 \mathrm{nM}$ [33]. One possible reason that the affinity between the VEGF-A and the anti-VEGF-A antibody was so poor was because it was a polyclonal antibody solution; while the antibodies were created for VEGF-A there was no screening method to select for high affinity binding antibodies in the solution. As seen in Table 3, the $K_{D}$ is much lower in the $80 \mathrm{nM}$ solution that it was in the $100 \mathrm{nM}$ solution; perhaps this was because the $80 \mathrm{nM}$ solution had more antibodies with higher affinity for the VEGF-A than were in the 100nM solution. Ideally for direct comparison between aptamers and antibodies, a

\section{SPR for analysis}

SPR offers many advantages for protein and analyte binding analysis.

When compared to techniques such as ELISA and RIA (relative affinity index), SPR offs a much quicker methodology. In addition, there are no other laboratory 
techniques that offer the same level of kinetic analysis. Traditionally

spectrophotometers have been used to observe kinetics but not in the same realtime way. The fact that only $\mathrm{UL}$ of sample is required at concentrations in the $\mathrm{nM}$ range means that this method can be used for analyzing costly materials without high cost. 


\section{CONCLUSION}

Depending on what function the antibody or aptamer is to be used for will depend on which should be used, however, aptamers have the same function ability as antibodies do while offering easier modifications and more stability.

Antibodies have long been limited by the fact that they need to maintain conditions similar to those within the body. Temperature it one of the main methods through which antibodies are irreversibly denatured; this comes into paly when being used for diagnostic applications, for example, pregnancy tests have a shelf life of $12-24$ months when stored at room temperature (between $2^{\circ} \mathrm{C}$ and $30^{\circ} \mathrm{C}$ ). If a test is being created for in the field testing in a country with temperatures continually above $30^{\circ} \mathrm{C}$ where there is limited access to refrigeration then antibody assays are not going to function for their entire shelf life. Aptamers on the other hand can be regenerated from their denatured form and are stable in long-term storage and allow for transportation in various temperatures [45]. The temperature of denaturation for nucleic acid sequences depends on what order the nucleic acids is in, since aptamers that are engineered for a specific molecule can have different sequences, the one with the most stability in high temperatures can be selected in order to optimize it.

Another advantage of aptamers over antibodies is that they can be created to molecules that are toxic to cells. This allows for applications of the aptamer to be expanded beyond that of antibodies to testing devices for toxic chemicals within the environment. Antibodies during their production cannot be produced to something that will kill the cell or animal it is being produced in, since 
aptamers are produced in the lab without the use of cells they do not face this problem. Labeled aptamers could be used to determine the purity of a water sample by creating a group of aptamers that testing for certain bacteria, heavy metals or other contaminants and that will activate if bound.

Due to the high level of modifications for aptamers, they offer to be and ideal molecules for cell targeting. The process of Counter-SELEX has already been used to develop aptamers that will target only cells with cancer, while leaving alone those that are healthy. Since aptamers can be modified, there is potential for them to be used for targeted drug delivery of chemotherapy to cancerous cells, eliminating many of the negative side effects that accompany the traditional use of chemotherapy. A similar application of aptamers could result in the targeting of HIV infected cells and destroying them before spread to other T cells and the patient advances to AIDS. Another application would be to use differently labeled aptamers that bind to different types of cells for use in surgery, by creating differently fluoresced aptamers for different cell types it would be possible to avoid certain cell types, such as nerves to reduce damage to the body.

Future research that could apply the information learned about the kinetics of the anti-VEGF-A antibody and anti-VEGF-A aptamer. For example, lateral flow immunological assays could be developed using the aptamer, since its affinity is significantly better than the antibody. Monoclonal antibodies and MIPs should also be analyzed using SPR in order to directly compare with the results already obtained. Once all this information is ascertained, the molecules with the best 
binding could be used to create a portable, stable, and quick diagnostic assay. Similar testing could also be used to select for the best molecule choice in other diagnostics besides with VEGF-A. 


\section{BIBLIOGRAPHY}

1. Skoog, D. A.; Holler, F. J.; Crouch, S. R. Principles of Instrumental Analysis, 6th ed.; Thomson Higher Education, Belmont, CA, 2007. Biosensing Instrument. 102. SPR Sensitivity and Detection Limit.

2. Janeway CA Jr, Travers $P$, Walport M, et al. Immunobiology: The Immune System in Health and Disease. 5th edition. New York: Garland Science; 2001. The structure of a typical antibody molecule.

3. "The Nobel Prize in Physiology or Medicine 1984". Nobelprize.org. 12 May 2012 http://www.nobelprize.org/nobel_prizes/medicine/laureates/1984/

4. "Monoclonal Antibody Production" A Report of the Committee on Methods of Producing Monoclonal Antibodies. Institute for Laboratory Animal Research. National Research Council. National Academy Press. Washington, DC 1999.

5. Polyclonal and monoclonal: A comparison. Abcam. 1998-2012. http://www.abcam.com/index.html?pageconfig=resource\&rid=11269\&pid=11287 s $\mathrm{s}^{-1}$

6. Guidelines for Polyclonal Antibody Production in Laboratory Animals. Revised and approved May 2009. Berkeley. http://www.acuc.berkeley.edu/guidelines/antibody.pdf

7. Polyclonal Antibodies vs. Monoclonal Antibodies. Randox Life Sciences. http://www.randox-lifesciences.com/articles/57?articleSectionld=1

8. Mayo Clinic Staff. Monoclonal antibody drugs for cancer treatment: How they work. 1998-2012. Mayo Foundation for Medical Education and Research. http://www.mayoclinic.com/health/monoclonal-antibody/CA00082

9. Berg JM, Tymoczko JL, Stryer L. Biochemistry. 5th edition. New York: W H Freeman; 2002. Immunology Provides Important Techniques with Which to Investigate Proteins.

10. Borrebaeck, Carl A.K., "Antibodies in diagnostics- from immunoassays to protein chips." Review of Immunology Today, Vol. 21 No 8. (August) 379- 382.

11. Chen, Y., Christian Wiesmann, Germaine Fuh, Bing Li, Hans W. Christinger, Patrick McKay, Abraham M. de Vos, Henry B. Lowman. "Selection and Analysis of an Optimized Anti-VEGF Antibody: Crystal Structure of an Affinity-matured Fab in Complex with Antigen." J. Mol. Biol. (1999) 293, 865-881.

12. Jayasena, Sumedha. "Aptamers: An Emerging Class of Molecules That Rival Antibodies in Diagnostics." Clinical Chemistry (1999), 45:9, 1628-1650.

13. Batey, Robert T., Robert P. Rambo, Jennifer A. Doudna. "Tertiary Motifs in RNA Structure and Folding." Angew. Chem. Int. Ed. (1999), 38, 2326-2343.

14. Nowakoxski, Jacek, Ignacio Tinoco, Jr. "RNA Structure and Stability." Seminars in Virology (1997) 8, 153-165.

15. SantaLucia, Jr., John, Donald Hicks. "The Thermodynamics of DNA Structural Motifs." Annu. Rev. Biophys. Biomol. Struct. (2004), 33, 415-440.

16. Smirnov, Ivan, Richard H. Shafer. "Effect of Loop Sequence and Size on DNA Aptamer Stability." Biochemistry (2000), 39, 1462-1468.

17. Bishop, G. Reid, Jinsong Ren, Brandon C. Polander, Benjamin D. Jeanfreau, John O. Trent, Jonathan B. Chaires. "Energetic basis of molecular recognition in a DNA aptamer." Biophysical Chemistry 126 (2007) 165-175.

18. Wang, Ke Yu, Steven H. Krawczyk, Norbert Bischofberger, S. Swaminathan, Philip $\mathrm{H}$. Bolton. "The Tertiary Structure of a DNA Aptamer Which Binds to and Inhibits Thrombin Determines Activity." Biochemistry (1993), 32, 11285-11292. 
19. Ruigrok, Vincent J., Mark Levisson, Michel H. Eppink, Hauke Smidt, John Van Der Oost. "Alternative affinity tools: more attractive than antibodies?" Biochem. J. (2011). 436, 1-13.

20. Tombelli, Sara. Maria Minunni, Marco Mascini. Aptamers-based assays for diagnostics, environmental and food analysis. Bimolecular Engineering 24 (2007). 191-200.

21. Golden, M. C., Collins, B. D., Willis, M. C., Koch, T. H., 200. "Diagnostic potential of PhotoSELEX-evolved ssDNA aptamers. J. Biotechnol. 81, 167-178.

22. Etgar Levy-Nissenbaum, Aleksandar F. Radovic-Moreno, Andrew Z. Wang, Robert Langer, and Omid C. Farokhzad. Nanotechnology and aptamers: applications in drug delivery. Trends in Biotechnology Vol. 26 No.8 (2008). 442-449.

23. Christopher K. Dyke, MD; Steven R. Steinhubl, MD; Neal S. Kleiman, MD; Richard O. Cannon, MD; Laura G. Aberle, MS; Min Lin, PhD; Shelley K. Myles, BS, RN; Chiara Melloni, MD; Robert A. Harrington, MD; John H. Alexander, MD; Richard C. Becker, MD; Christopher P. Rusconi, PhD. First-in-Human Experience of an Antidote-Controlled Anticoagulant Using RNA Aptamer Technology : A Phase 1a Pharmacodynamic Evaluation of a Drug-Antidote Pair for the Controlled Regulation of Factor IXa Activity. Circulation 114 (2006). 2490-2497.

24. Yan Du, Chaogui Chen, Ming Zhou, Shaojun Dong, and Erkang Wang. Microfluidic Electrochemical Aptameric Assay Integrated On-Chip: A Potentially Convenient Sensing Platform for the Amplified and Multiplex Analysis of Small Molecules. Anal. Chem. 83 (2011). 1523-1529.

25. Ruigrok, Vincent J., Mark Levisson, Michel H. Eppink, Hauke Smidt, John Van Der Oost. "Alternative affinity tools: more attractive than antibodies?" Biochem. J. (2011). 436, 1-13.

26. Phillips, K. Scott, Quan Cheng. "Recent advances in surface plasmon resonance based techniques for bioanalysis." Anal. Bioanal. Chem. (2007) 387, 1831-1840.

27. J. Homola, S. S. Yee, G. Gauglitz. Surface plasmon resonance sensors: review 315

28. BI-2000 Manual v5.4 pg 1-3

29. van der Merwe, P. Anton. "Surface plasmon resonance." http://users.path.ox.ac.uk/ vdmerwe/internal/spr.pdf. 1-50.

30. Campbell, Charles T., Gibum Kim. "SPR microscopy and its applications to highthroughput analyses of bimolecular binding events and their kinetics." Biomaterials 28 (2007) 2380-2392.

31. Kodoyianni, Voula. "Label-free analysis of biomolecular interaction using SPR imaging." Biotechniques. (2011) Vol. 50 No. 1. 32-40.

32. Balamurugan, Subramanian, Anne Obubuafo, Steven A. Soper, David A. Spivak. "Surface immobilization methods for aptamer diagnostic applications." Anal. Bioanal. Chem. (2008) 390:1009-1021.

33. Chen, Y., Christian Wiesmann, Germaine Fuh, Bing Li, Hans W. Christinger, Patrick McKay, Abraham M. de Vos, Henry B. Lowman. "Selection and Analysis of an Optimized Anti-VEGF Antibody: Crystal Structure of an Affinity-matured Fab in Complex with Antigen." J. Mol. Biol. (1999) 293, 865-881.

34. Nonaka, Yoshihiko, Koji Sode, Kazunori Ikebukuro. "Scrrening and Improvement of an Anti-VEGF DNA Aptamer." Molecules. (2010), 15, 215-225.

35. de Belder, A. N., "Dextran." Amersham Biosciences. Edition AA. http://www-esbs.ustrasbg.fr/notesdecours/2eme-annee/purification_prot/PDF/18116612AA.pdf 
36. VEGF-A vascular endothelial growth factor A (Homo sapiens]. NCIB. 20-May-2012. http://www.ncbi.nlm.nih.gov/gene/7422

37. Recombinant Human VEGF 165. Syd Labs, Inc. Catalog No.: BP000027-GD1.

38. Rabbit Anti-VEGF-A Polyclonal Antibody, Unconjugated. Bioss Antibodies. Cat. Number: bs-4572R.

39. Ueda, Yasuji, Takehiro Yamagishi, Kazunori Samata. "A novel low molecular weight antagonist of vascular endothelial growth factor receptor binding: VGA1155." Molecular Cancer Therapeutics (2003) 2, 1105-1111.

40. Nagy, Janice A., Ann M. Dvorak, Harold F. Dvorak. "VEGF-A and the Induction of Pathological Angiogenesis." Pathology: Mechanisms of Disease. Vol. 2 (2007) 251275.

41. VEGF (human). PhosphoSite Plus. Cell Signaling Technology. 2003-2012. http://www.phosphosite.org/proteinAction.do?id=10608\&showAllSites=true

42. Livnah, O.; Bayer, E.; Wilchek, M.; Sussman, J. "Three-dimensional structures of avidin and the avidin-biotin complex". Proceedings of the National Academy of Sciences of the United States of America, (1993).90 (11): 5076-5080.

43. Nguyen (Win) Ly, Ph.D., Biosensing Instruments. SPR Technical Expert. Research and Development.

44. Protein Interaction Analysis. Bio Radiations. 2008 Bio-Rad Laboratories, Inc. 1-6. 45. O'Sullivan, Ciara K. Aptasensors- the future of biosensing? Anal. Bioanal. Chem. (2002). 372: 44-48. 


\section{APPENDIX}

\section{A. Calculations}

\begin{tabular}{|c|c|c|}
\hline \multicolumn{3}{|c|}{ for high affinity molecules: $10-100 \mathrm{nM}$} \\
\hline optimal: 50nM & & \\
\hline VEGF MW & 19200 & $\mathrm{~g} /$ mole \\
\hline Antibody MW & 45000 & $\mathrm{~g} / \mathrm{mole}$ \\
\hline Aptamer MW & 8758.7 & $\mathrm{~g} /$ mole \\
\hline $\mathrm{NaOH} \mathrm{MW}$ & 40 & $\mathrm{~g} /$ mole \\
\hline $\mathrm{HCl} \mathrm{MW}$ & 36.46 & $\mathrm{~g} / \mathrm{mole}$ \\
\hline
\end{tabular}

Molecular weights for all

\begin{tabular}{|c|c|c|}
\hline $\mathrm{NaOH}$ & & \\
\hline & 20 & $\mathrm{mM}$ \\
\hline & $2.00 \mathrm{E}-02$ & moles/liter \\
\hline & 0.0001 & $100 \mathrm{uL}$ in liters \\
\hline & $2.00 \mathrm{E}-06$ & moles of $\mathrm{NaOH}$ \\
\hline & $8.00 \mathrm{E}-05$ & grams of $\mathrm{NaOH}$ \\
\hline & 80 & ug to add to $100 \mathrm{uL}$ \\
\hline
\end{tabular}

Calculations for the $\mathrm{NaOH}$ solution

\begin{tabular}{|c|c|c|c|c|c|}
\hline \multirow[t]{2}{*}{ VEGF } & & & Check & \multirow[b]{3}{*}{ g/liter } & \\
\hline & 150 & $\mathrm{nM}$ & & & \\
\hline & $1.50 \mathrm{E}-07$ & Moles/liter & 0.00288 & & \\
\hline & 0.0001 & $100 \mathrm{uL}$ in liters & $2.88 \mathrm{E}-09$ & g/uL & \\
\hline & $1.5 \mathrm{E}-11$ & moles of VEGF & 100 & uL & \\
\hline & $2.88 \mathrm{E}-07$ & grams VEGF & $2.88 \mathrm{E}-07$ & \multicolumn{2}{|c|}{$\mathrm{g}$ to add to $100 \mathrm{uL}$ of solution } \\
\hline & 0.288 & ug to add to $100 \mathrm{uL}$ & & & \\
\hline & & & & & \\
\hline total VEGF & 10 & ugram & & & \\
\hline \multirow[t]{2}{*}{ solution to add } & 3472.222222 & uL & & & \\
\hline & 3.472222222 & $\mathrm{~mL}$ & & & \\
\hline
\end{tabular}

Calculation for the 150nM VEGF solution

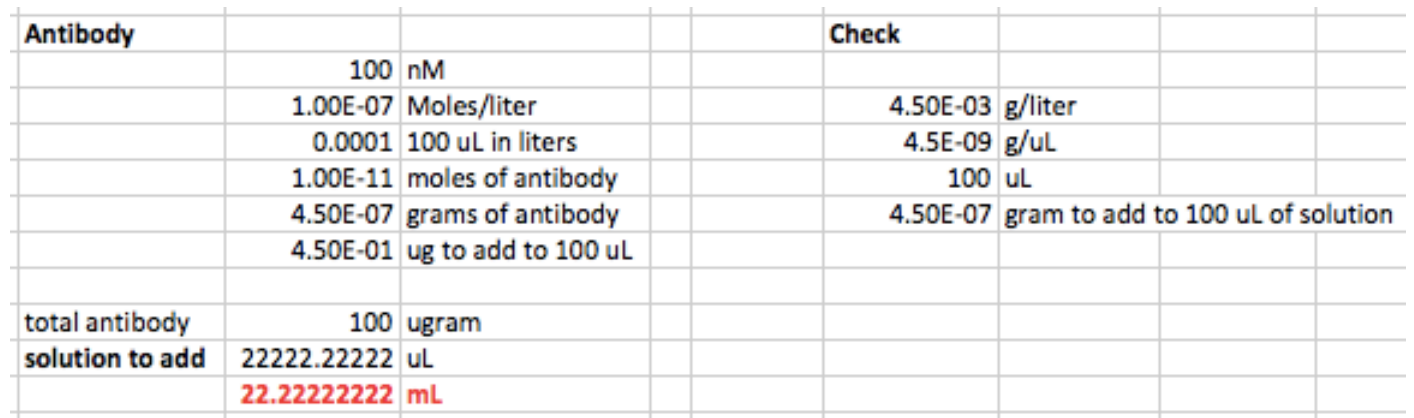

Calculation for the $100 \mathrm{nM}$ antibody solution

\begin{tabular}{|c|c|c|c|c|c|c|c|c|c|}
\hline \multicolumn{10}{|l|}{ Aptamer } \\
\hline & Current solution & c1 & & & & c2 & & v2 & \\
\hline & $43.79 \mathrm{ug} / \mathrm{uL}$ & 43.79 & & v1 & $=$ & 0.00087587 & ug/ul & 9999.20079 & uL \\
\hline & $937.46 \mathrm{uL}$ & & ug/ul & 0.2 & uL & & & 9.99920079 & $\mathrm{~mL}$ \\
\hline & & & & & & & & 9.79920079 & $\mathrm{~mL}$ \\
\hline & & & & & Volume & dd to $v 1$ for $c$ ? & & 9799.20079 & \\
\hline & & & & & & & & 8819.20079 & \\
\hline
\end{tabular}

Calculation for the $100 \mathrm{nM}$ aptamer solution 


\begin{tabular}{|c|c|c|c|c|c|c|c|}
\hline 50 & $\mathrm{nM}$ & & & & & & \\
\hline c1 & & v1 & $=$ & c2 & & $\mathrm{v} 2$ & \\
\hline 100 & $\mathrm{nM}$ & 1 & $\mathrm{~mL}$ & 40 & $\mathrm{nM}$ & 2.5 & $\mathrm{~mL}$ \\
\hline & & & & \multicolumn{2}{|c|}{ Volume to add to $\mathrm{v} 1$ for $\mathrm{c} 2$} & 1.5 & ml \\
\hline & & & & & & 1500 & \\
\hline
\end{tabular}

Calculation for the $50 \mathrm{nM}$ solutions

\begin{tabular}{|c|c|c|c|c|c|c|c|}
\hline 25 & $\mathrm{nM}$ & & & & & & \\
\hline $\mathrm{c} 1$ & & v1 & $=$ & $\mathrm{c} 2$ & & v2 & \\
\hline 100 & $\mathrm{nM}$ & 1 & $\mathrm{~mL}$ & 25 & $\mathrm{nM}$ & 4 & $\mathrm{~mL}$ \\
\hline & & & & \multicolumn{2}{|c|}{ Volume to add to v1 for $\mathrm{c} 2$} & 3 & $\mathrm{~mL}$ \\
\hline
\end{tabular}

Calculation for the 25nM solutions

\begin{tabular}{|l|c|c|c|c|c|c|}
\hline HCl & & & & & & \\
\hline
\end{tabular}

Calculation for the $\mathrm{HCl}$ solution

\begin{tabular}{|c|c|c|c|c|}
\hline Volume init & \multirow{2}{*}{$\begin{array}{r}0.0036 \\
2.00 E-07\end{array}$} & \multirow{2}{*}{\begin{tabular}{|l} 
liters \\
moles/liter
\end{tabular}} & \multirow{2}{*}{ or $4 \mathrm{~mL}$} & \multirow[t]{2}{*}{$\mathrm{pH}=7.3$} \\
\hline [OH-]init & & & & \\
\hline pOH init & 6.70 & & & \\
\hline moles $\mathrm{OH}-$ & $7.18 \mathrm{E}-10$ & moles $\mathrm{OH}-$ & & \\
\hline Volume added & 1.8 & ul & & \\
\hline (NaOH soln) & 0.0000018 & liters & & \\
\hline [NaOH] added & 0.02 & \multicolumn{2}{|c|}{ moles/liter } & \\
\hline moles added & $3.60 \mathrm{E}-08$ & moles $\mathrm{OH}$ - & & \\
\hline [OH-]new & $1.01944 \mathrm{E}-05$ & moles/liter & & \\
\hline $\mathrm{pOH}$ & 4.991637104 & & & \\
\hline $\mathrm{pH}$ & 9.008362896 & & & final $\mathrm{ph}=8.6$ \\
\hline
\end{tabular}

Calculations for attempting to change the $\mathrm{pH}$ to 8.6, the PBS buffer resisted the change of $\mathrm{pH}$ so $\mathrm{pH}$ was changed using a $\mathrm{pH}$ probe 


\section{B. SPR Ethanol RaW Data}

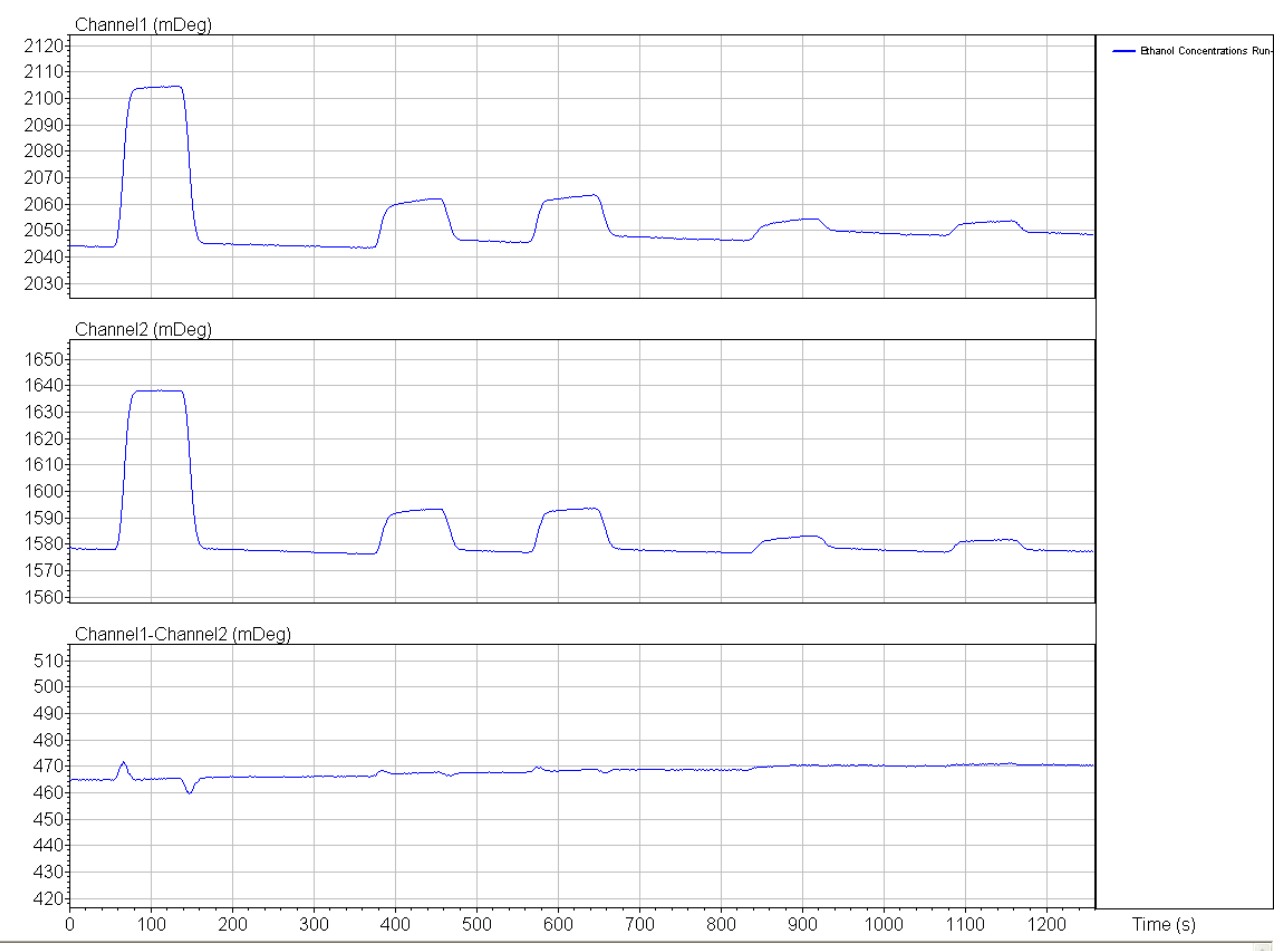

Overall ethanol dilution experiment

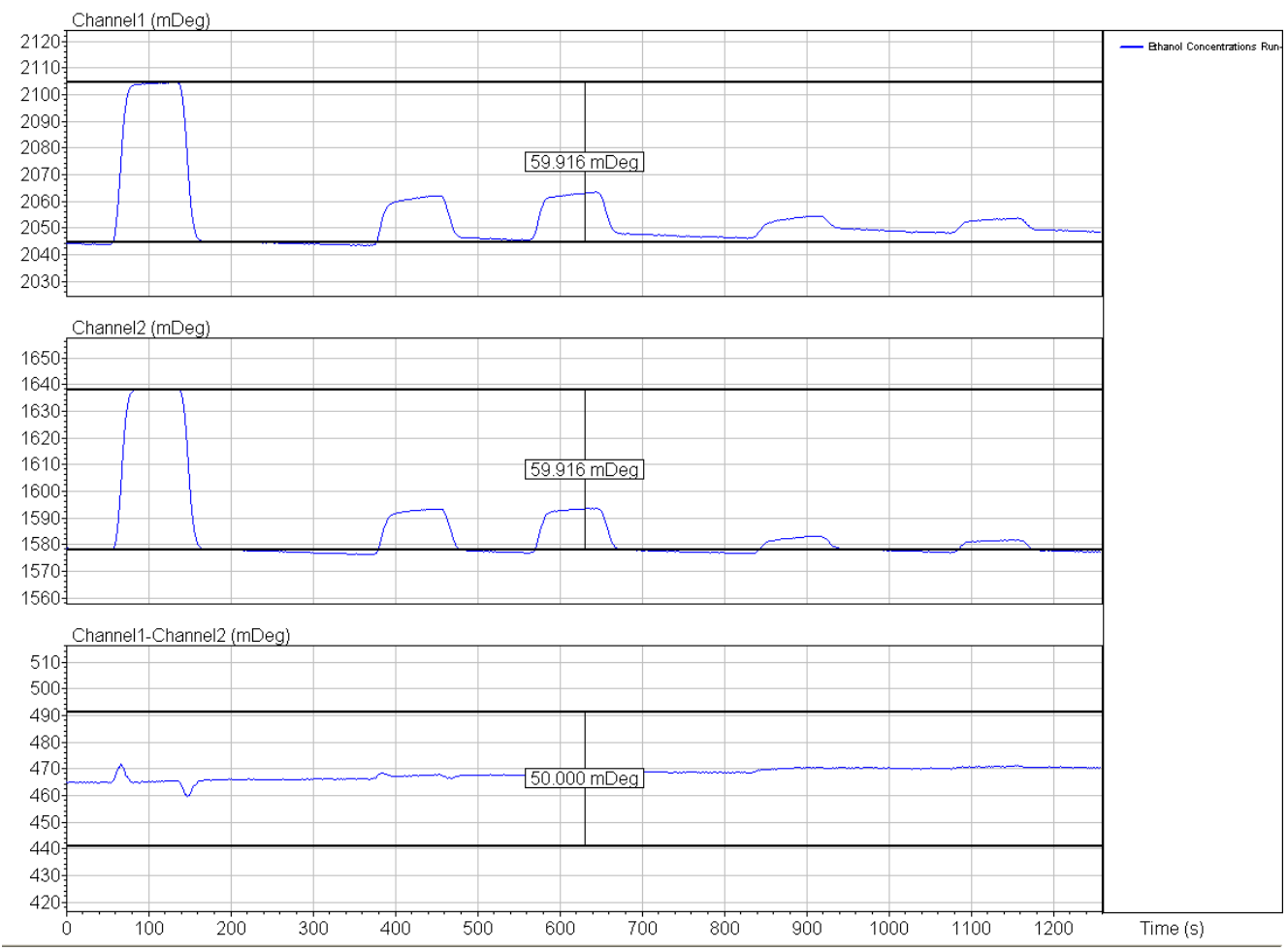

mDeg of the $1 \%$ ethanol solution 


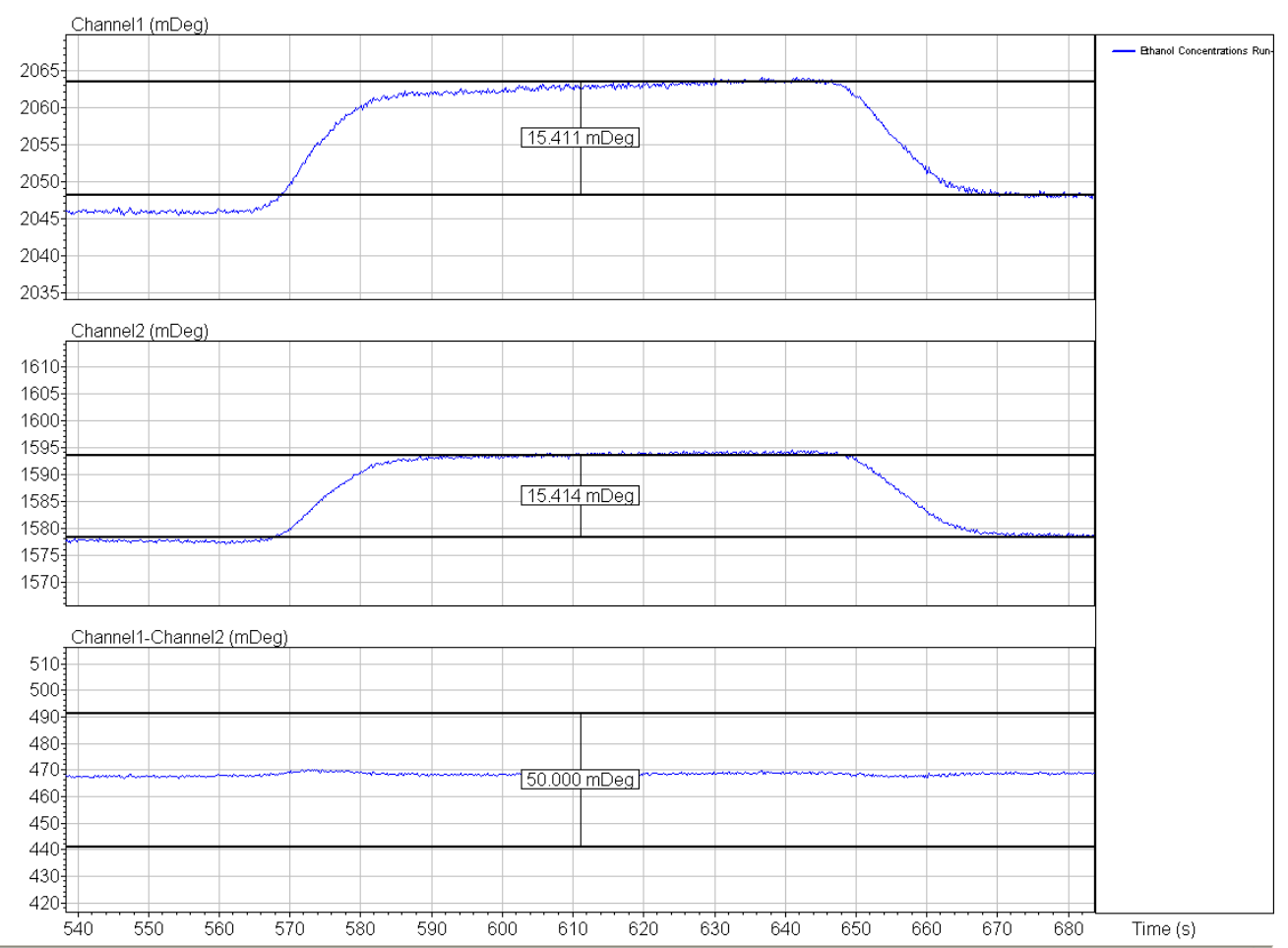

mDeg of the $0.25 \%$ ethanol solution
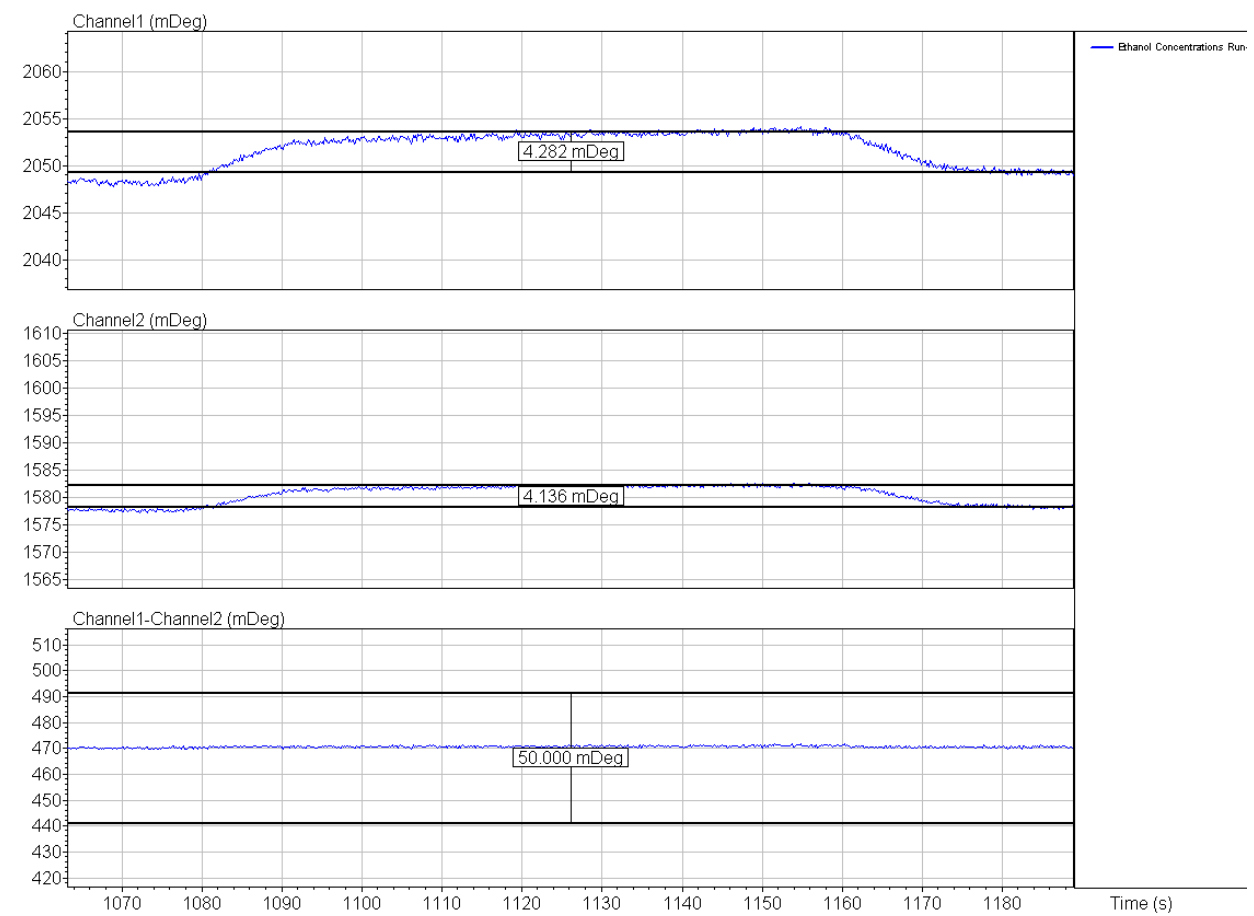

\section{mDeg of the $0.0625 \%$ ethanol solution}




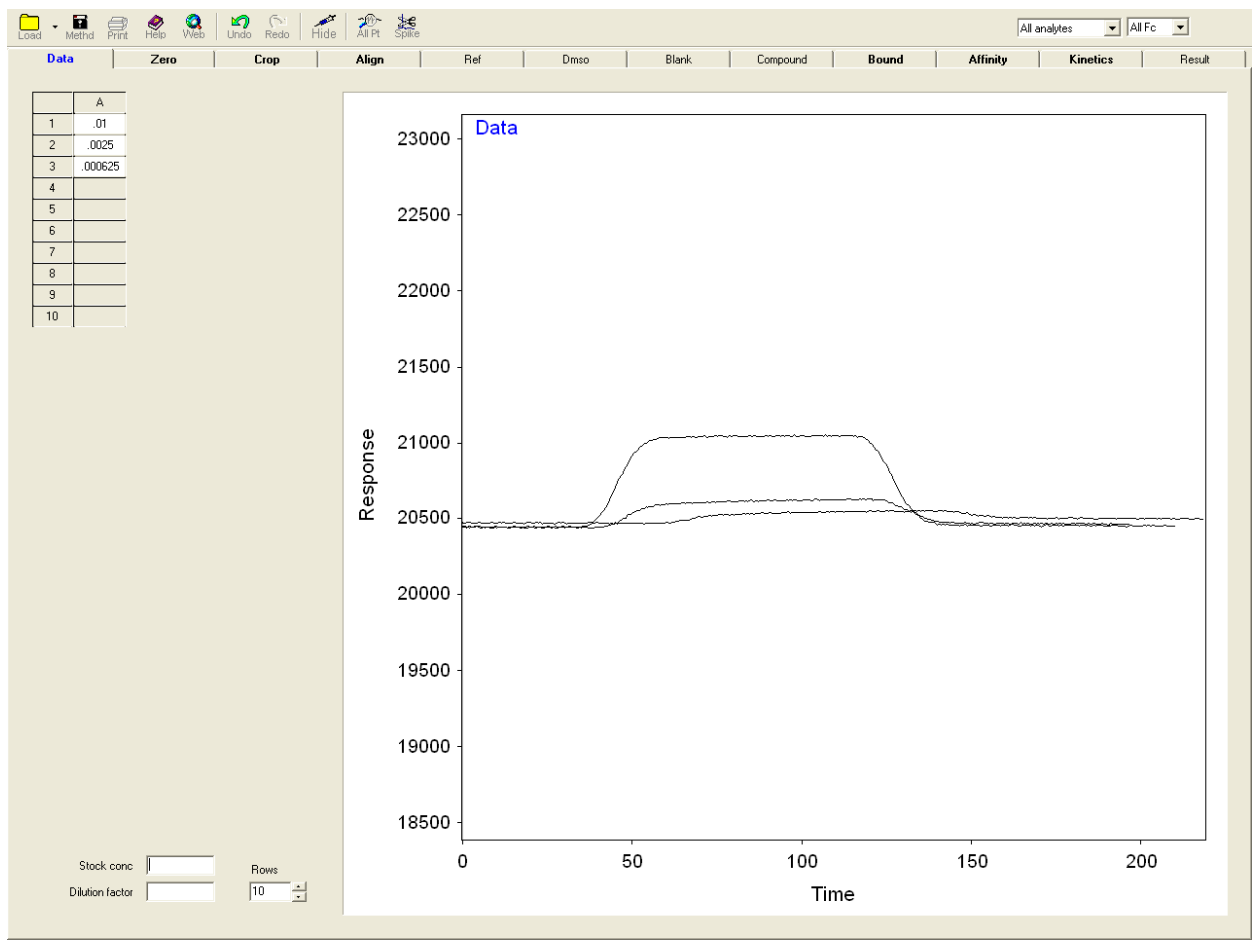

\section{Ethanol curves input into the BI-Analysis program}

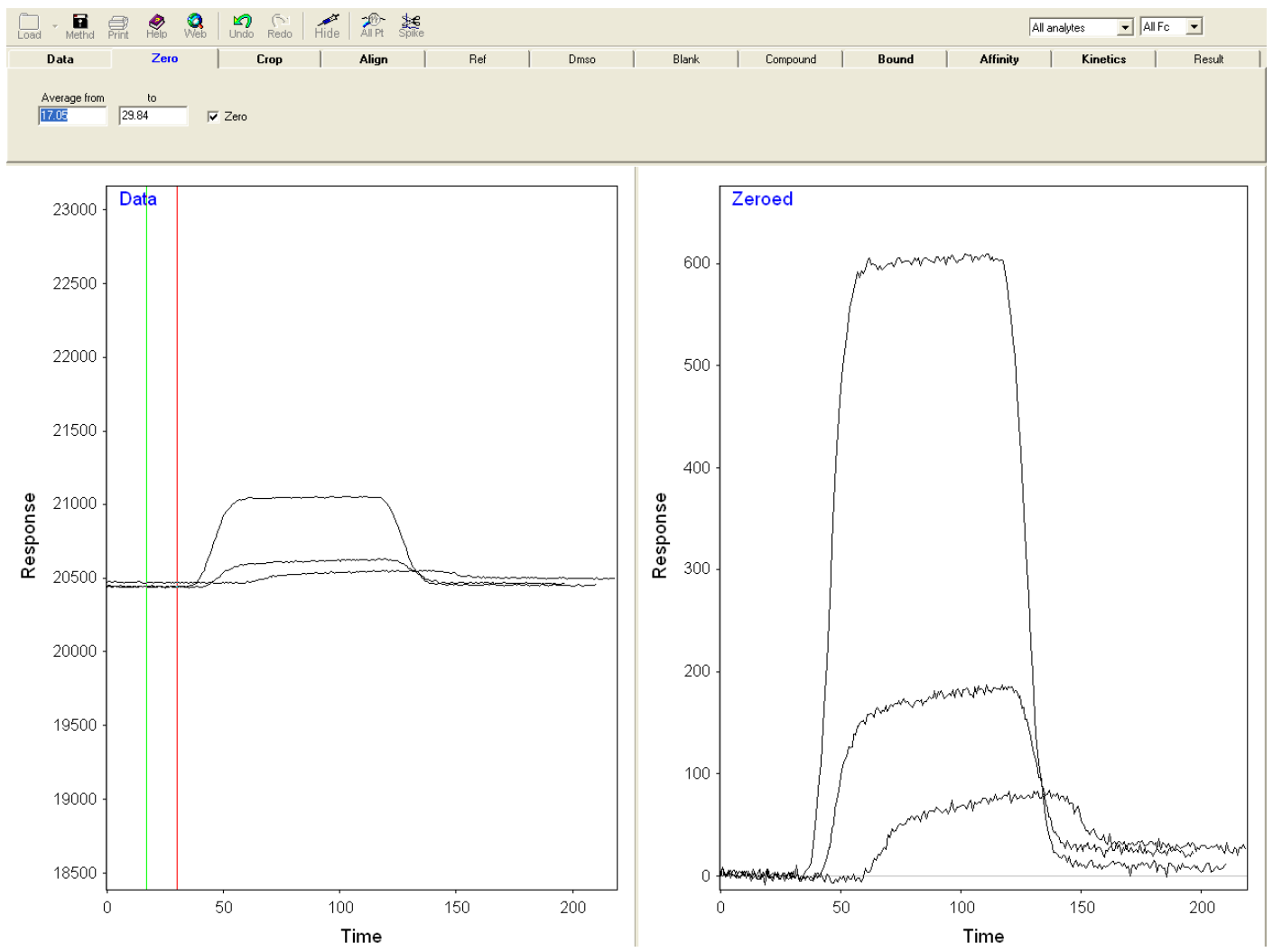

\section{Zeroing page of the ethanol analysis}



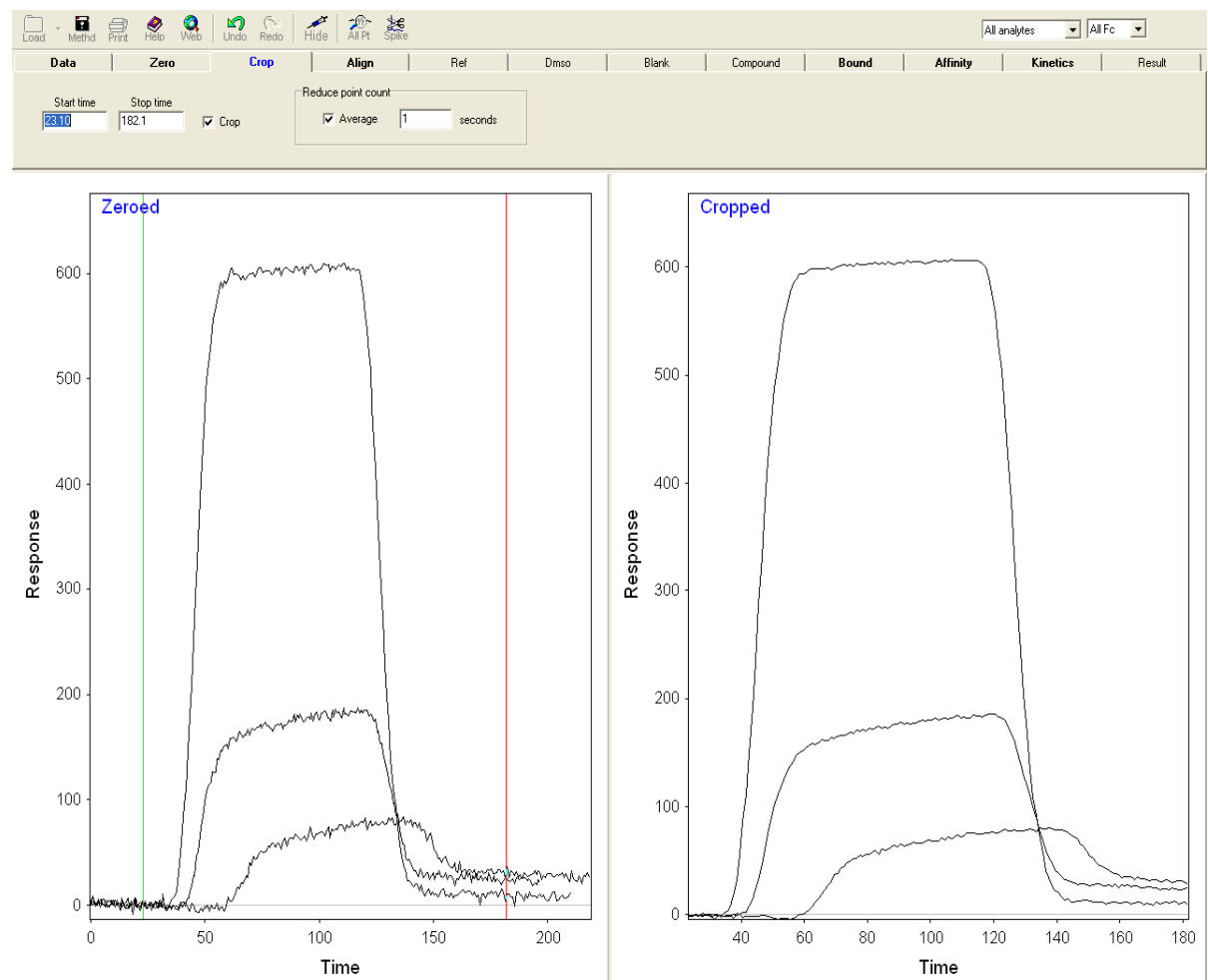

\section{Cropping page of the ethanol analysis}
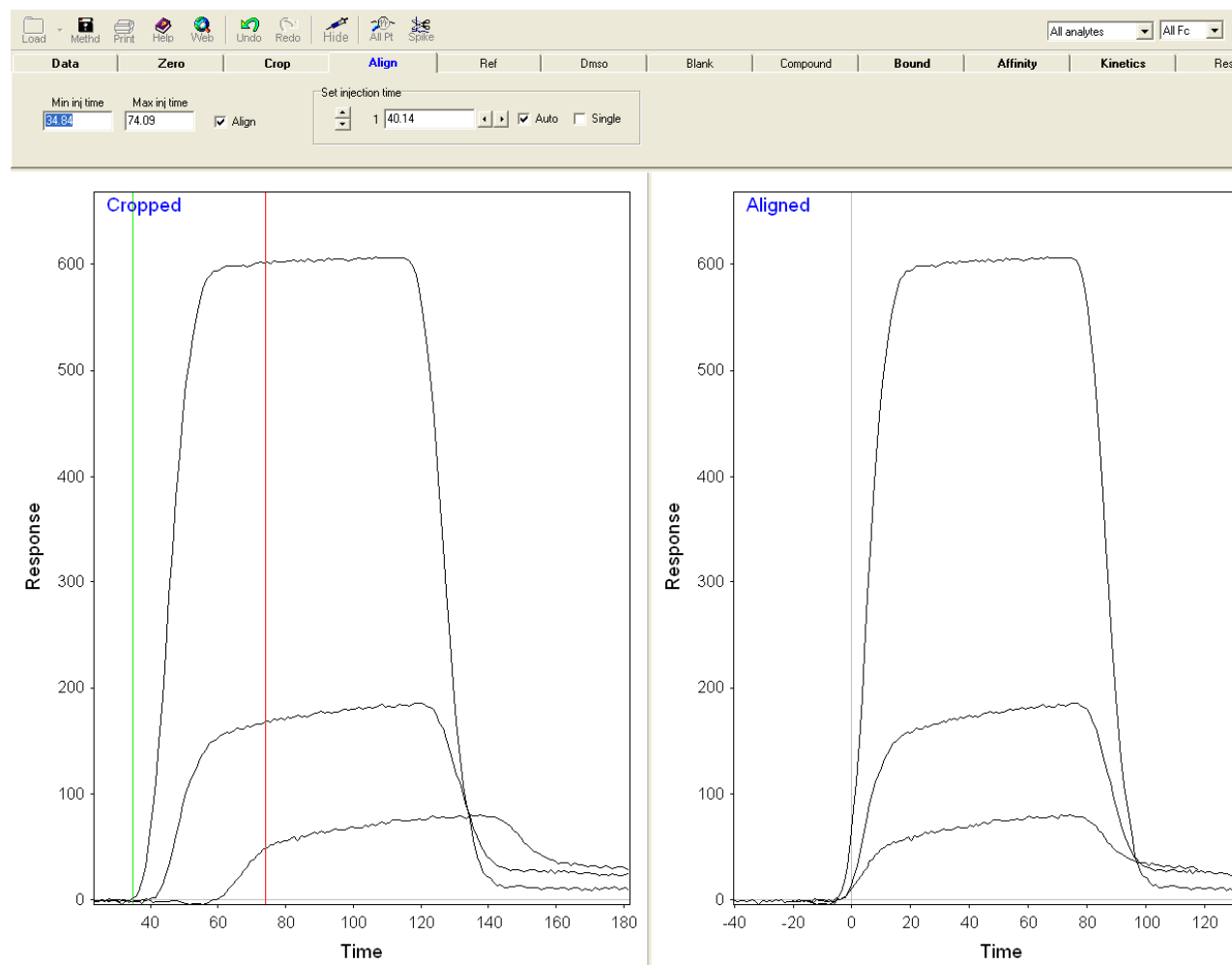

Data | Zero | Crop Align | Ref | Dmso $\mid$ Blank $\mid$ Compound $\mid$ Bound $\mid$ Affinity $\mid$ Kinetics | Result

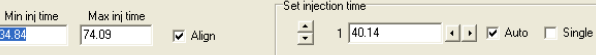

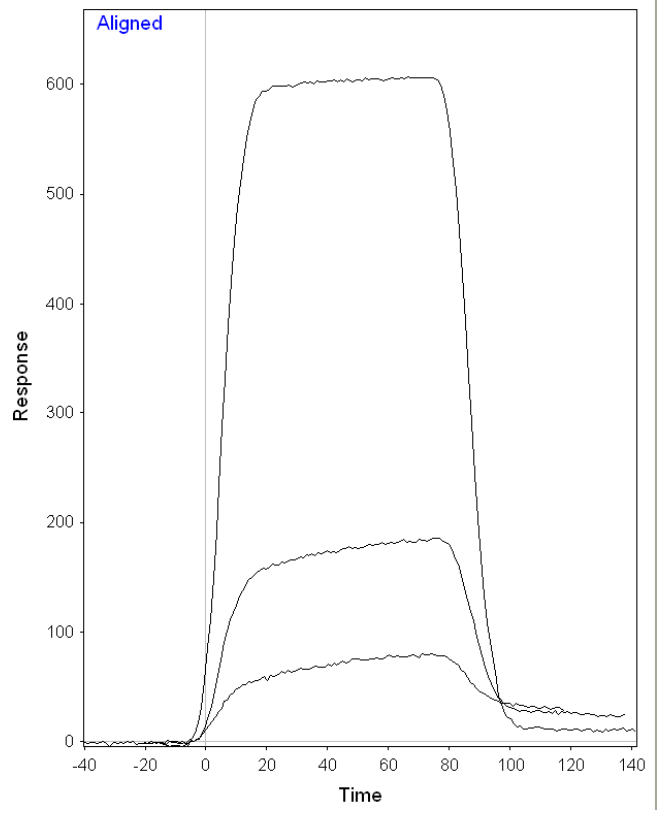

Alignment page of the ethanol analysis 


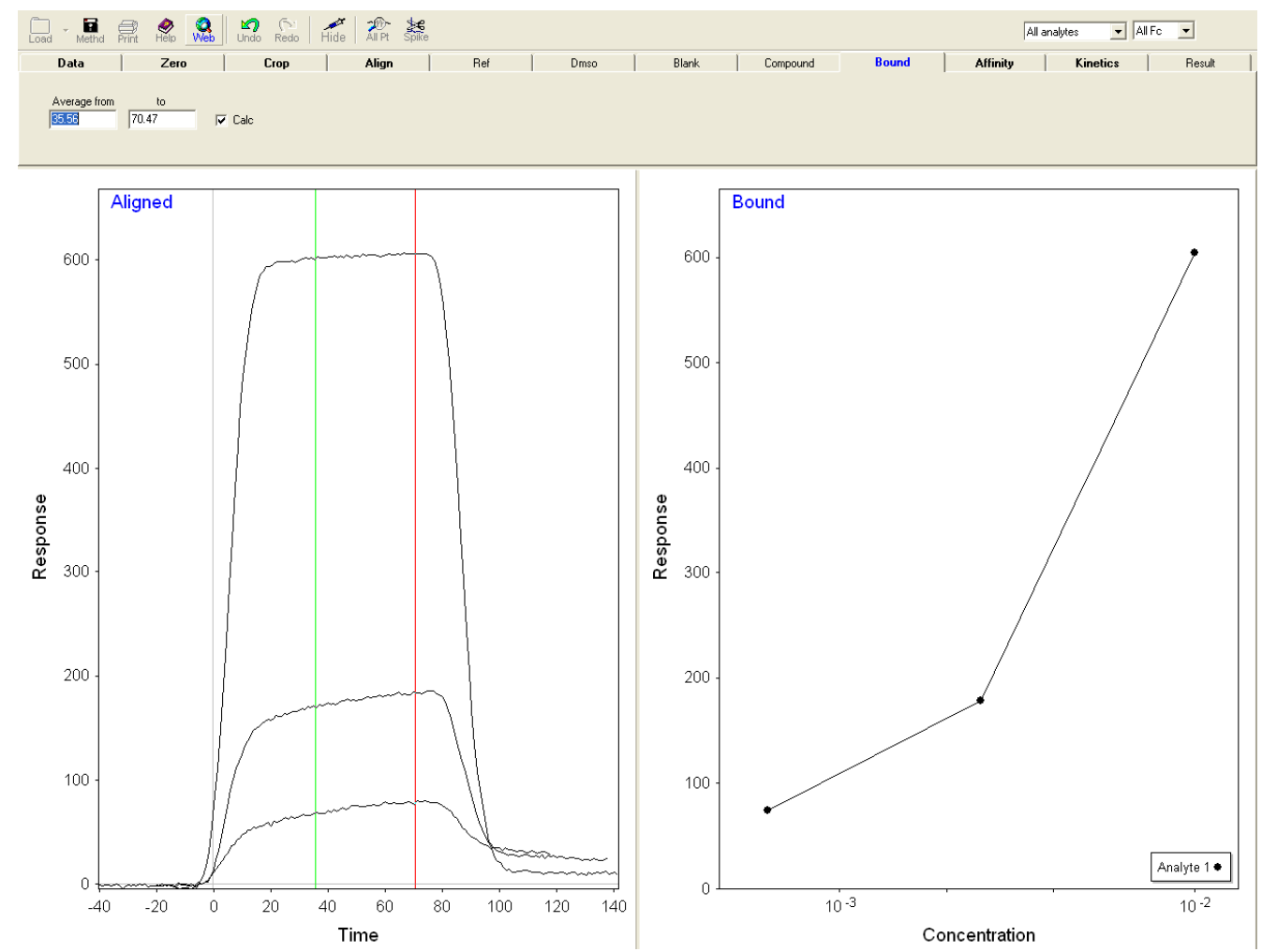

Analyte bound page of the ethanol analysis

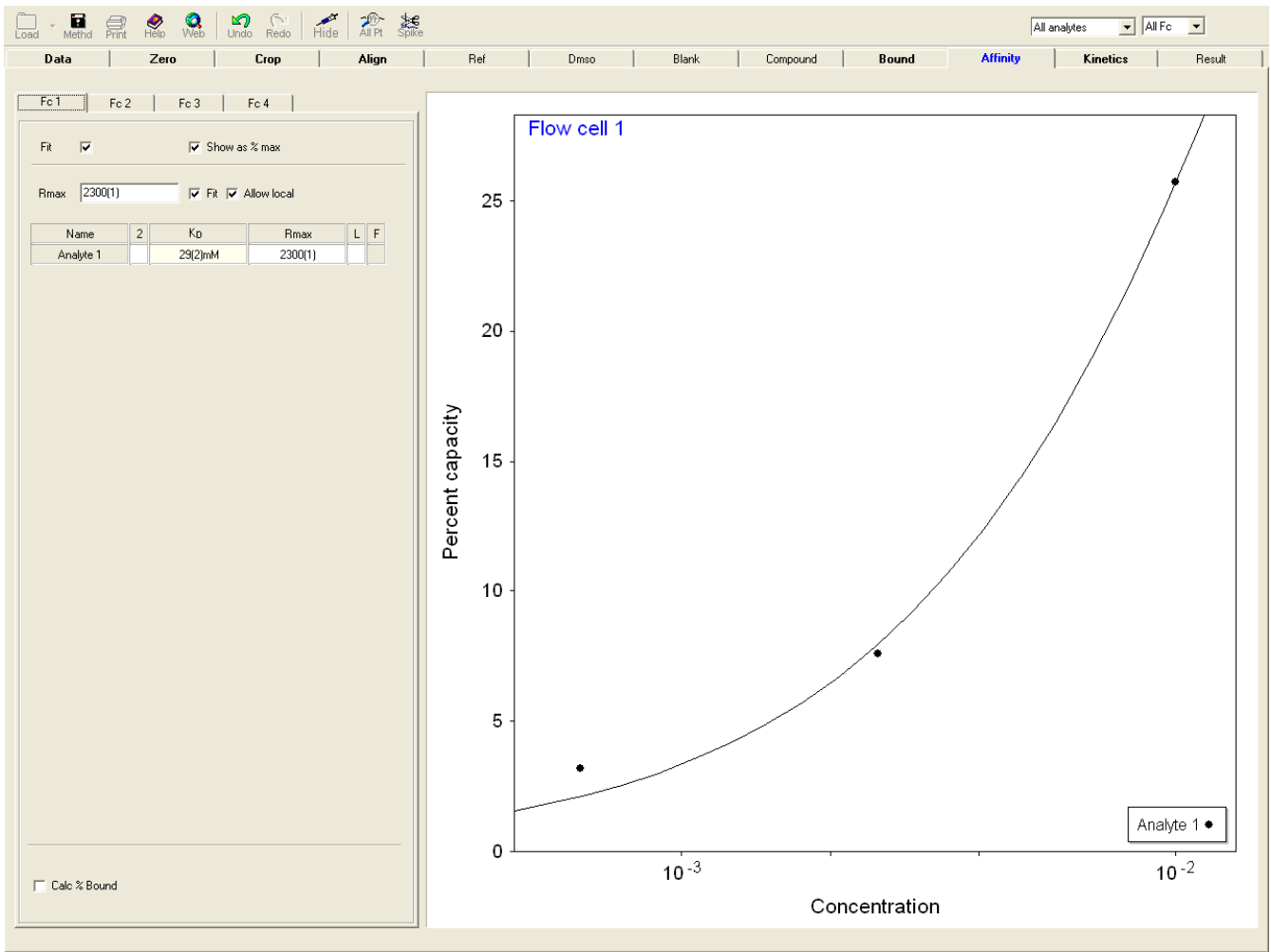

Affinity page of the ethanol analysis 


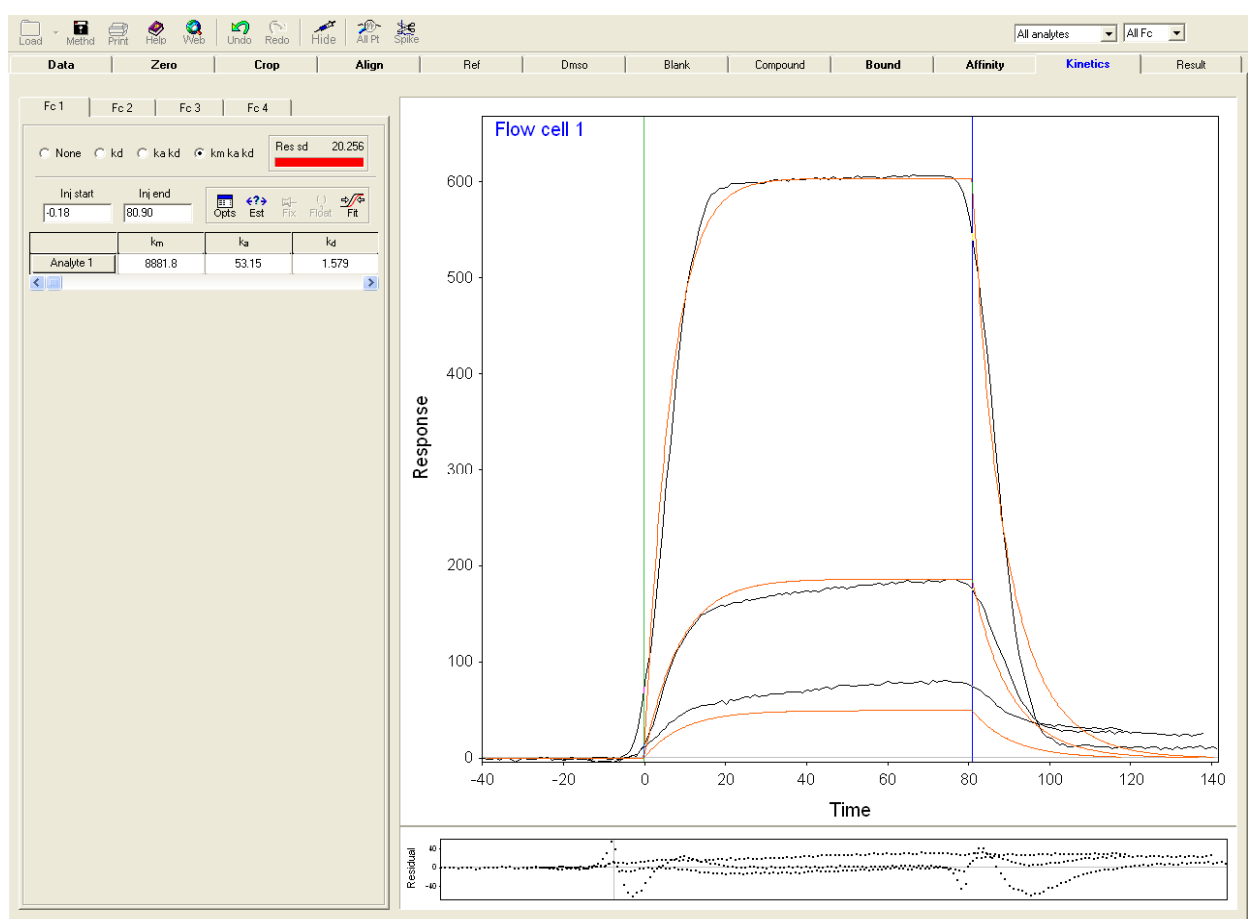

Kinetics page of the ethanol analysis page 1

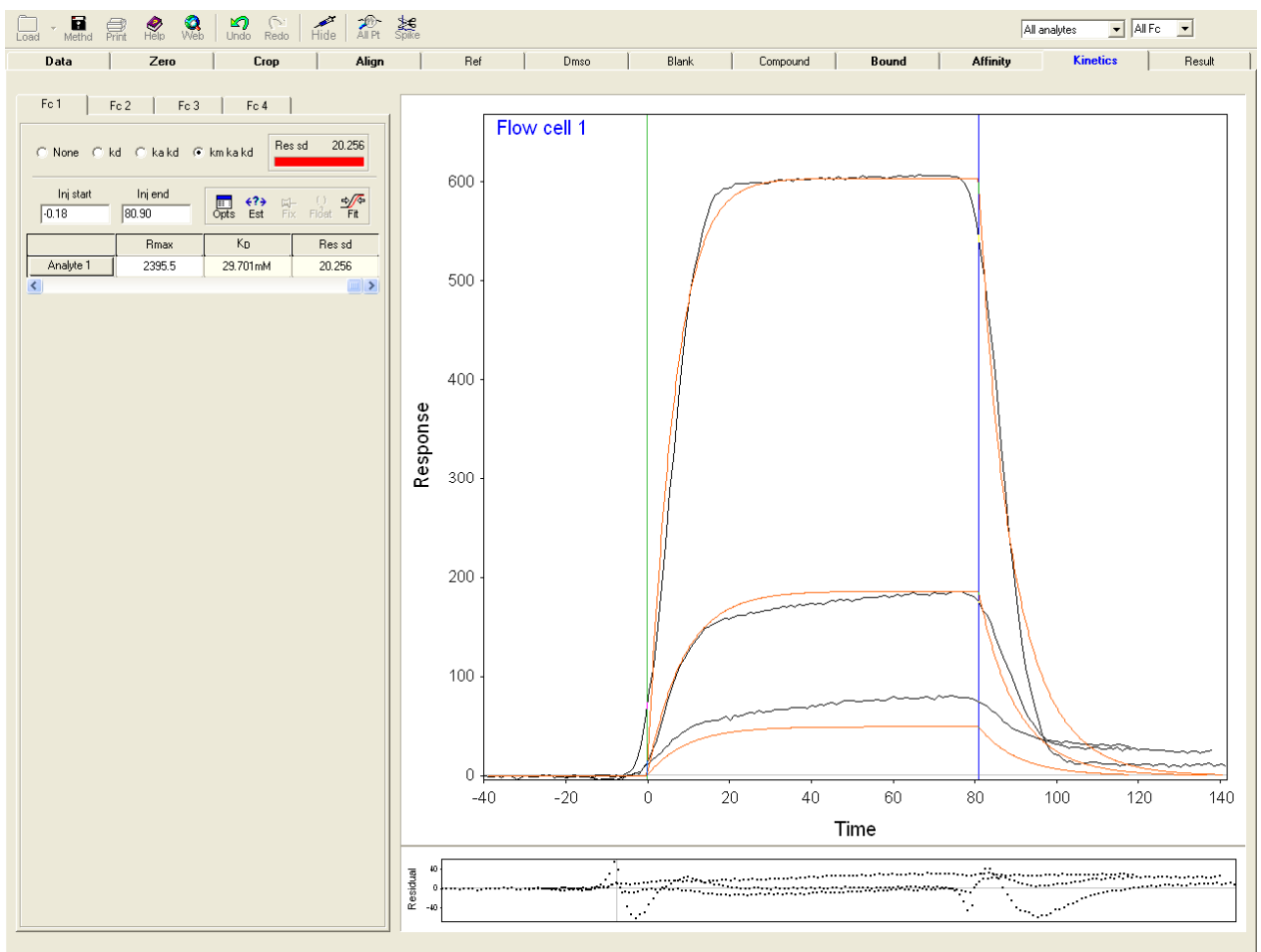

Kinetics page of the ethanol analysis page 2 


\section{SPR Run 1 RAW DATA}
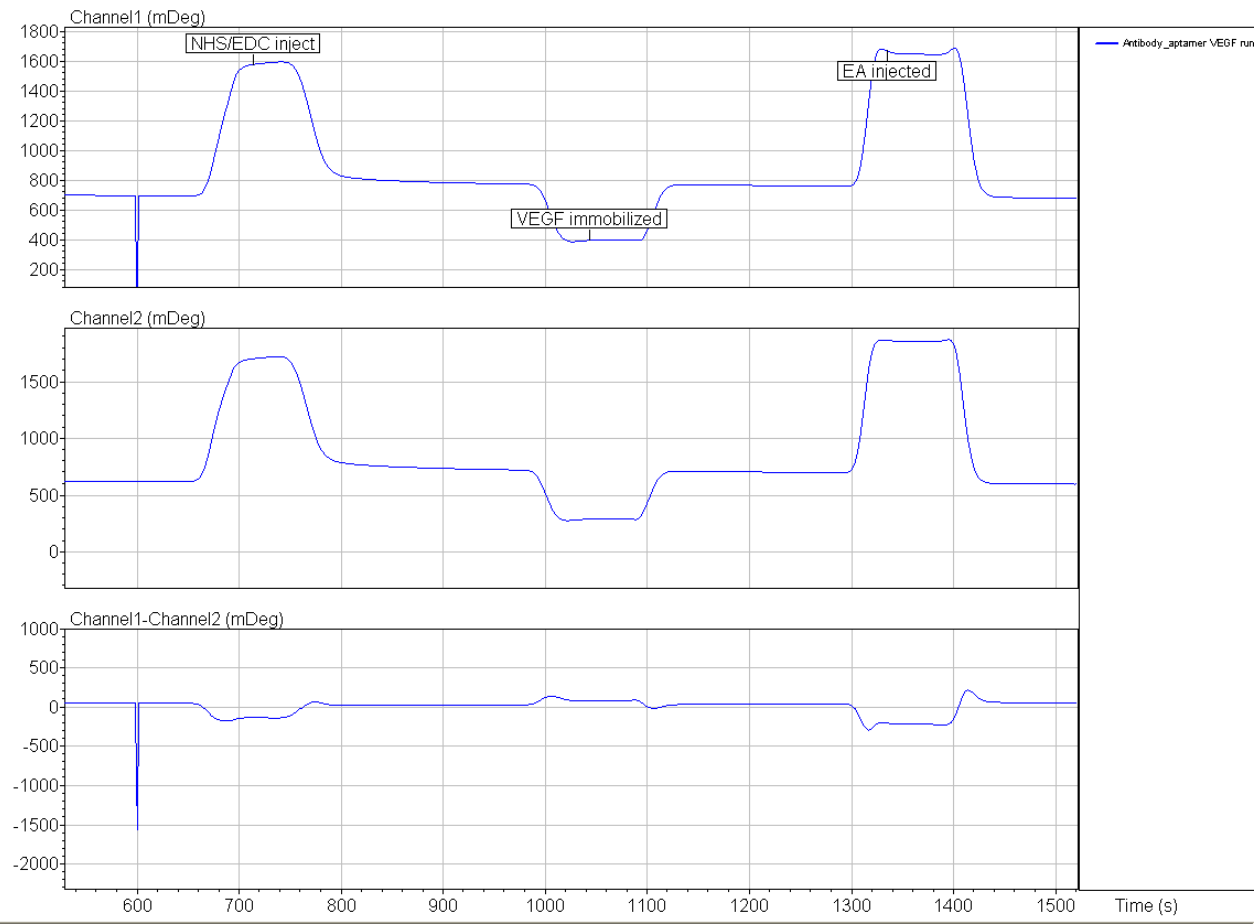

Activation of CM-Dextran and attempted immobilization of VEGF-A
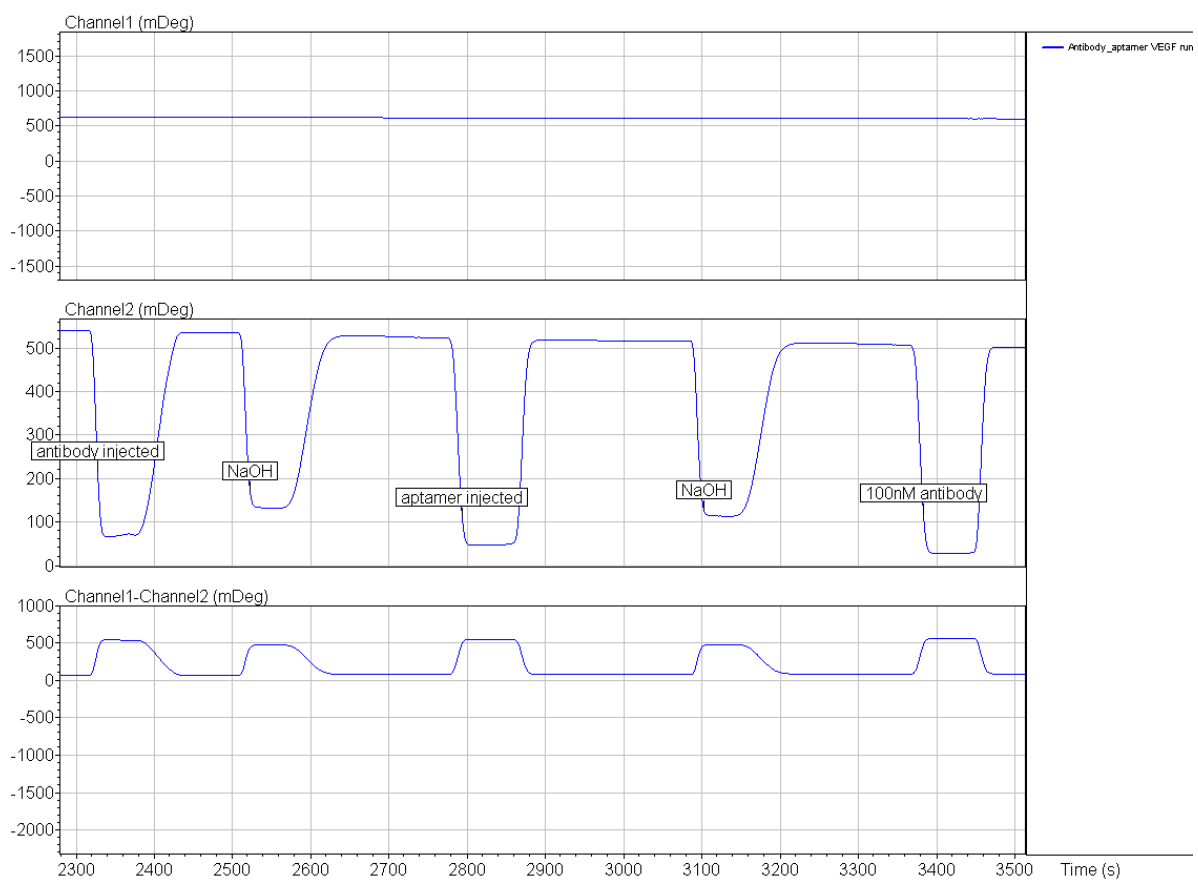

Overall injections of sample for Run 1 page 1 


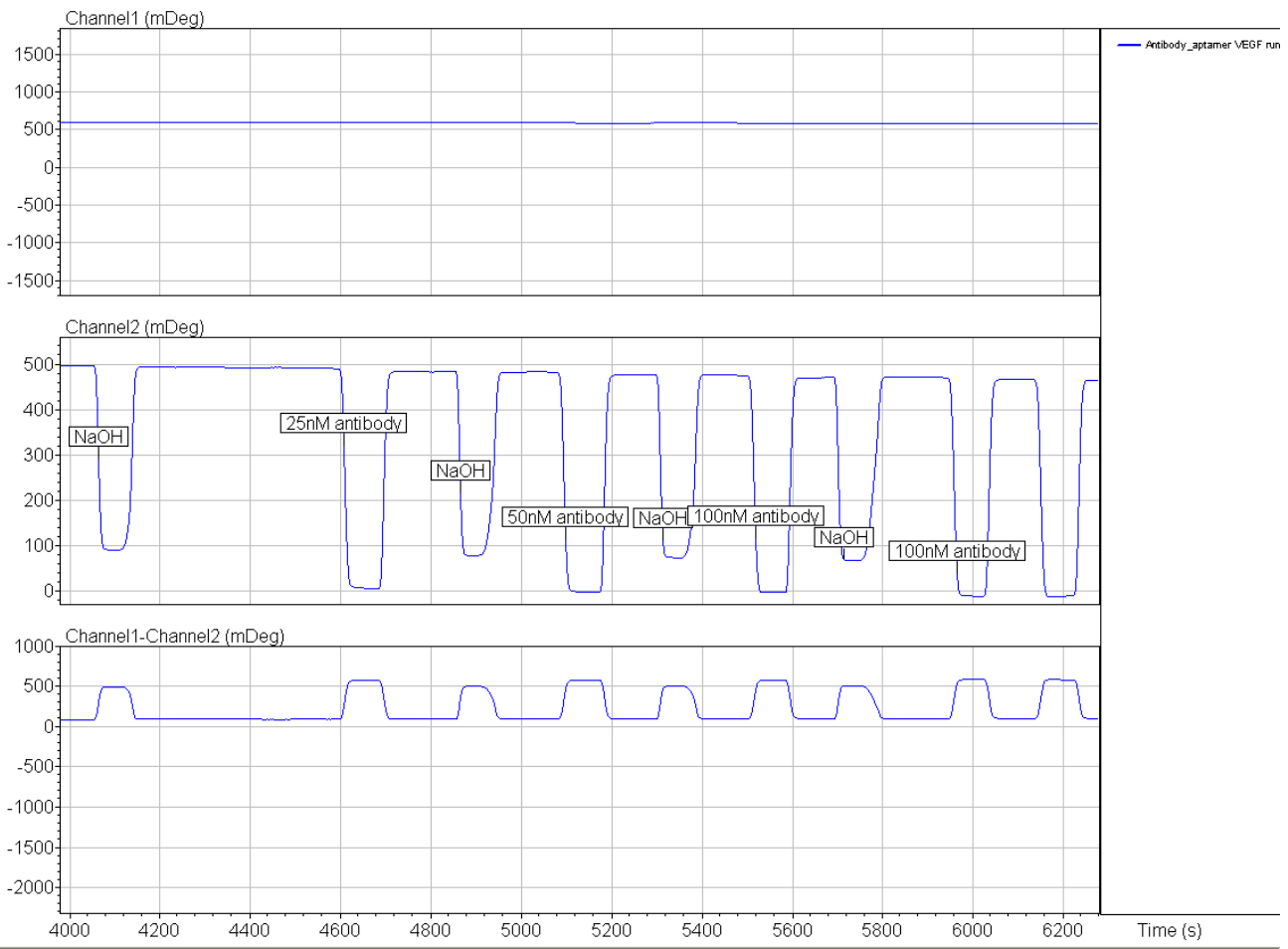

Overall injections of sample for Run 1 page 2 


\section{SPR RUN 2 RAW DATA}

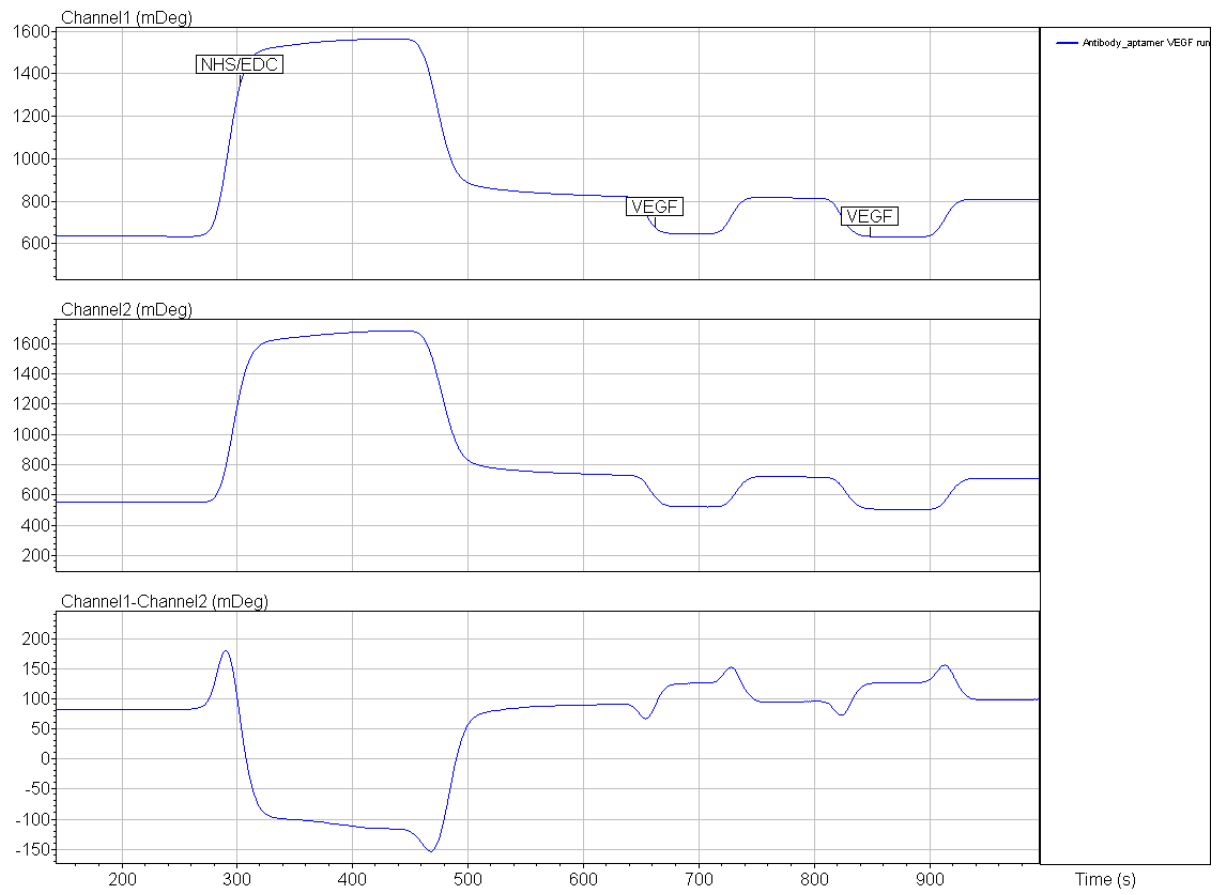

Activation of CM-Dextran surface and attempted immobilization of VFCF-A 


\section{E. SPR Run 3 RaW Data}

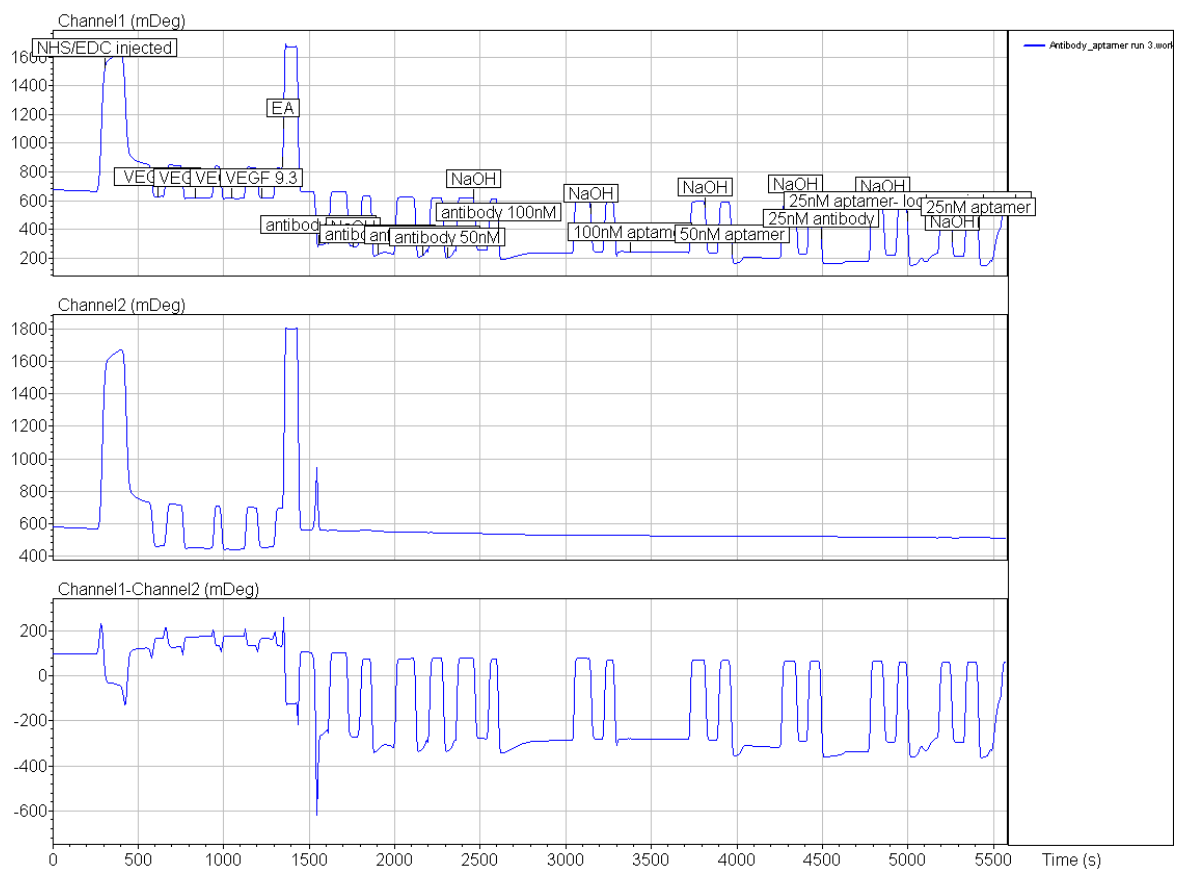

\section{Overall SPR Run 3}

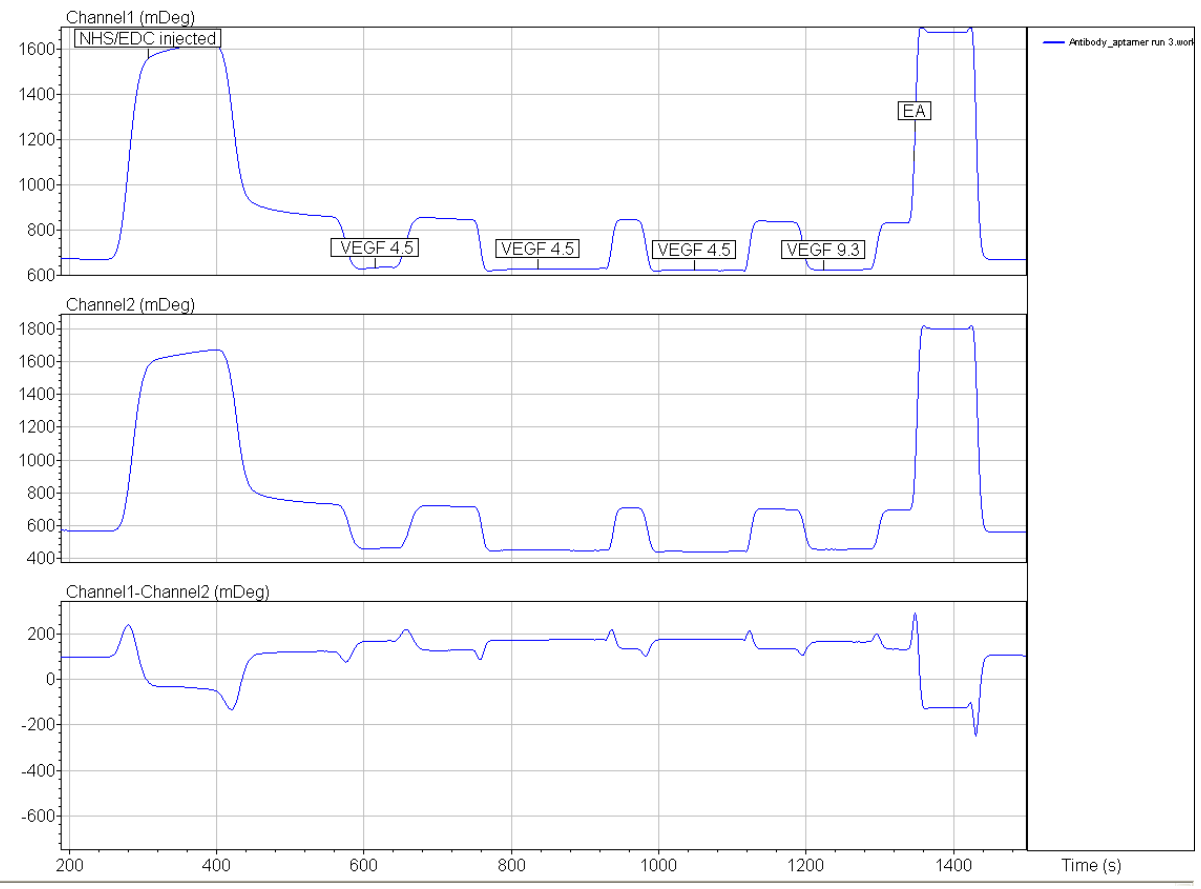

Immobilization of VEGF-A 


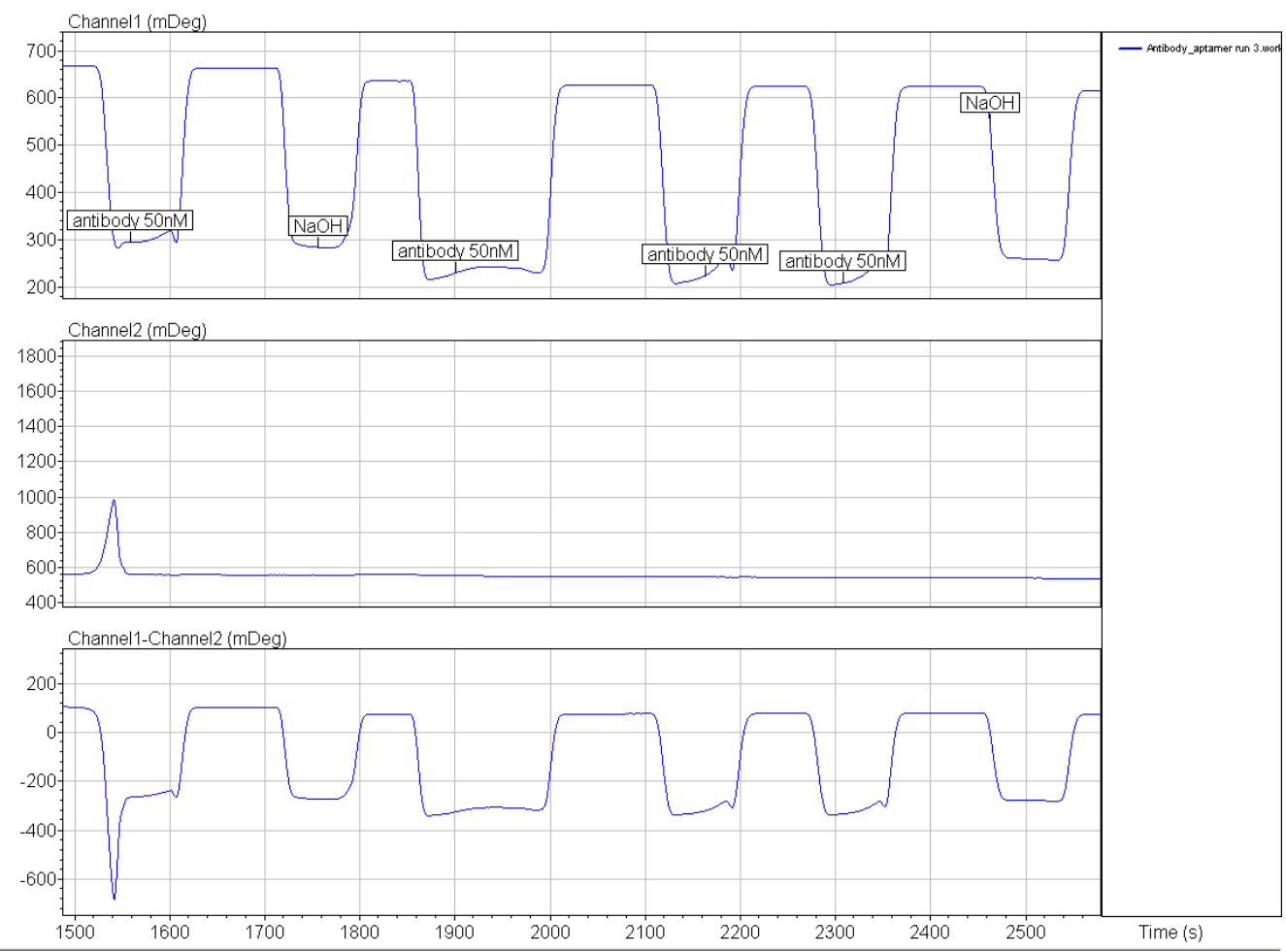

Run 3 injections page 1
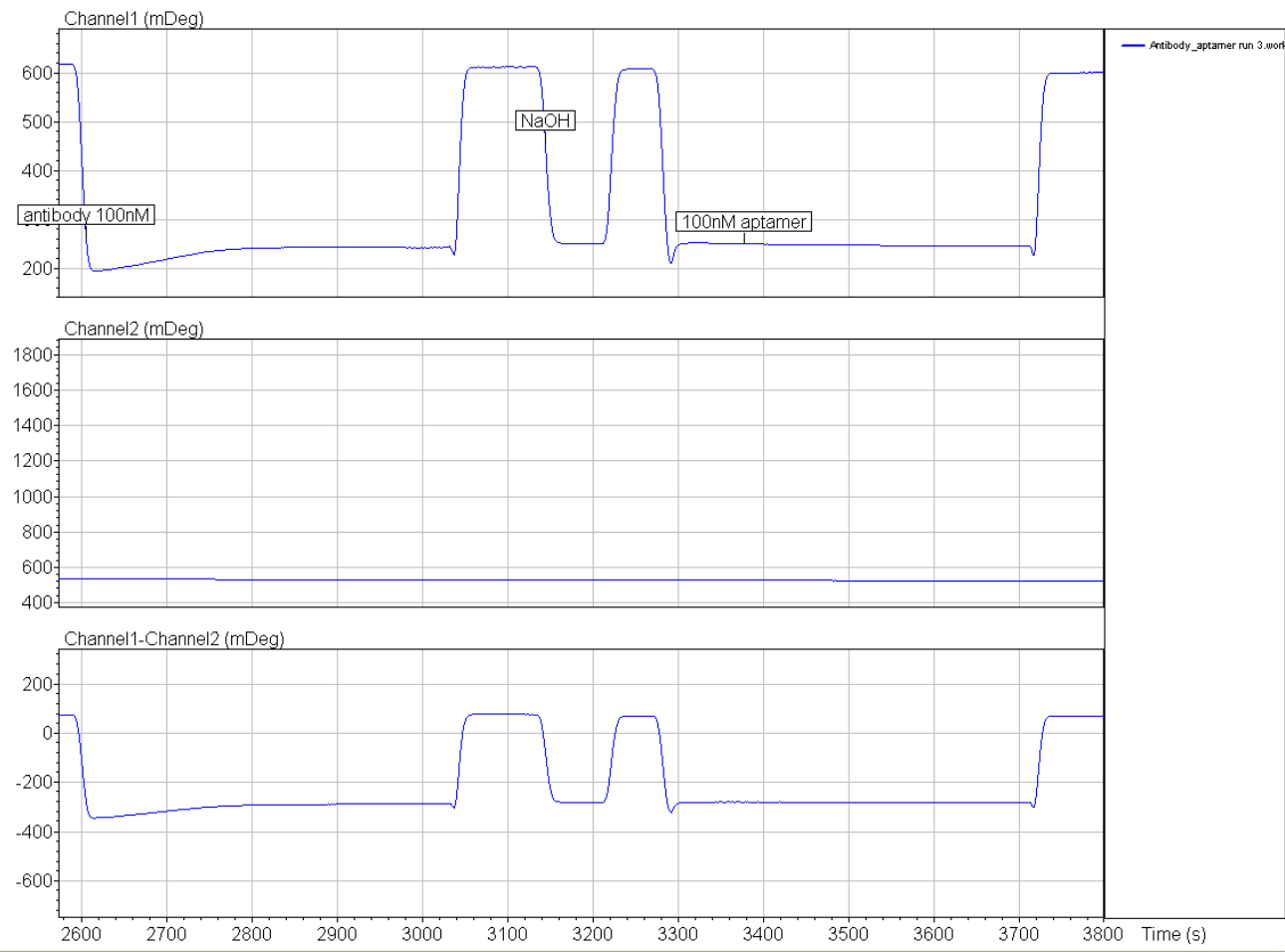

Run 3 injections page 2 

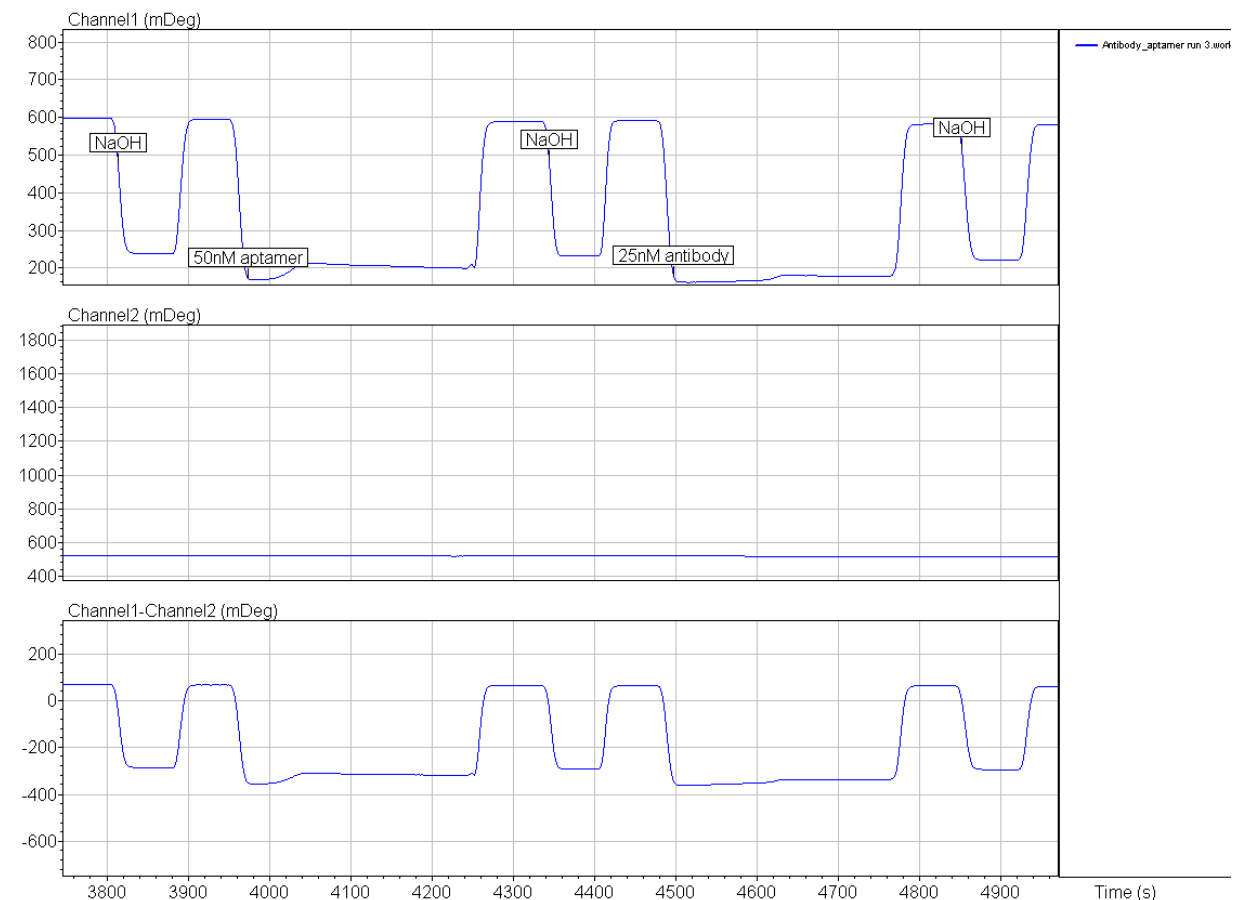

Run 3 injections page 3

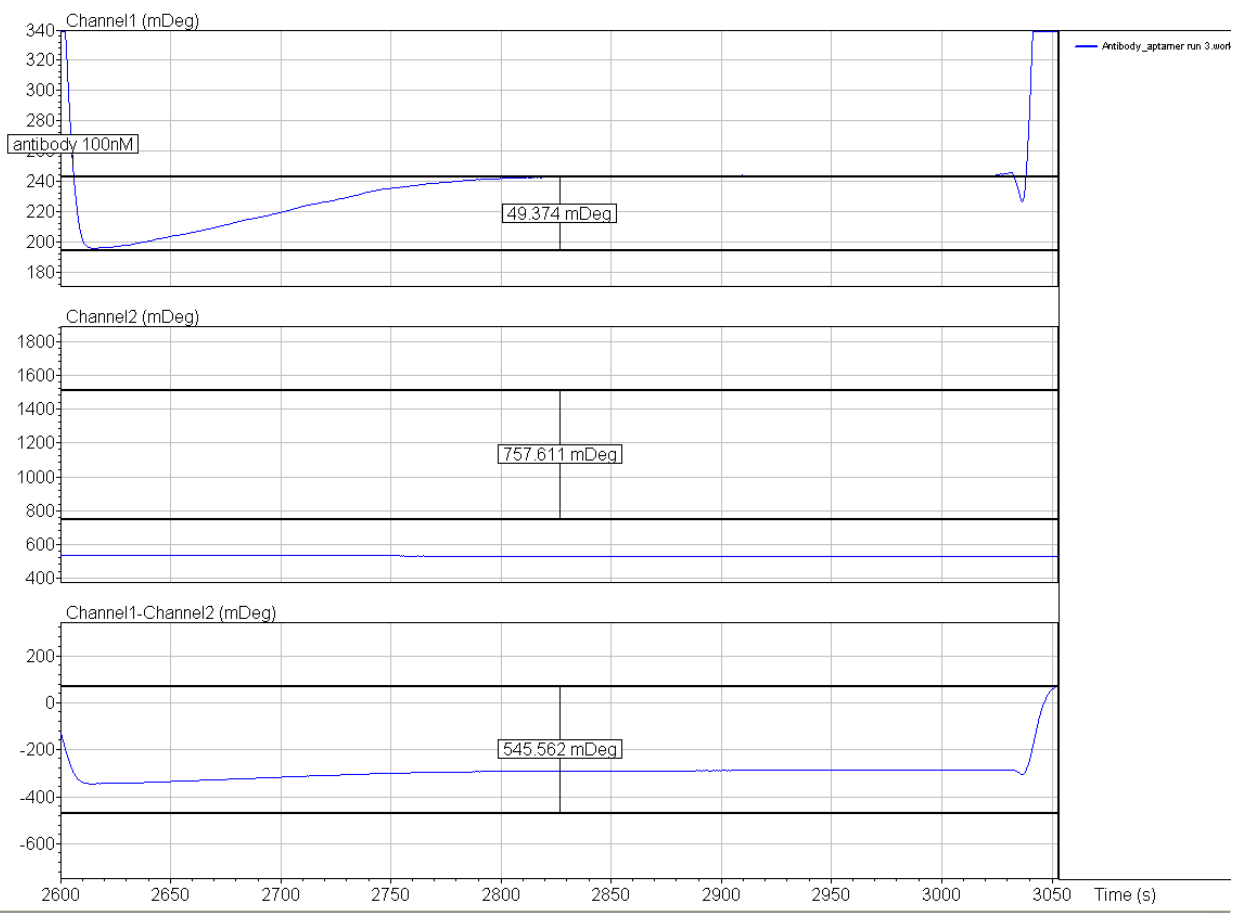

100nM antibody qualitative analysis 

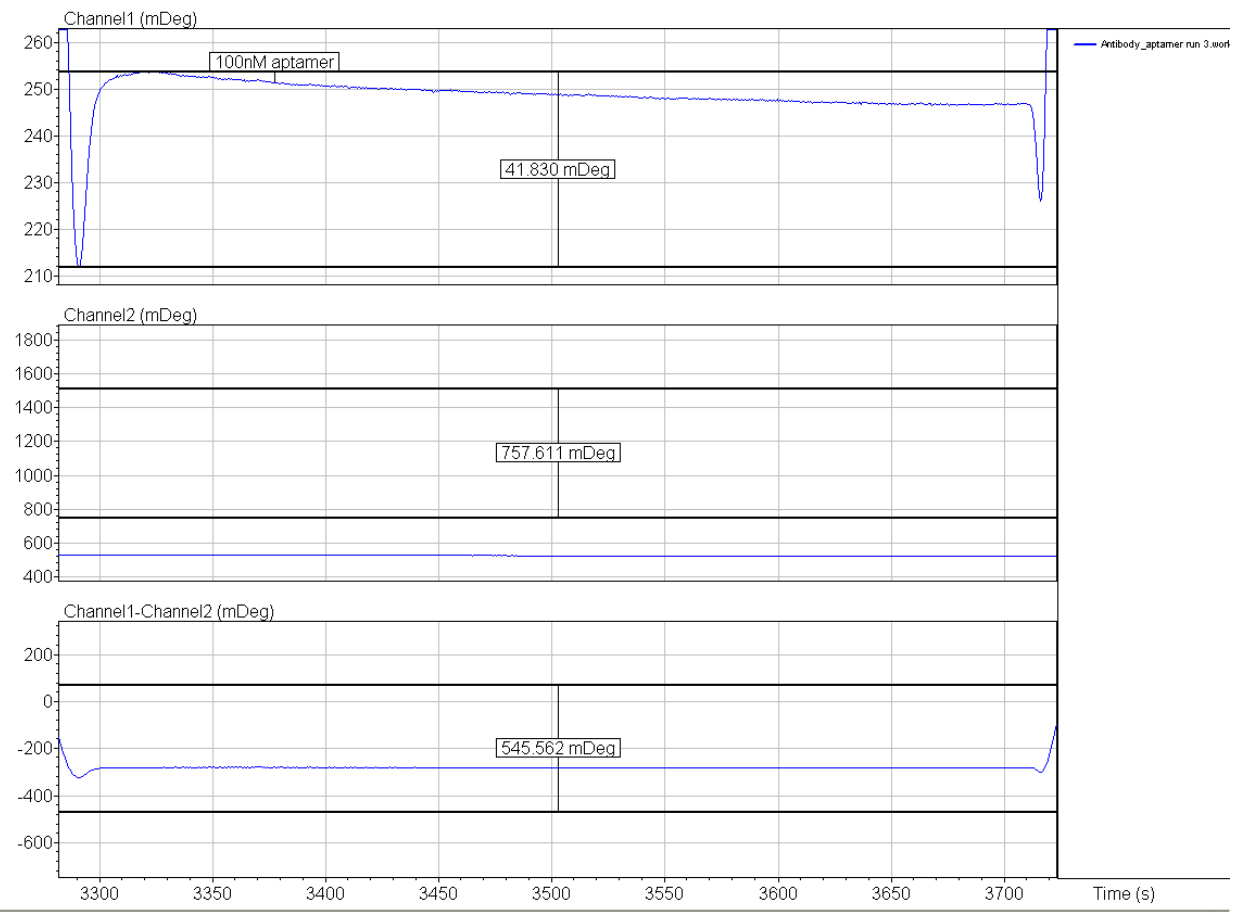

100nM aptamer qualitative analysis
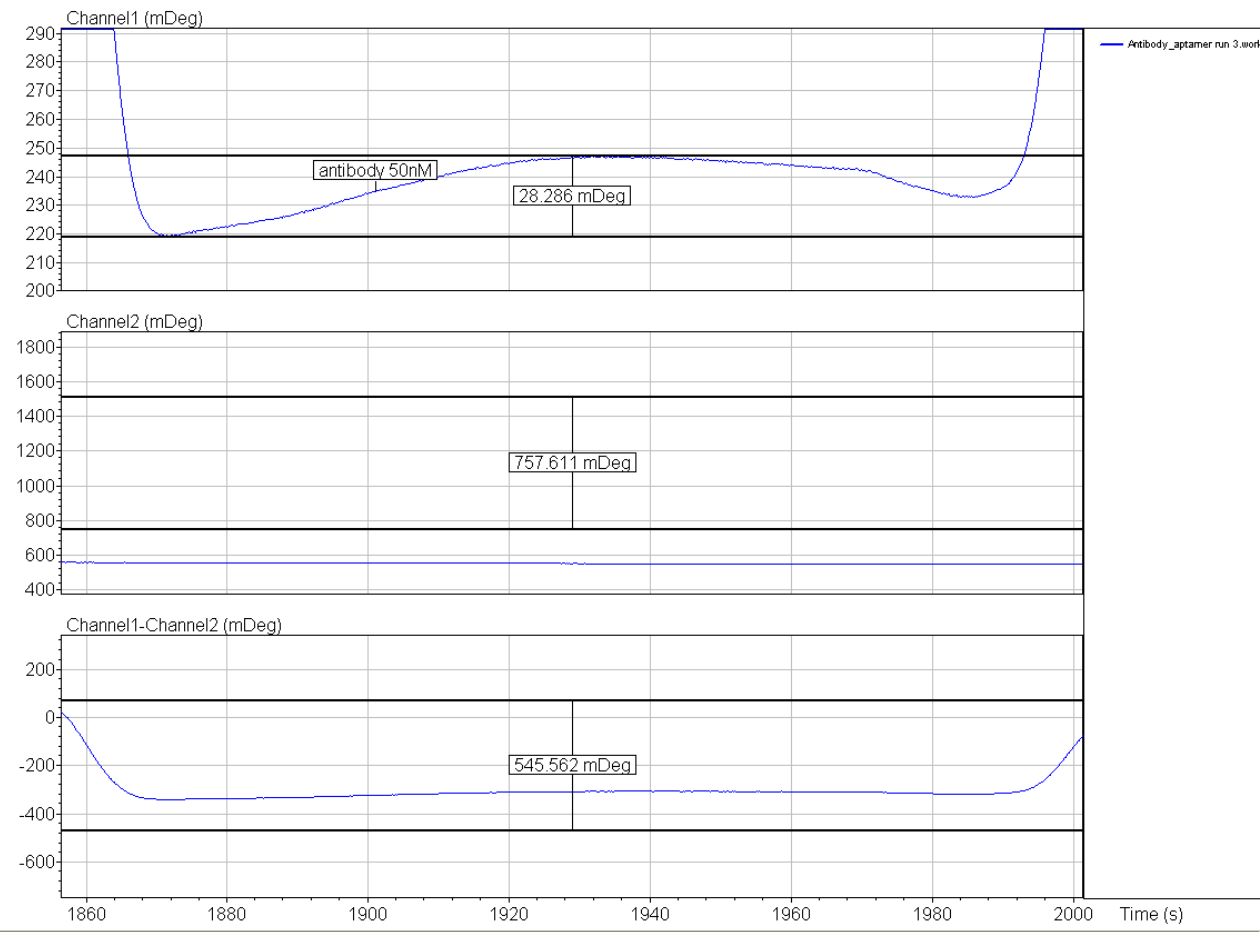

50nM antibody qualitative analysis 

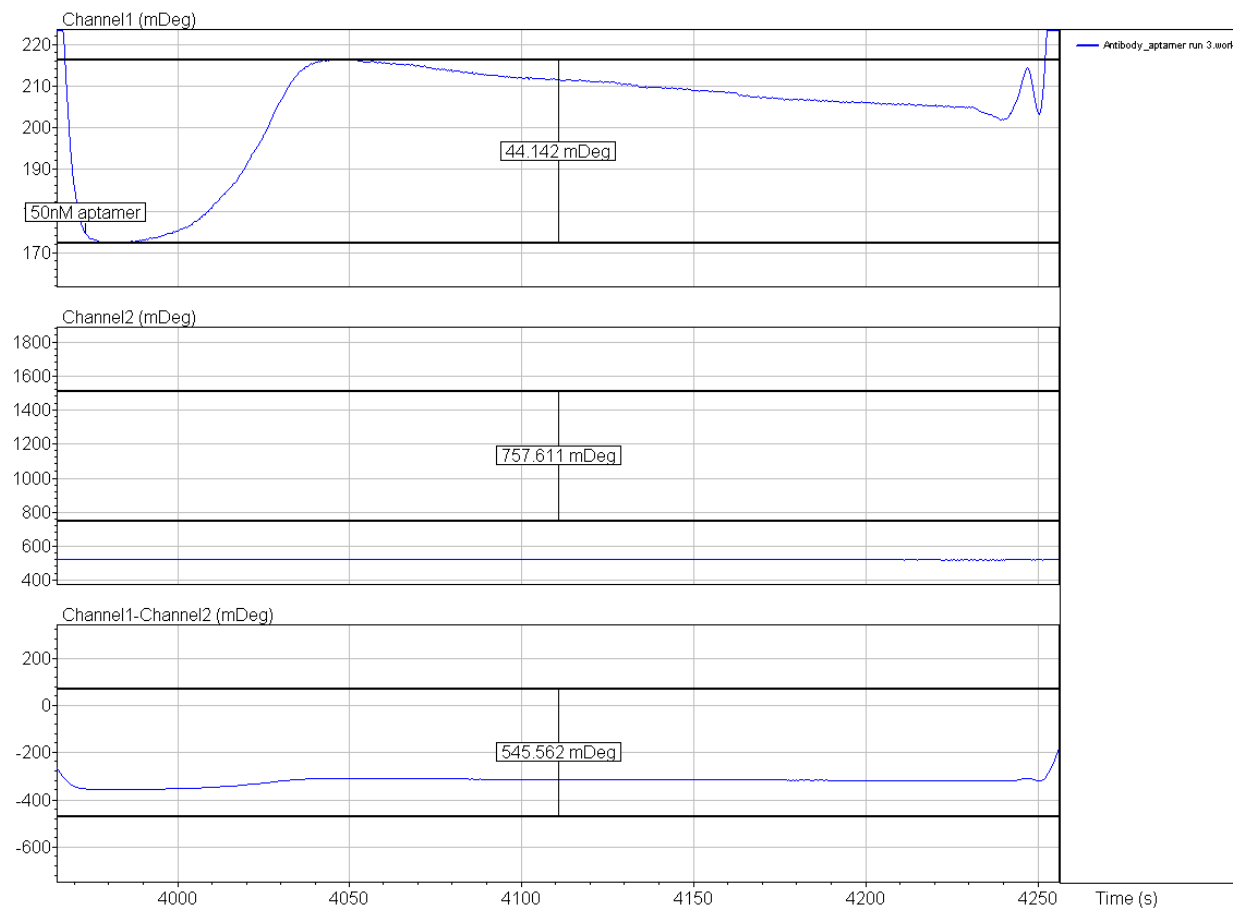

50nM aptamer qualitative analysis
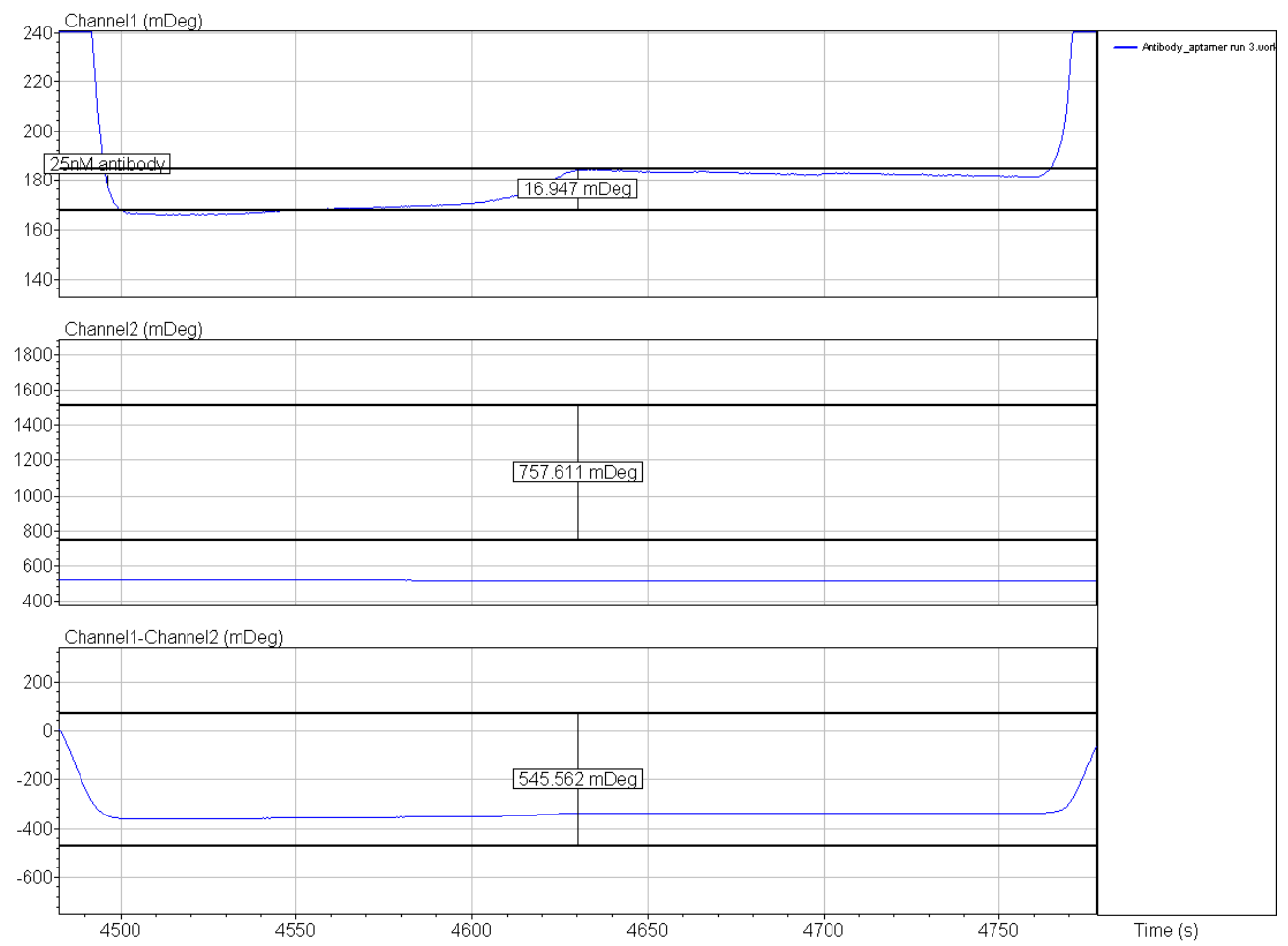

25nM antibody qualitative analysis 


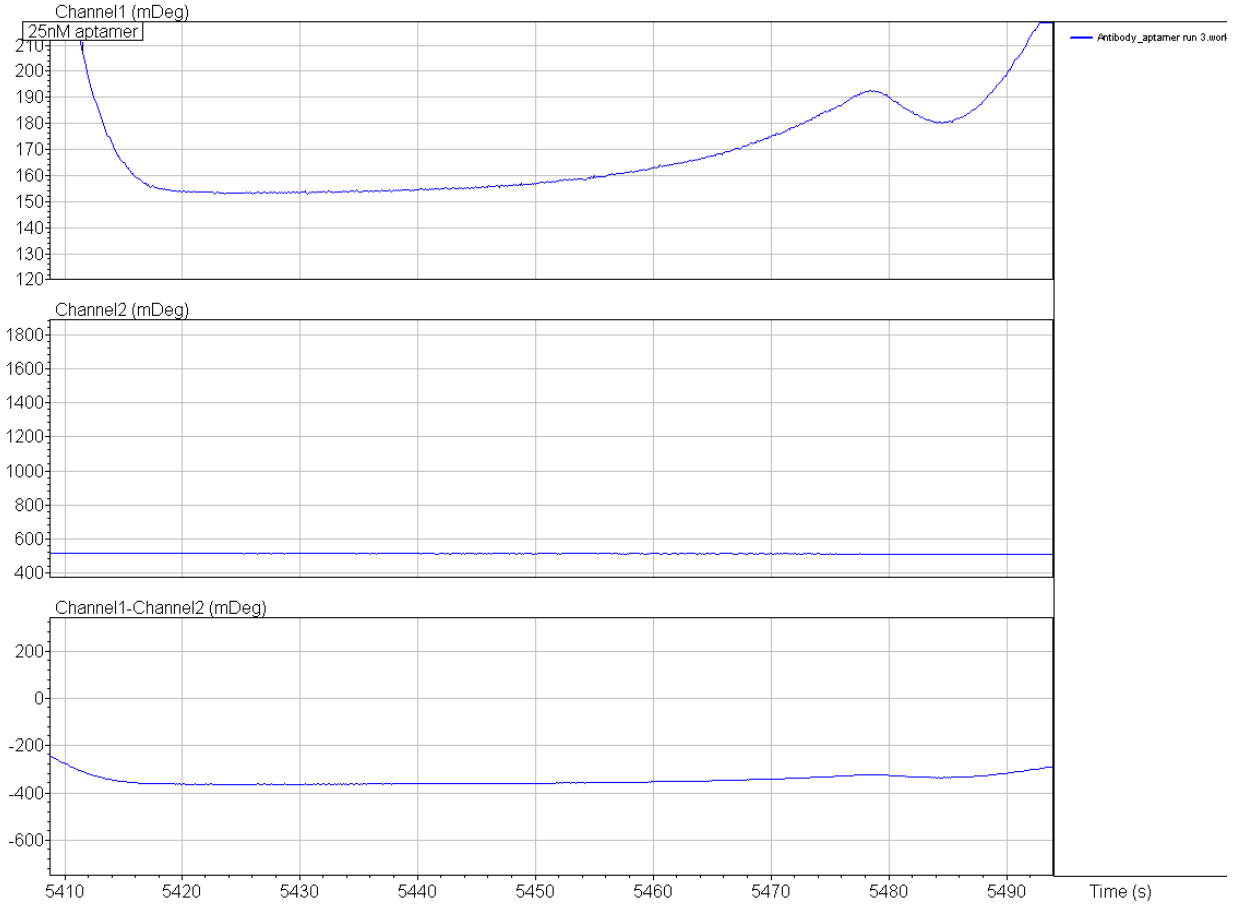

25nM aptamer qualitative analysis 


\section{F. SPR RUN 4 RAW DATA}
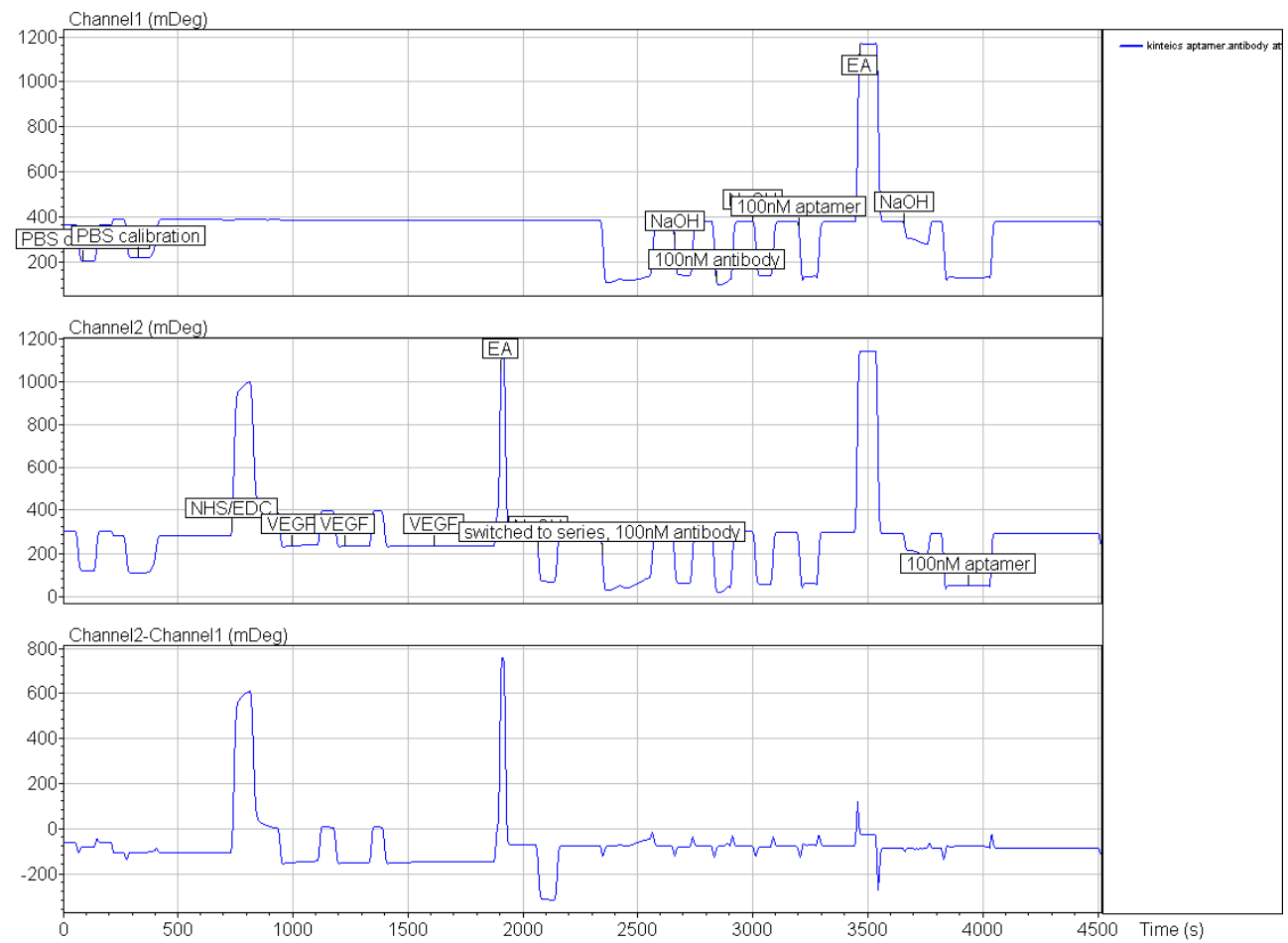

Overall SPR Run 4
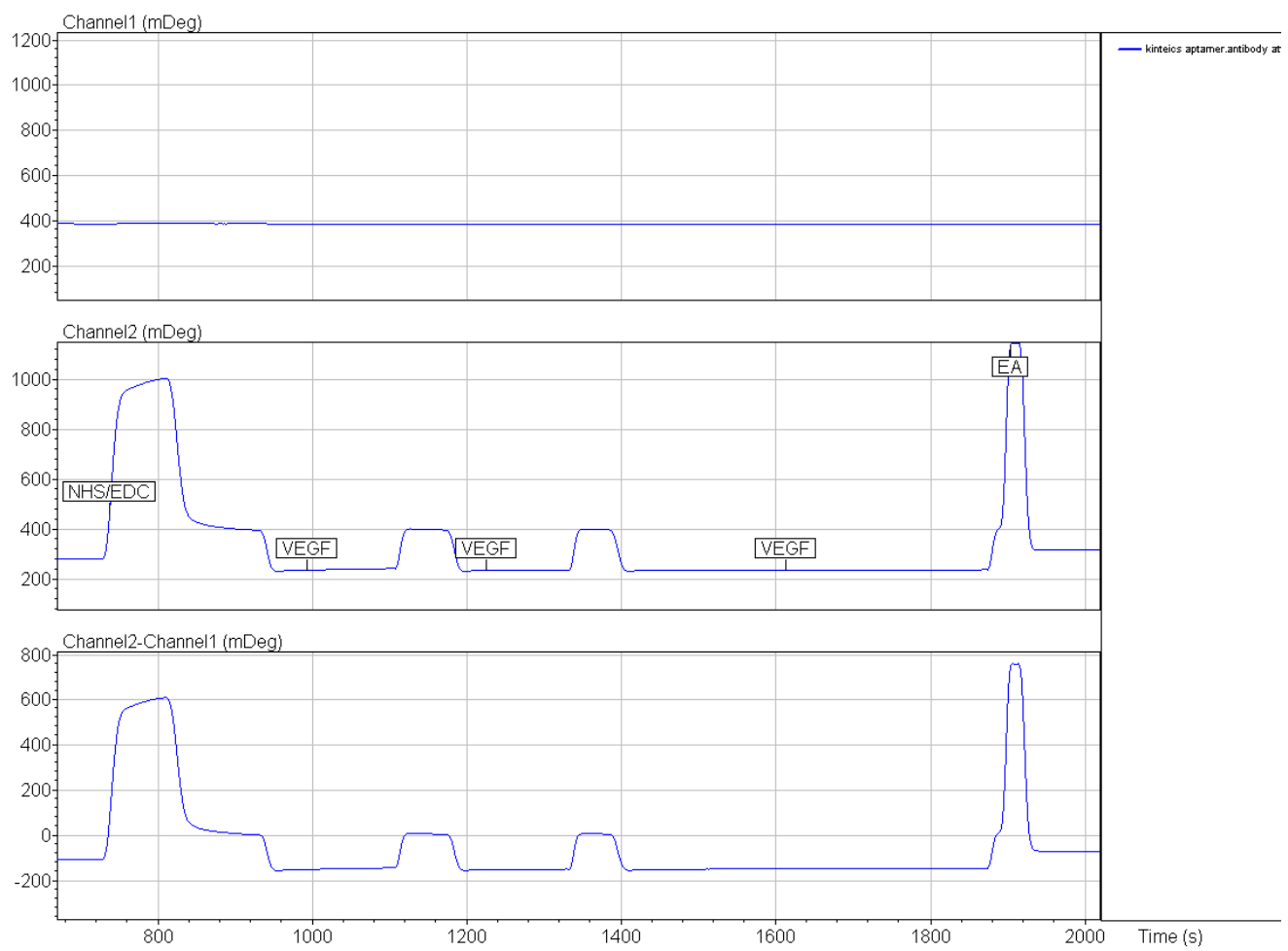

Activation of the CM-Dextran and immobilization of VEGF-A 

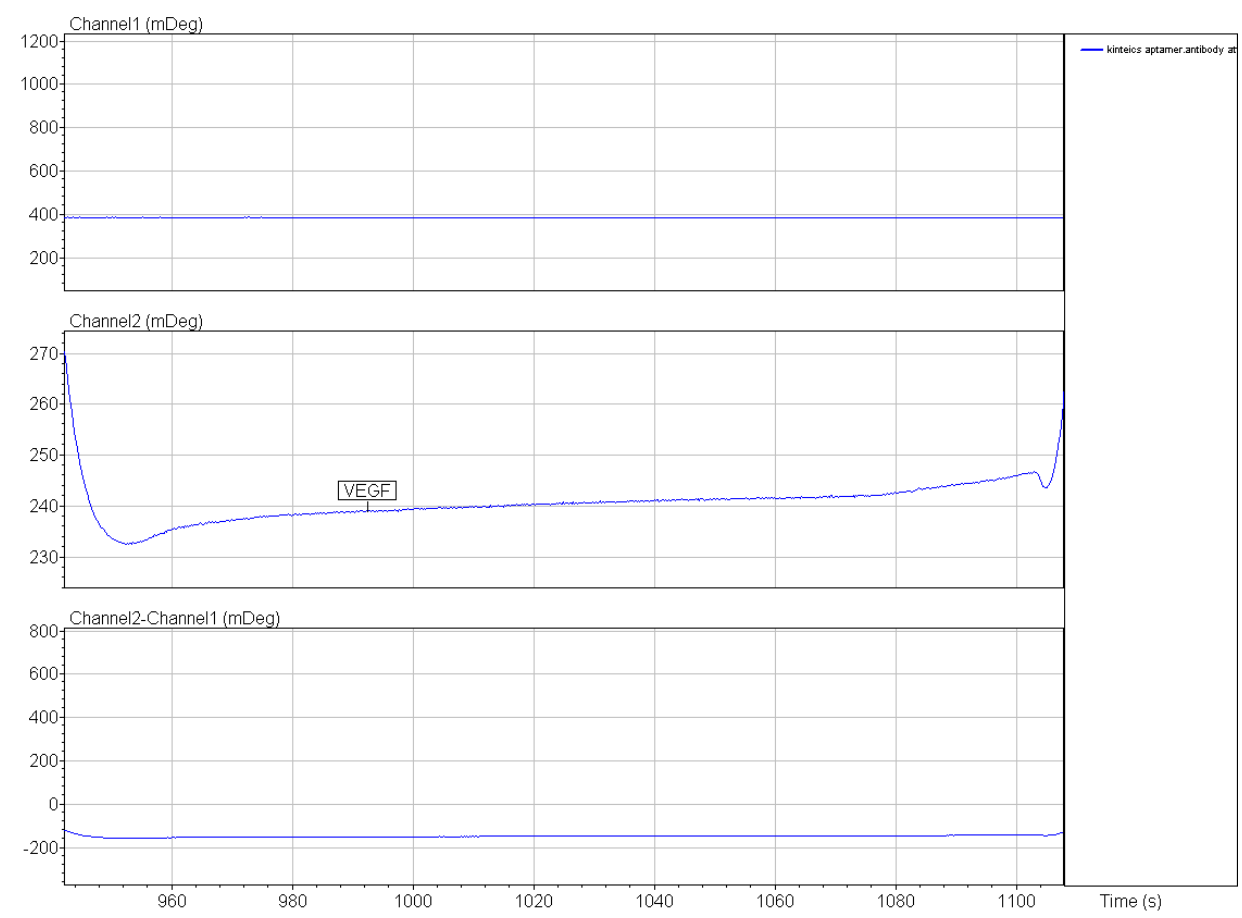

VEGF-A immobilization curve
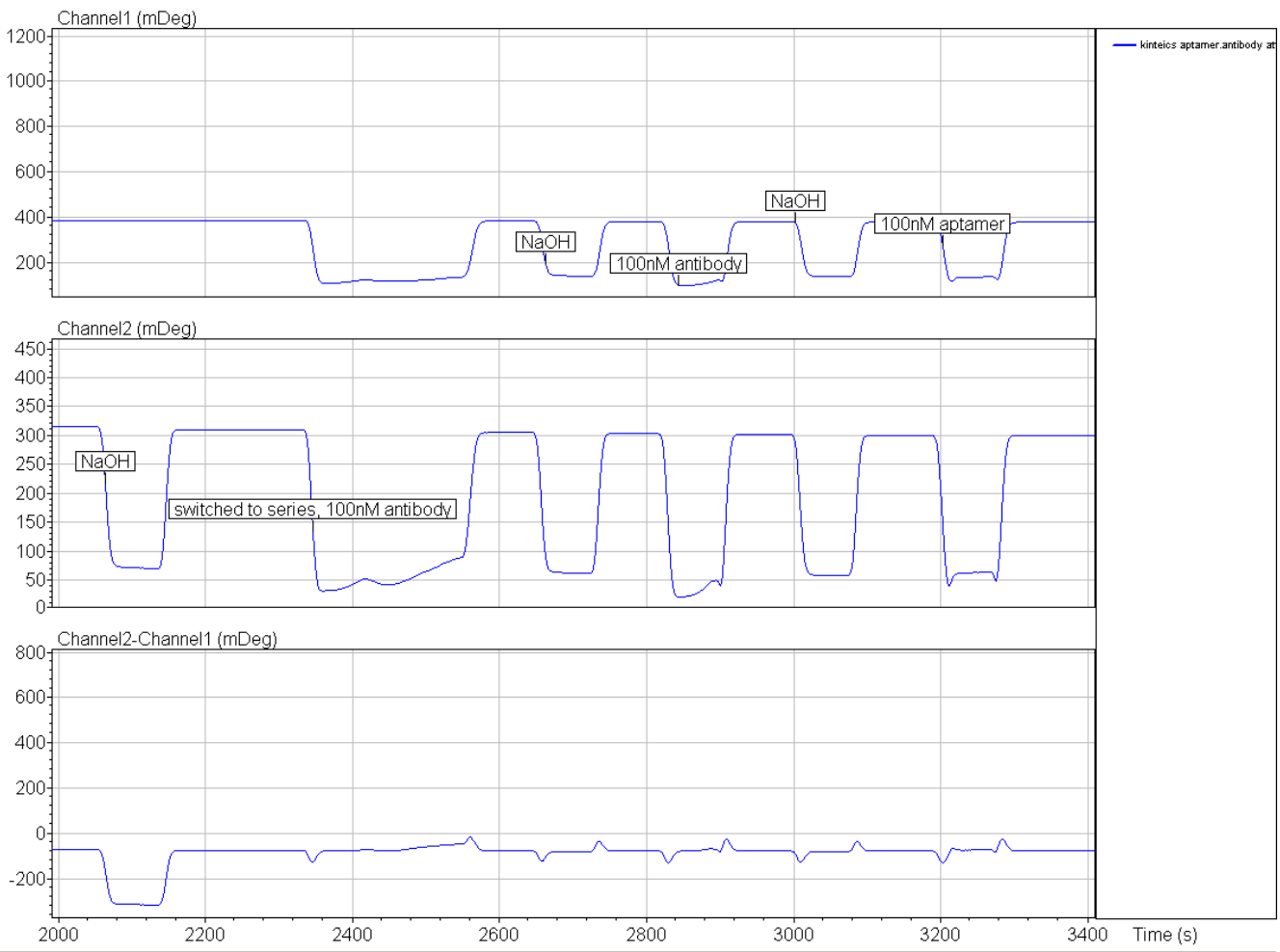

\section{Run 4 Injections}



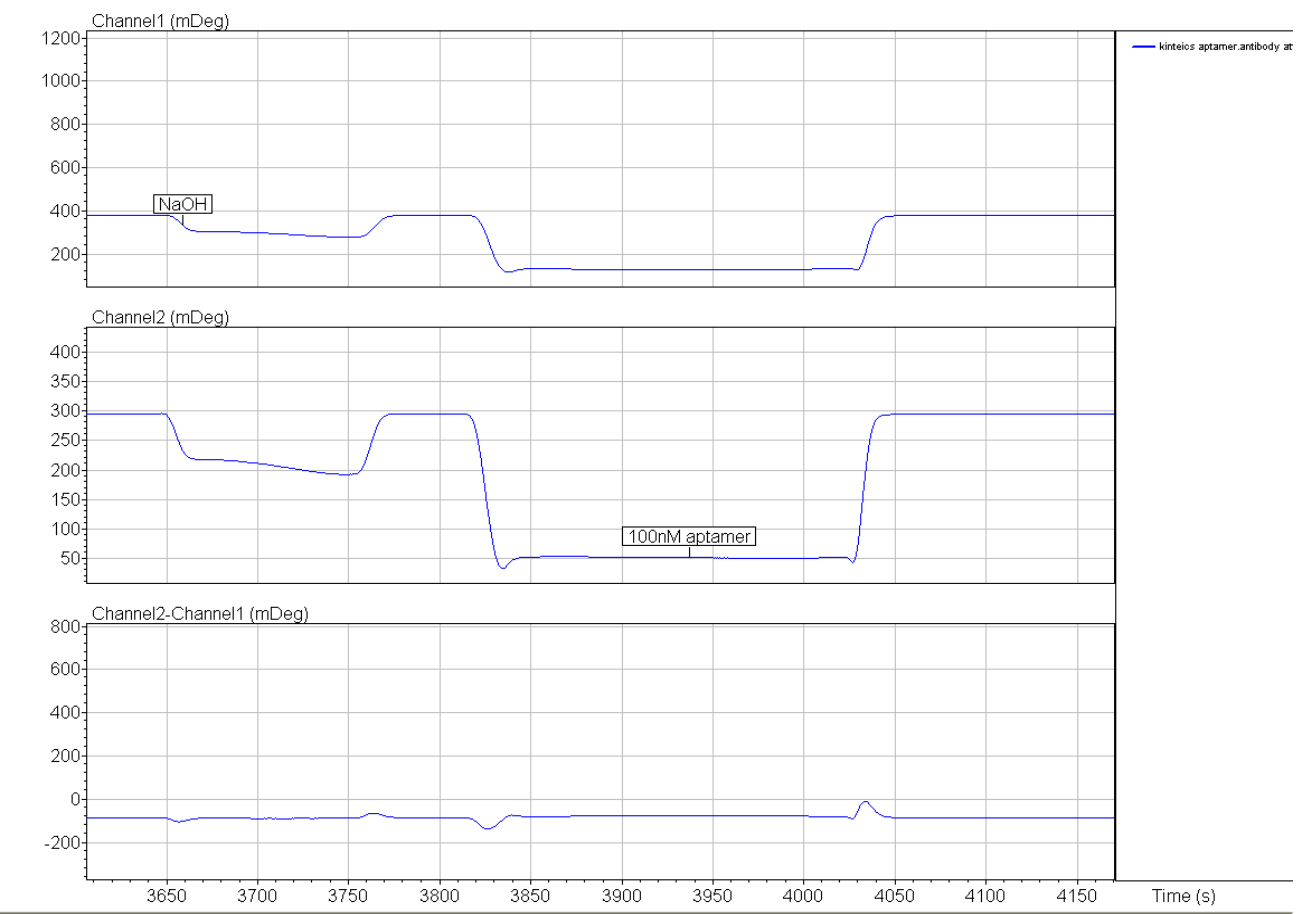

100nM binding curve

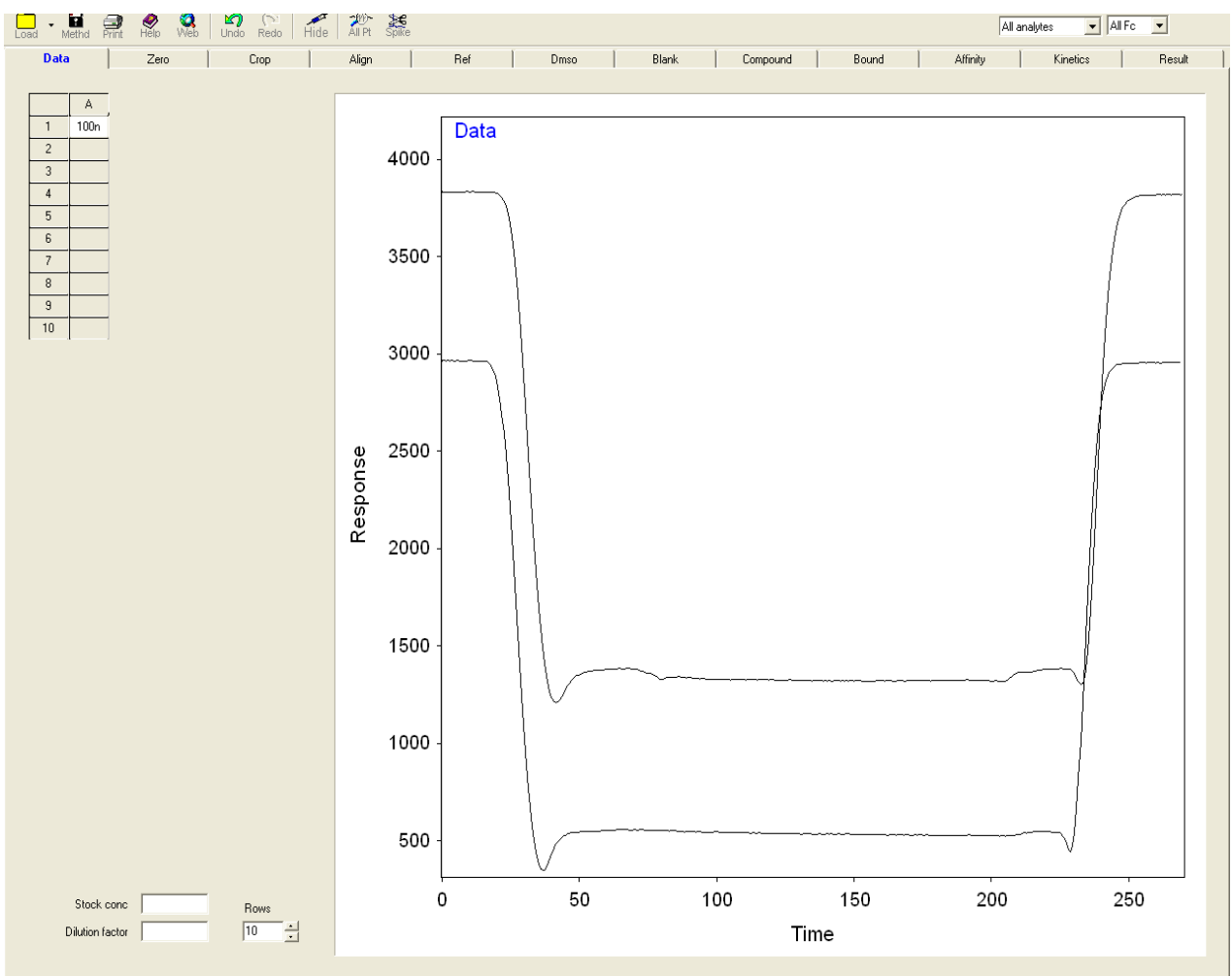

100nM aptamer kinetic analysis page 1 


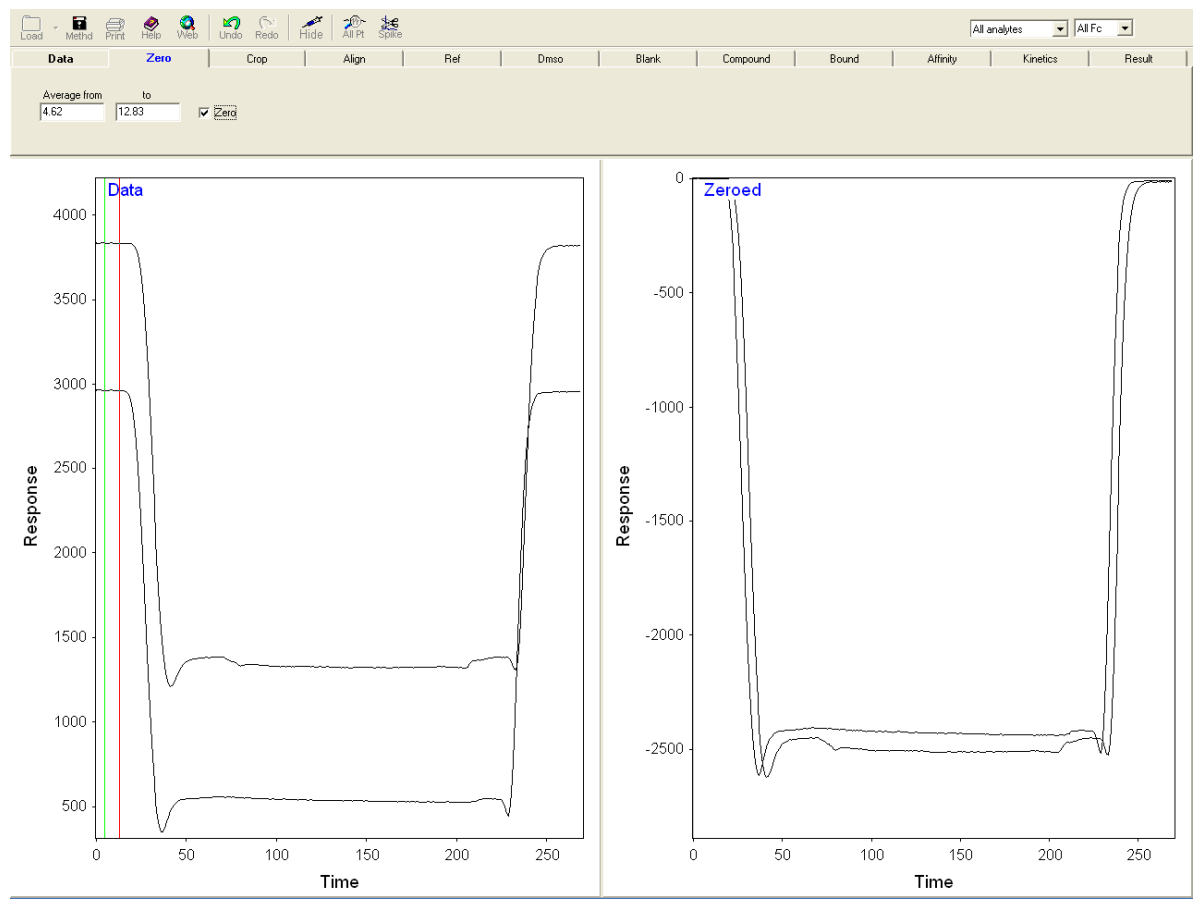

$100 \mathrm{nM}$ aptamer kinetic analysis page 2
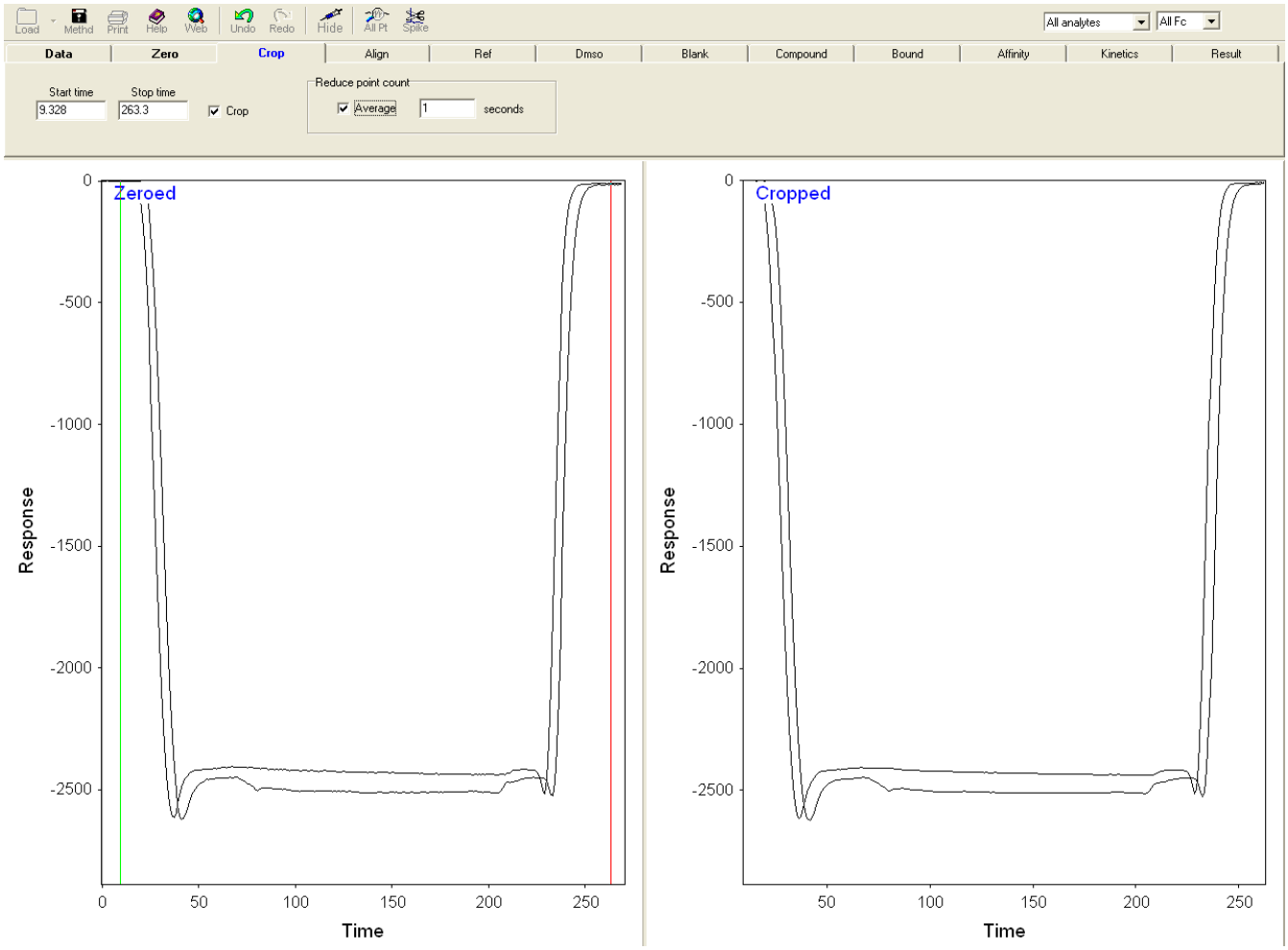

100nM antamer kinetic analvsis nage 3 


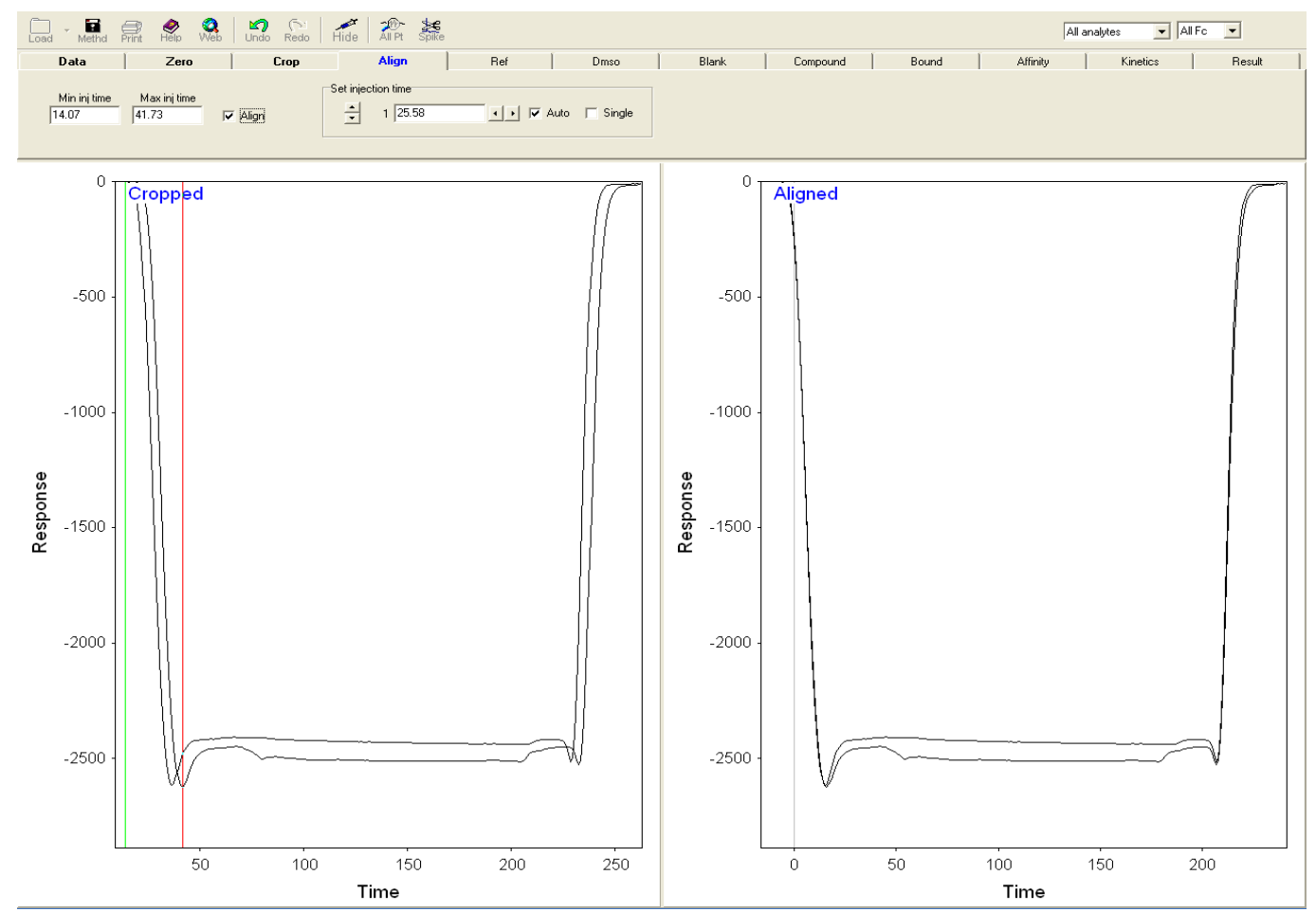

100nM abtamer kinetic analvsis bage 4

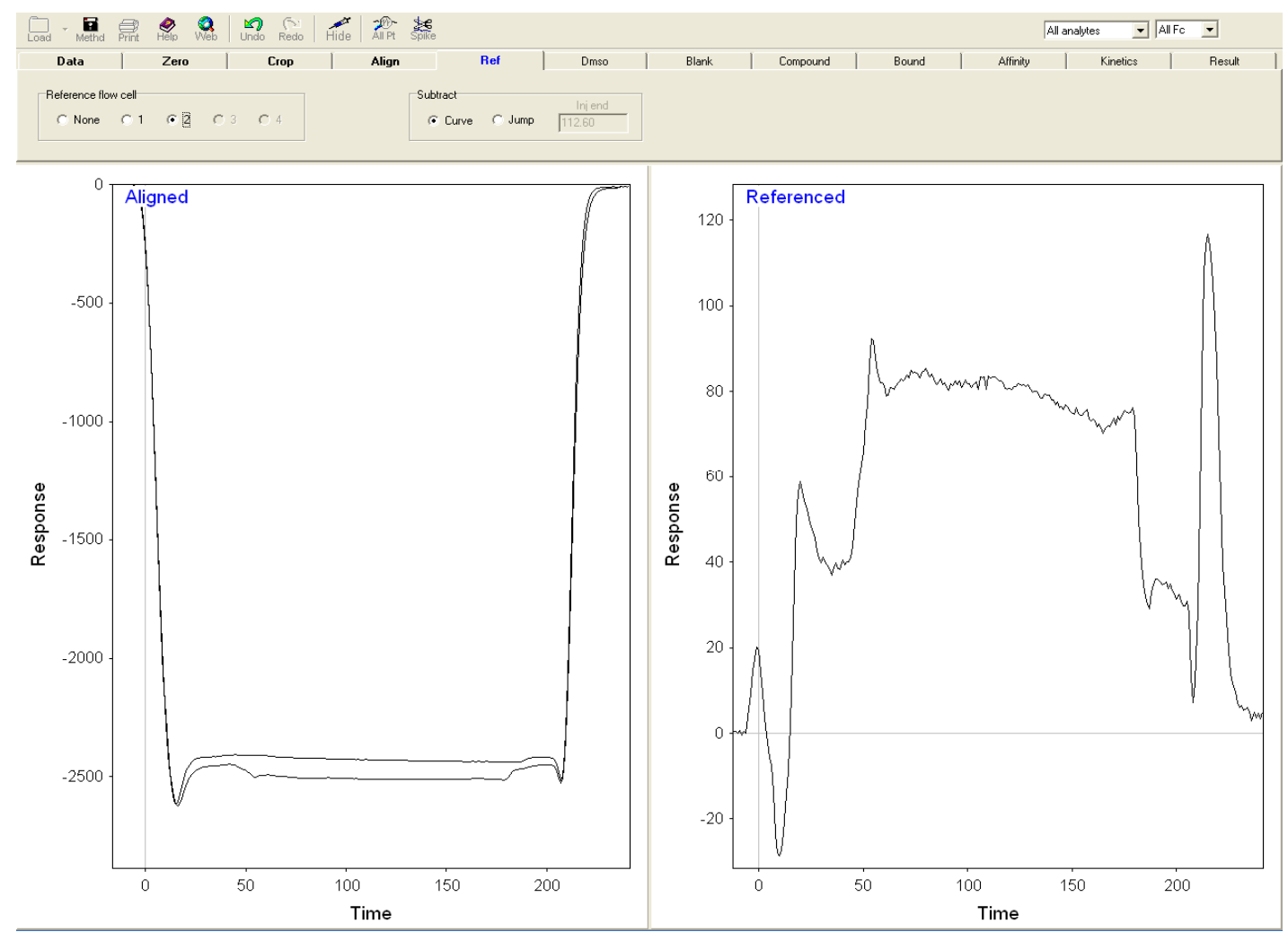

100nM aptamer kinetic analvsis page 5 


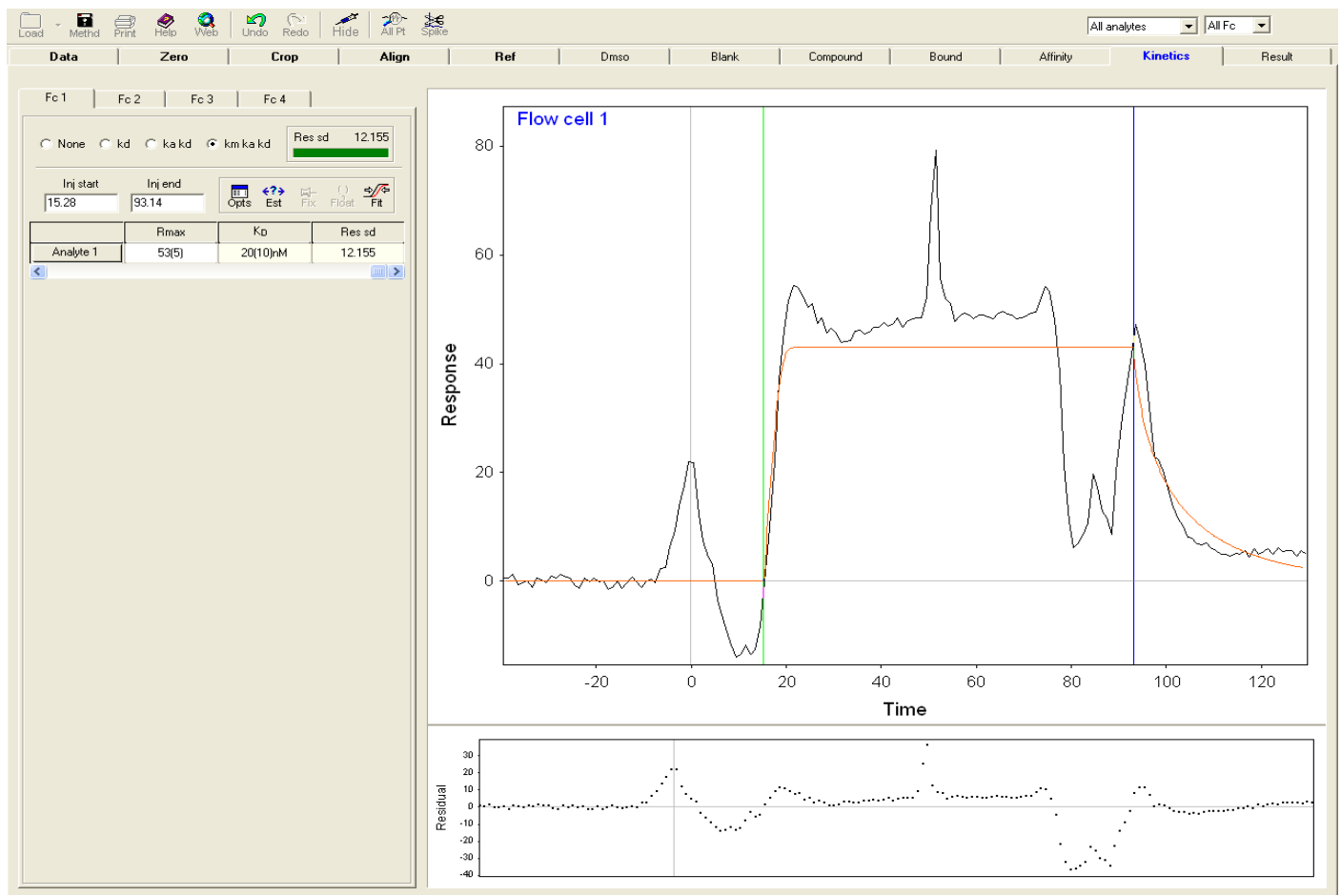

$100 \mathrm{nM}$ antamer kinetic analvsis nage 6

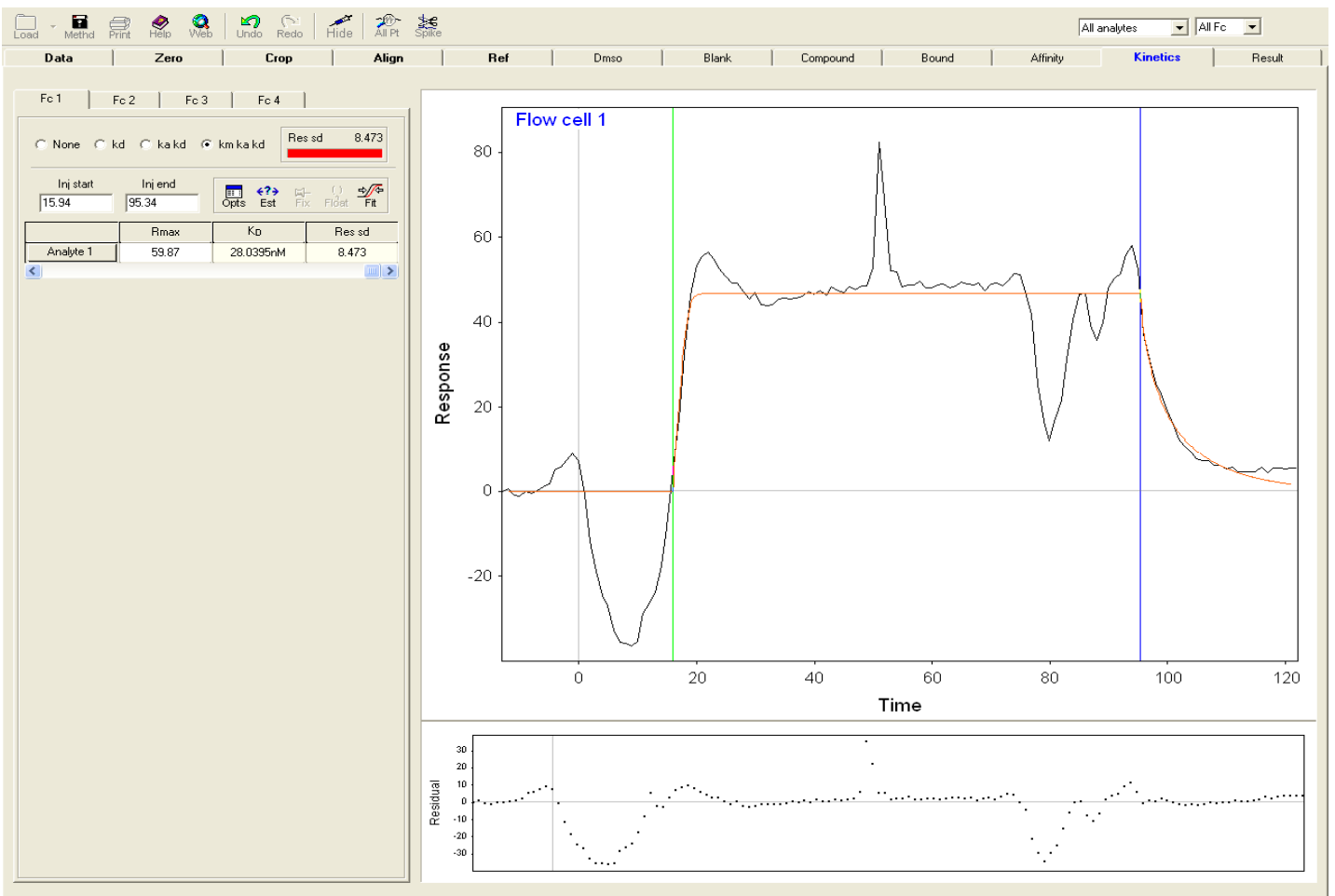

100nM aptamer kinetic analysis 2 


\section{G. SPR Run 5 RAW DATA}

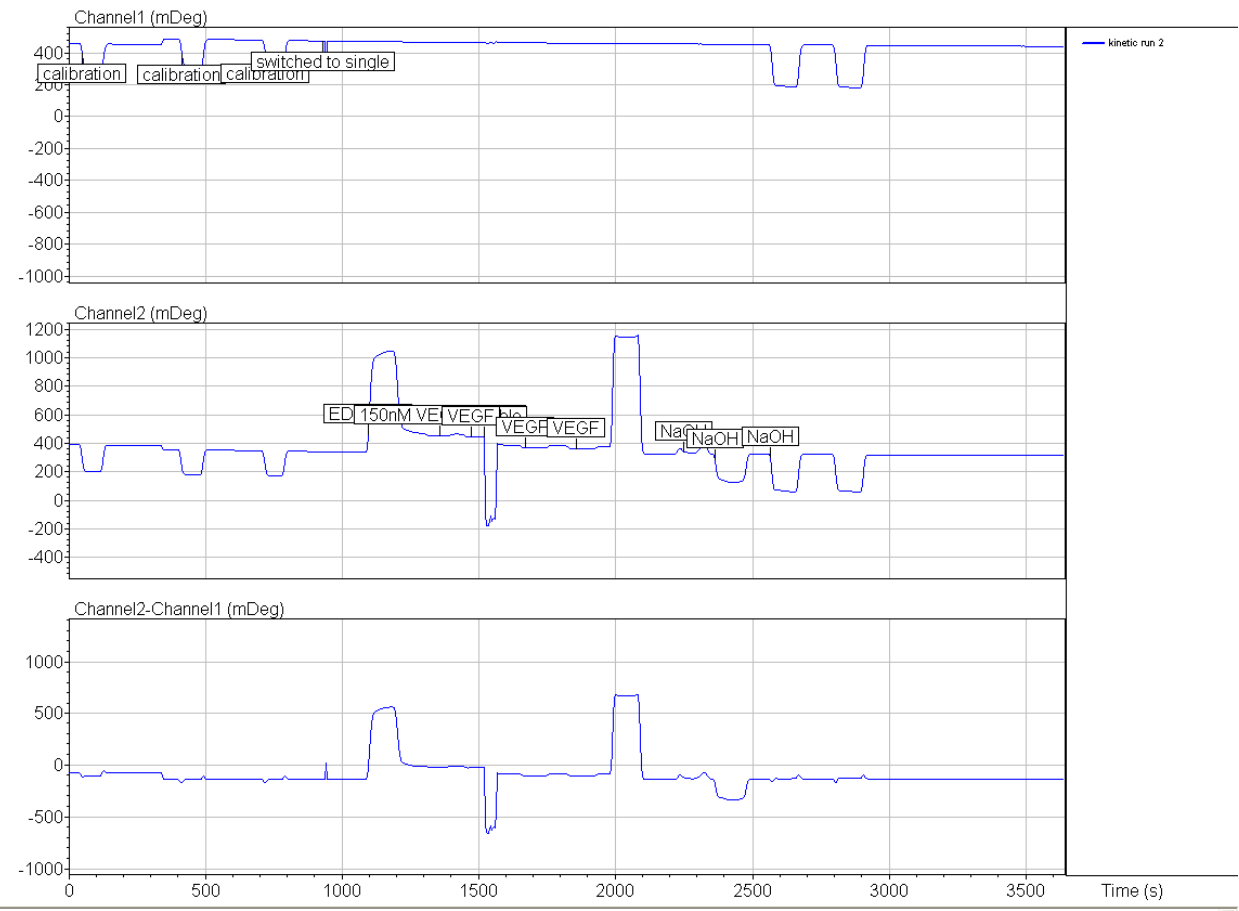

Overall SPR Run 5
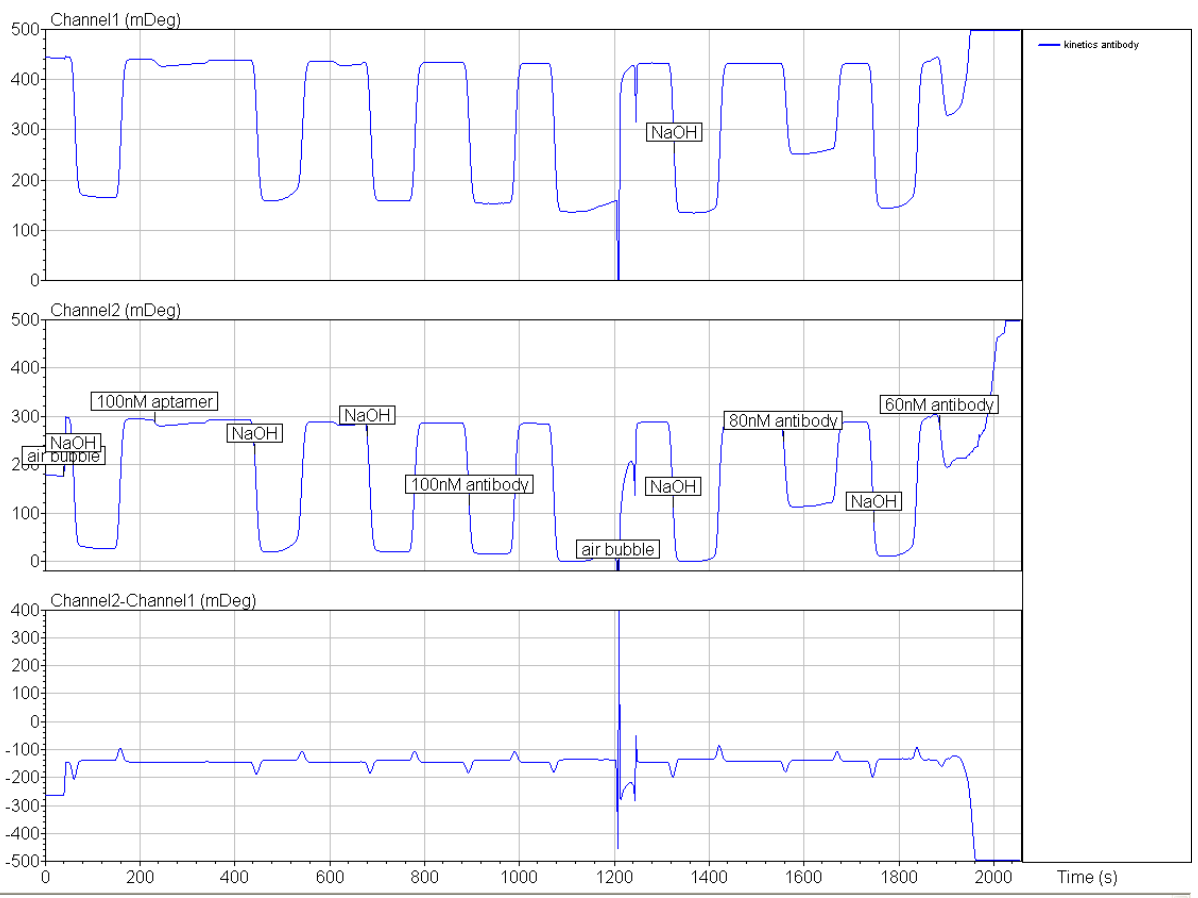

Run 5 injections page 1 


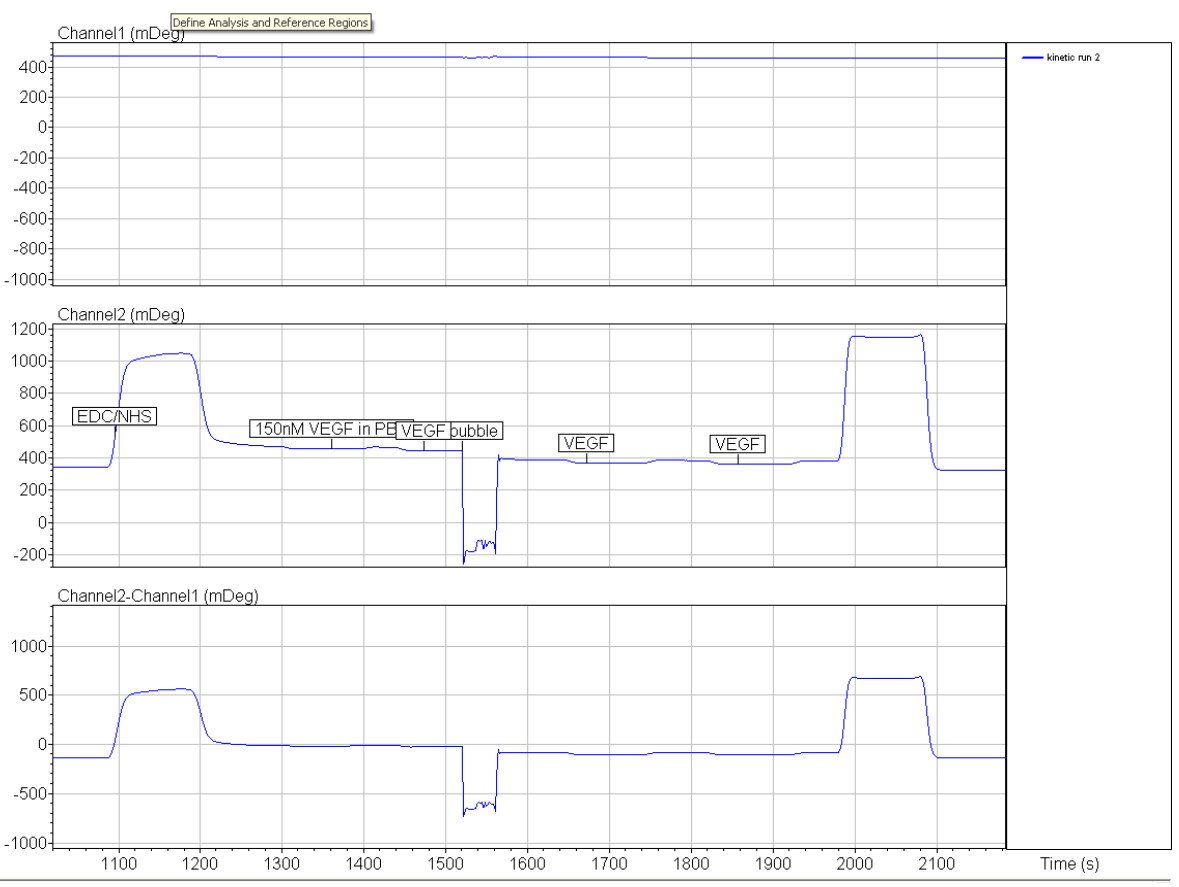

Run 5 injections page 2

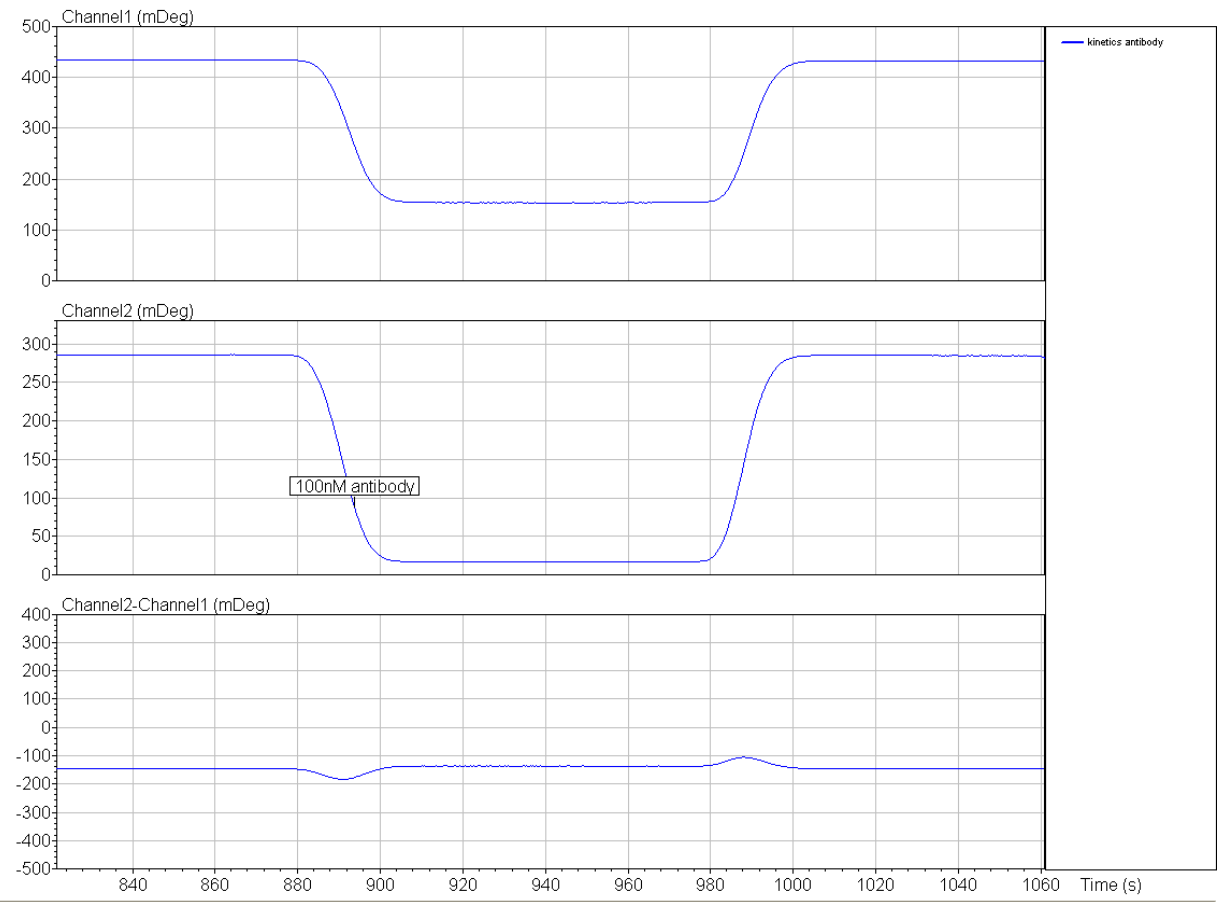

100nM antibody binding curve 


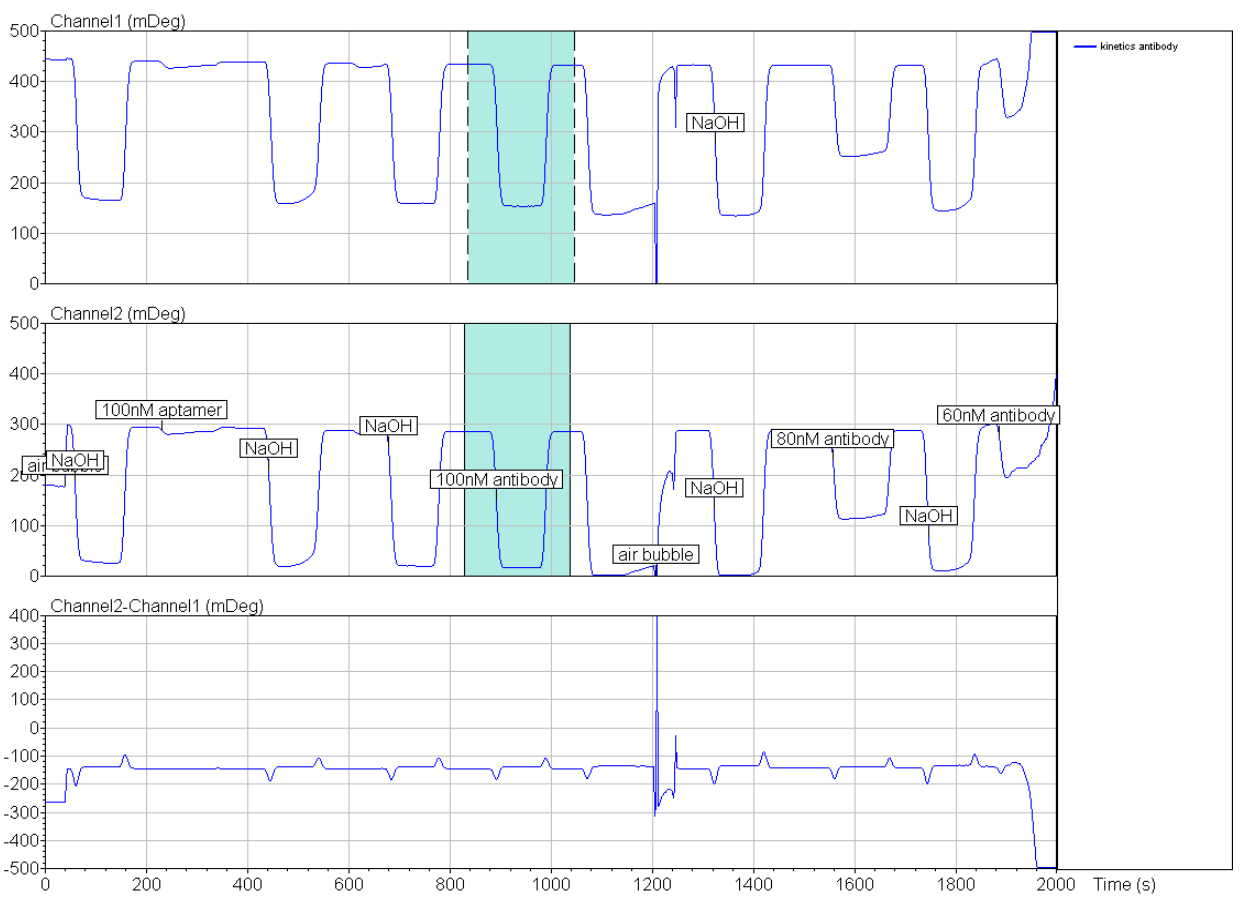

Selection of the curve to be analyzed and the reference to be

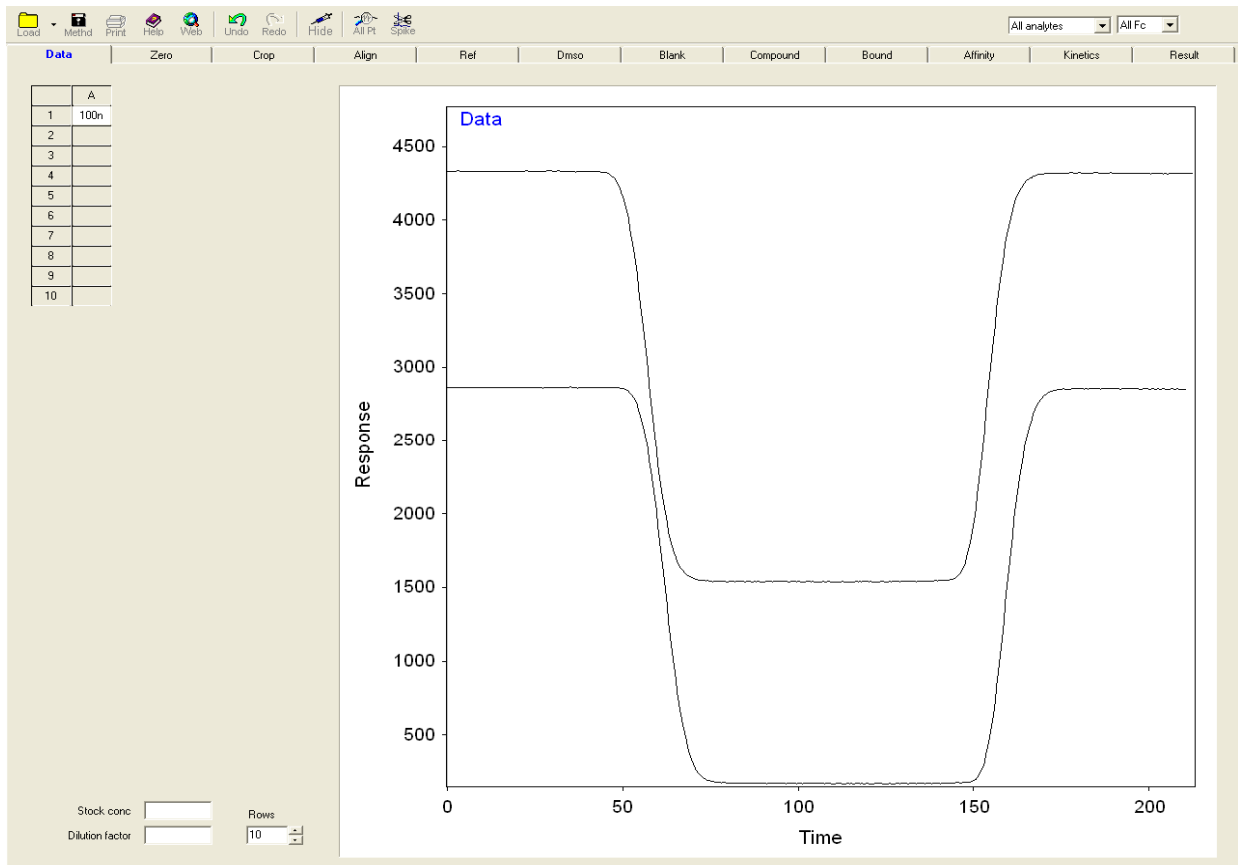

100nM antibody kinetic analysis page 1 

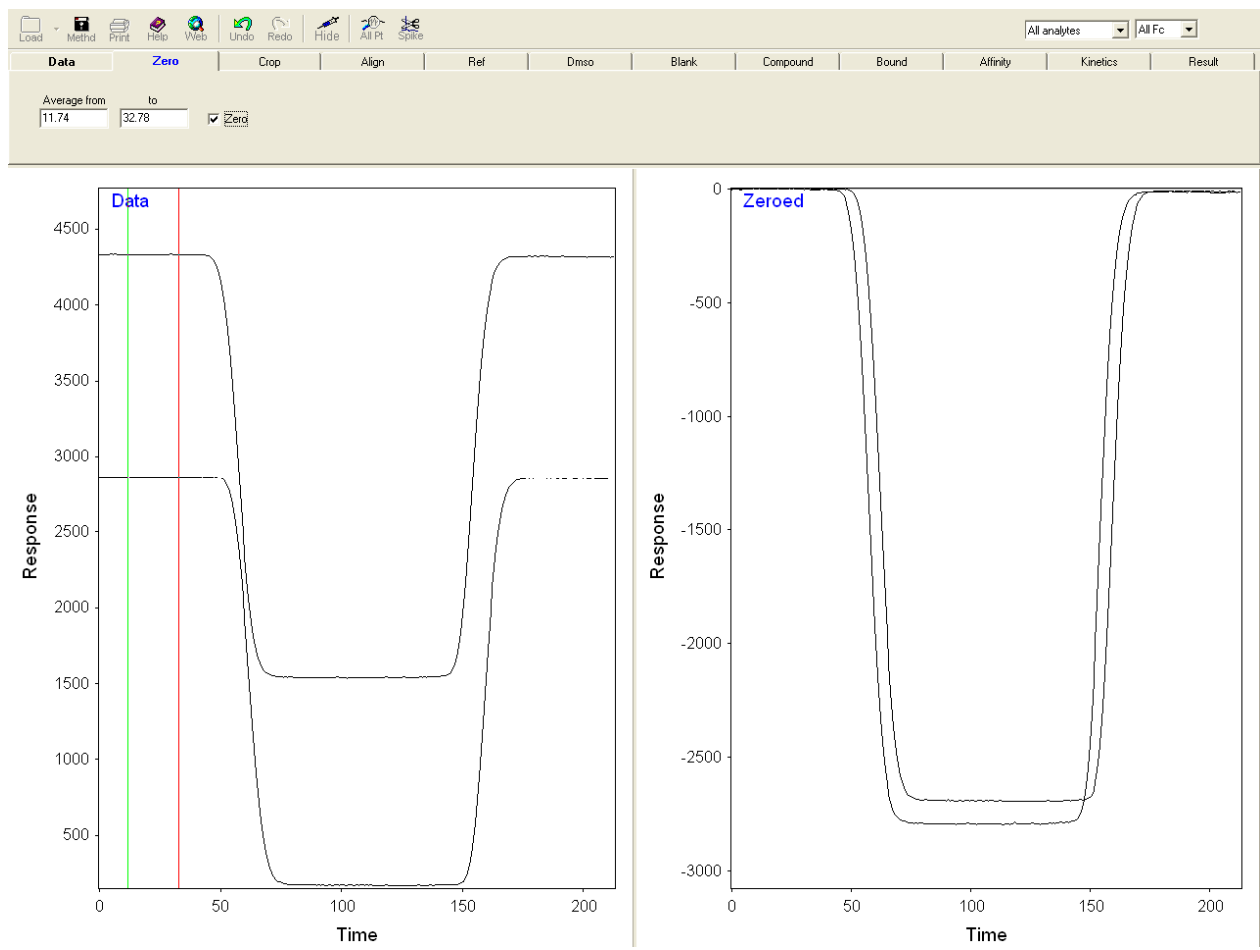

100nM antibody kinetic analysis page 2
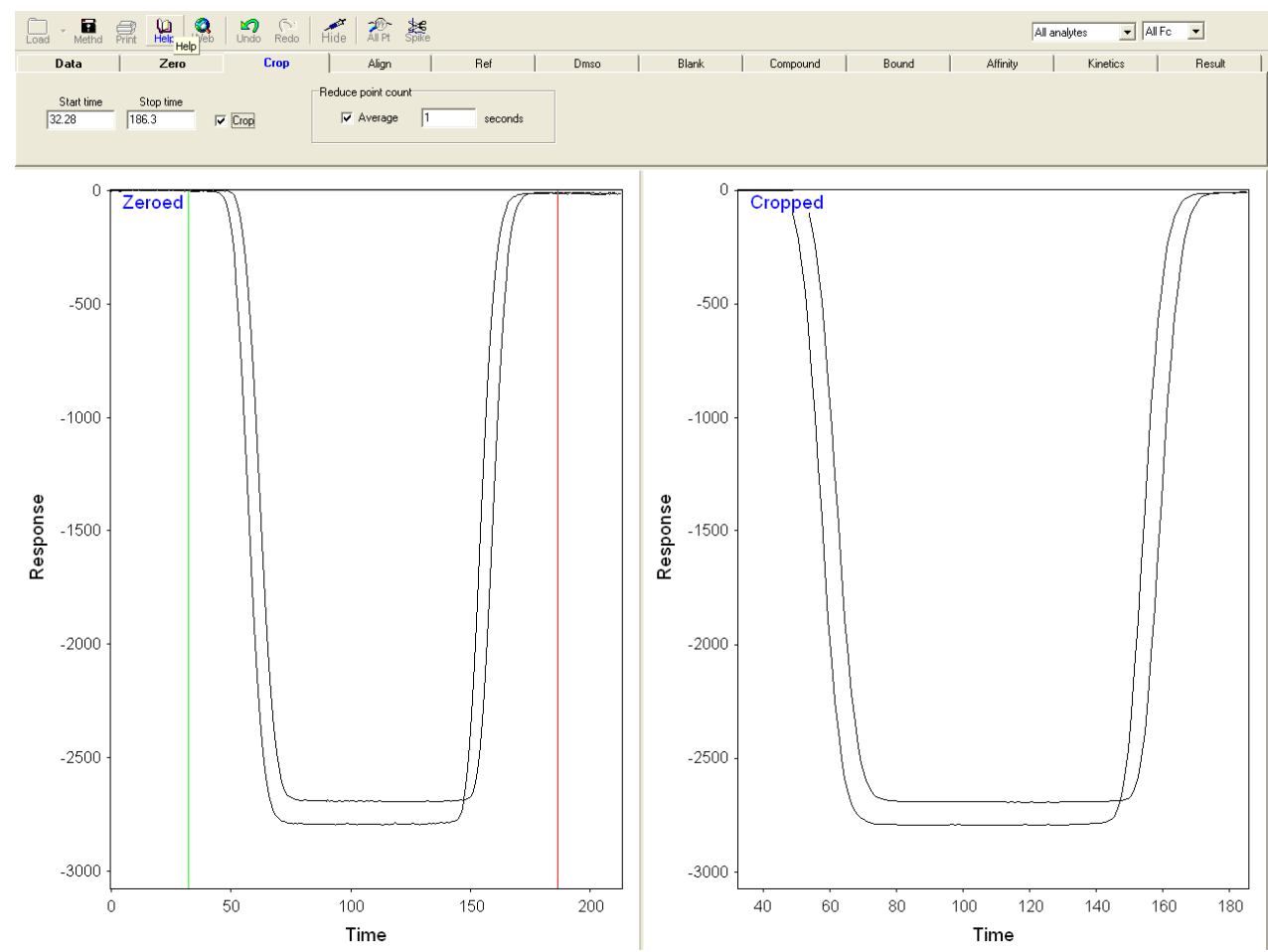

100nM antibody kinetic analysis page 3 


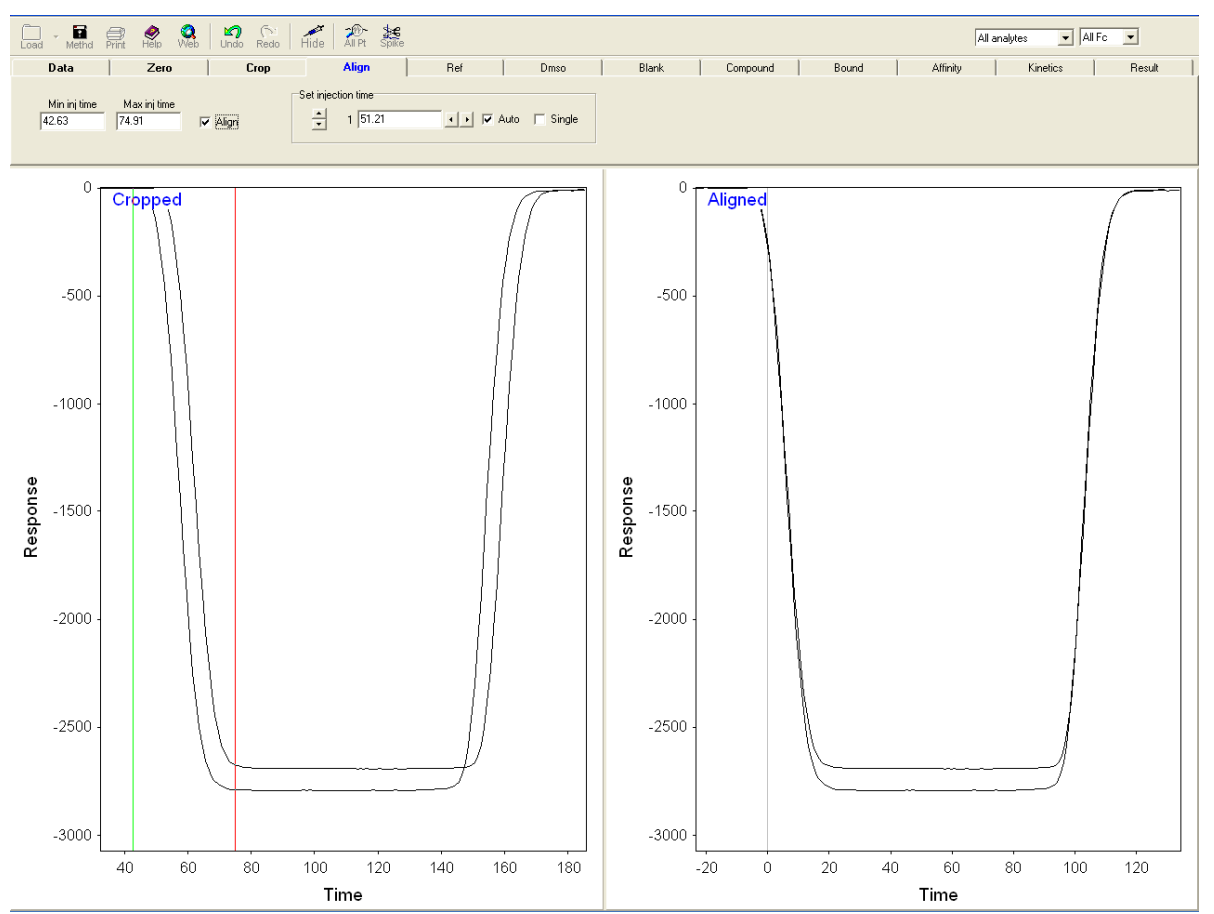

100nM antibody kinetic analysis page 4

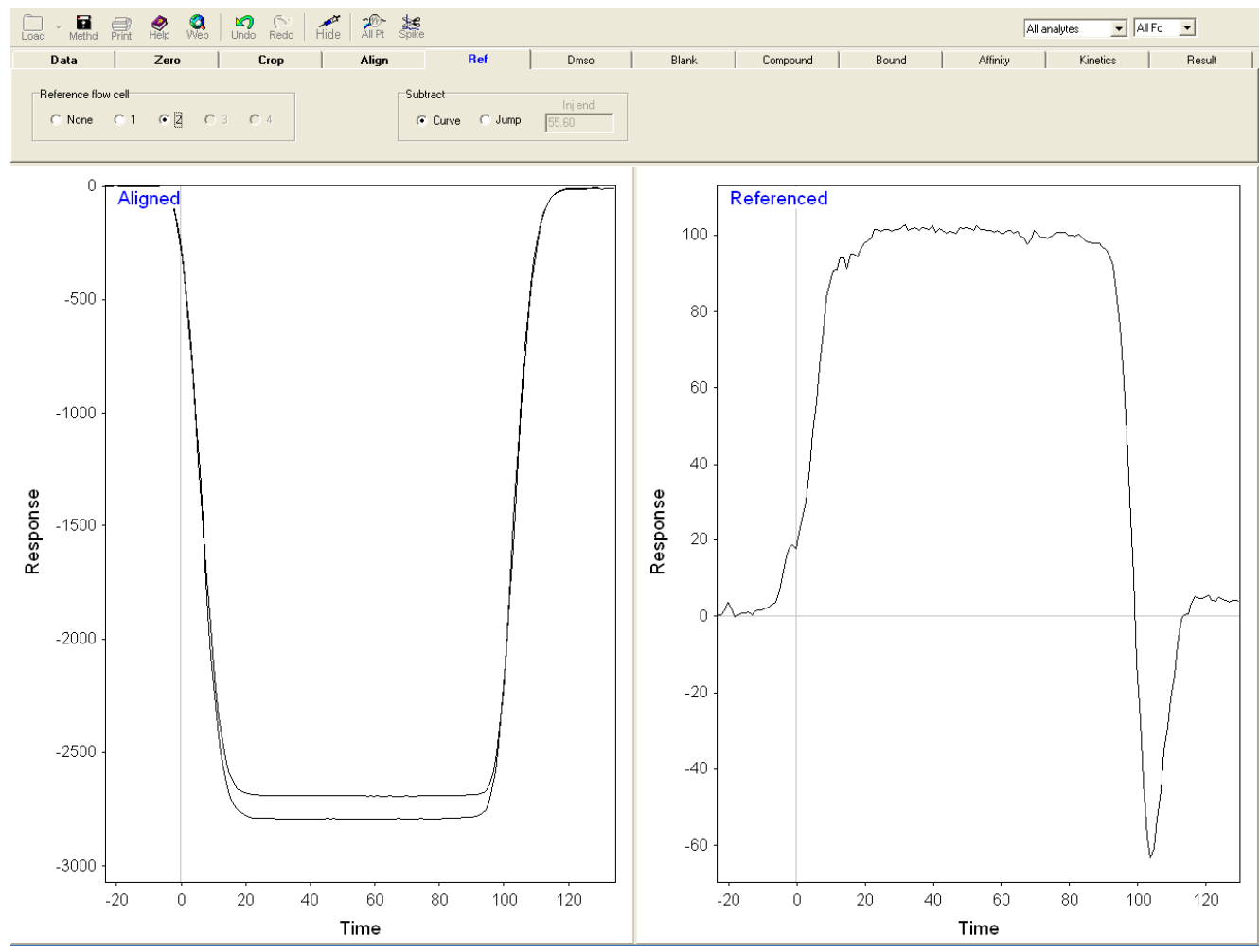

100nM antibody kinetic analysis page 5 


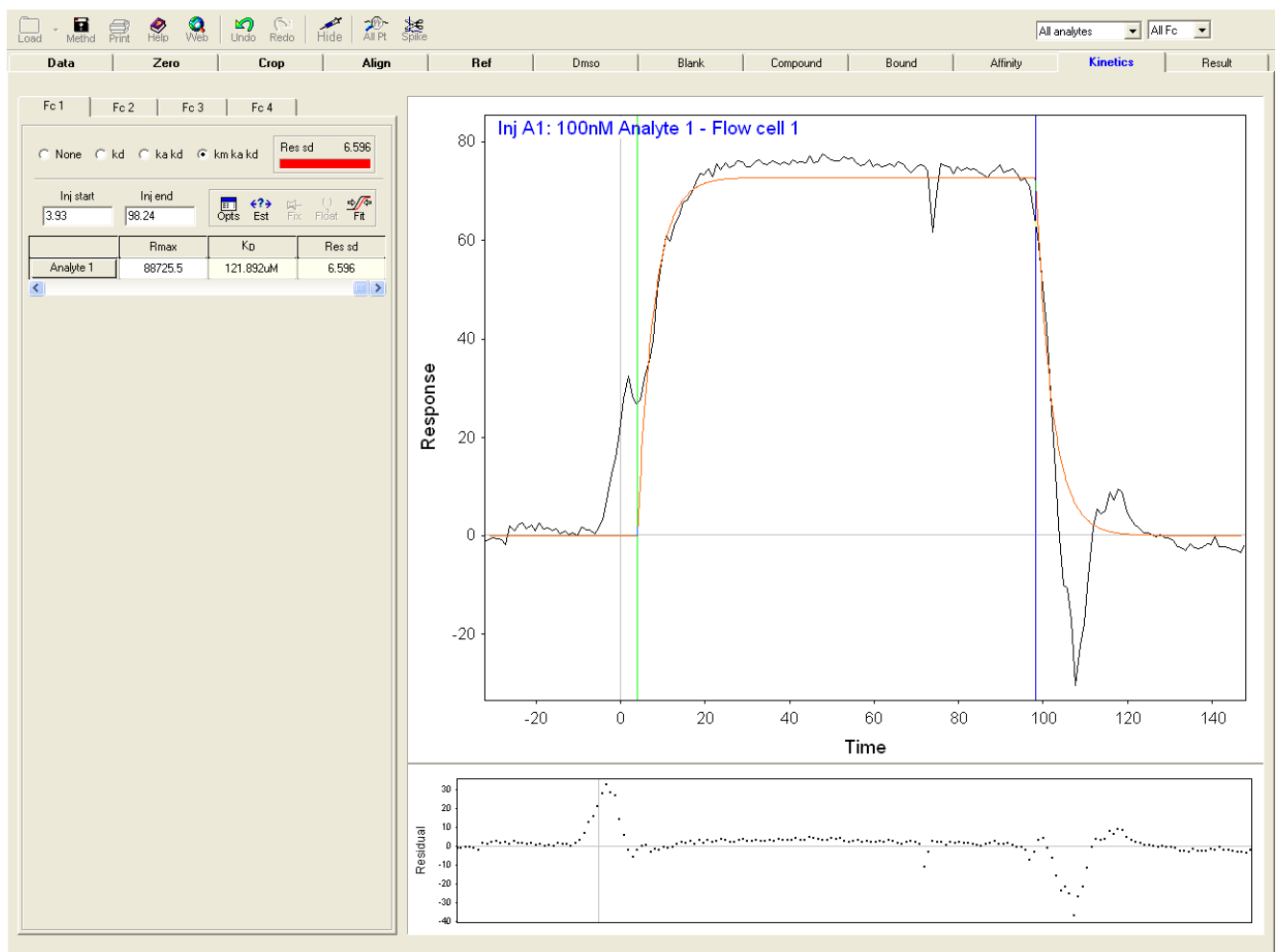

100nM antibody kinetic analysis page 6

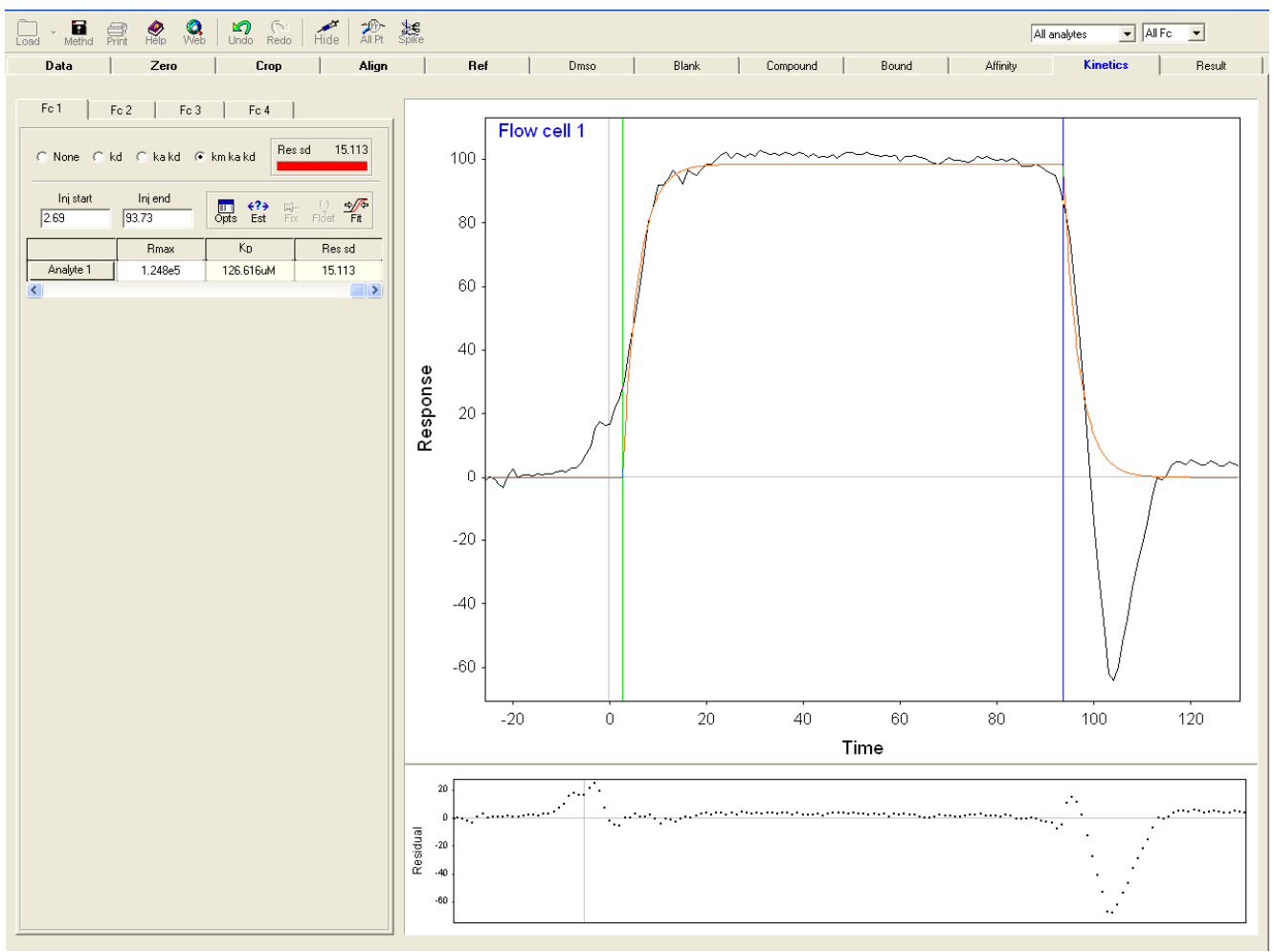

100nM antibody kinetic analysis page 7 


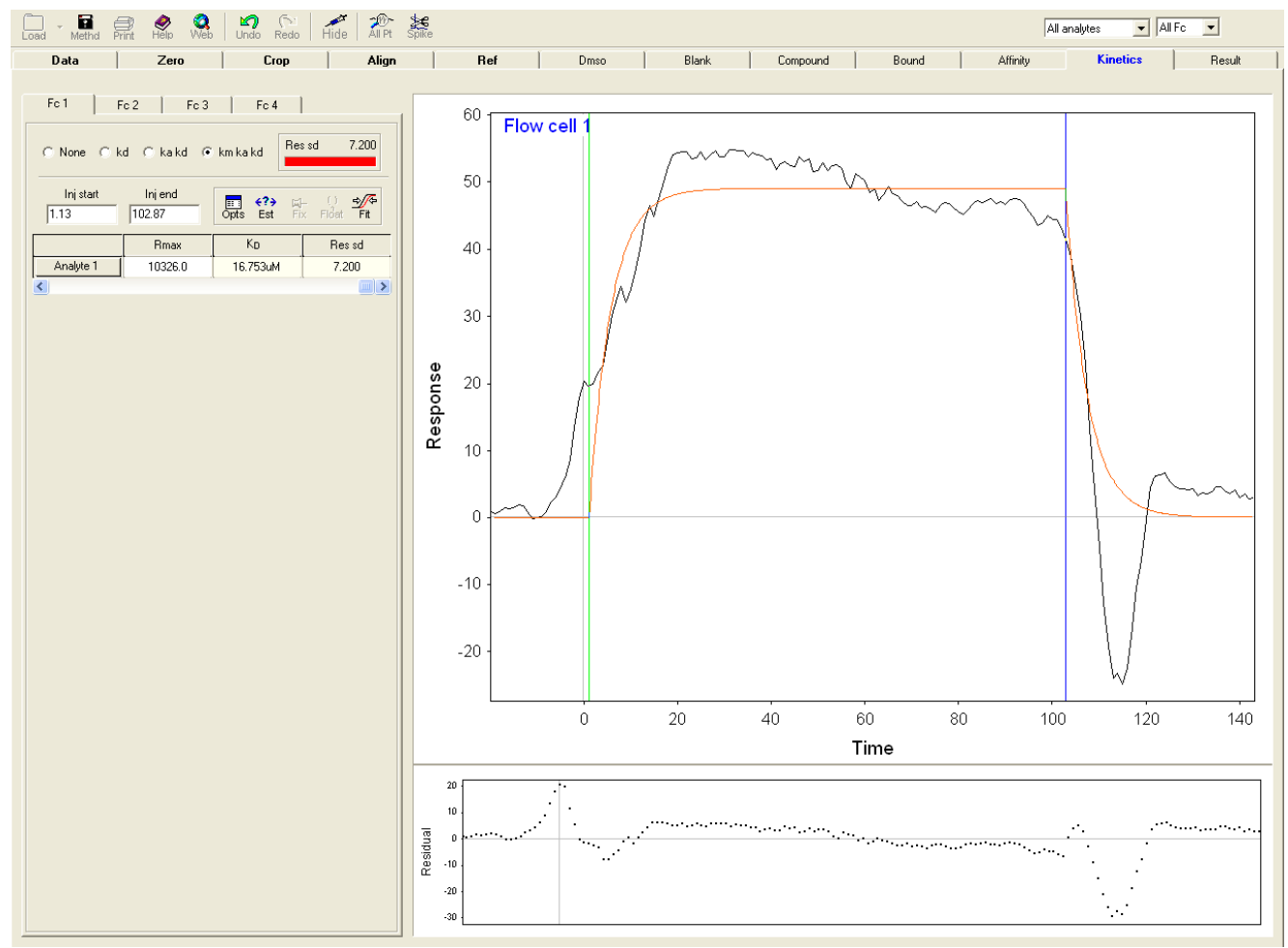

100nM antibody kinetic analysis 2 


\section{H. VEGF INFORMATION SHEET}

Syd Labs, Inc nww sydlabs com message@sydlabs com Tel 1-617-401-8149 Fax: 1-617-606-5019

\section{Recombinant Human VEGF 165}

Catalog No.: BP000027-GD1

Background

Vascular endothelial growth factor (VEGF), also known as vascular permeability factor (VPF) and VEGF-A, is a potent mediator of both angiogenesis and vasculogenesis in the fetus and adult $(1,2)$ It is a member of the platelet-derived growth factor (PDGF)/vascular endothelial growth factor (VEGF) family and often exists as a disulfide-linked homodimer (3). VEGF-A protein is a glycosylated mitogen that specifically acts on endothelial cells and has various effects, including mediating increased vascular permeability, inducing angiogenesis, vasculogenesis and endothelial cell growth, promoting cell migration, inhibiting apoptosis and tumor growth. VEGF-A protein is also a vasodilator that increases microvascular permeability, thus it was originally referred to as vascular permeability factor.

\section{Reference}

1. Leung. DW et al (1989) Science 246: 1306

2. Byrne, A.M. et al (2005) J Cell. Mol. Med. 9: 777

3. Robinson, C.J. and S.E. Stringer (2001) J. Cell. Sci. 114: 853

Source. HEK 293, Ala27-Arg191, Accession\# NP_001165097

$\mathrm{N}$-terminal sequence analysis: Met-Asn

Predicted molecular mass: $19.2 \mathrm{kDa}$ (monomer)

SDS-PAGE: 17 - $20 \mathrm{kDa}$, reducing conditions

Quality control: Measured in a cell proliferation assay using human umbilical vein endothelial cells (HUVEC). Conn, G. et al. (1990) Proc. Natl. Acad. Sci. USA 87:1323. The ED 50 for this effect is typically $1-5 \mathrm{ng} / \mathrm{mL}$

Formulation: $10 \mu \mathrm{g}$ per vial, lyophilized from sterile solution in PBS

Endotoxin Level $<10 \mathrm{EU}$ per $1 \mathrm{mg}$ of the protein by the LAL method.

Purity $>95 \%$, by SDS-PAGE and RP-HPLC.

Reconstitution: Dissolved in $135 \mu \mathrm{ldd} \mathrm{H}_{2} \mathrm{O}$ to the concentration of $1.5 \mathrm{mg} / \mathrm{ml}$. To further dilute the VEGF concentration under $10 \mu \mathrm{g} / \mathrm{ml}, 1 \%$ BSA is recommended

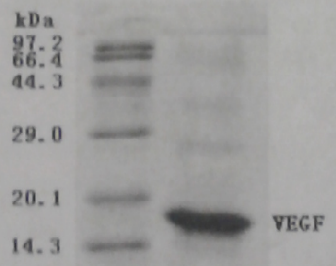

Shipping: The product is shipped at $4^{\circ} \mathrm{C}$. Upon receipt, store it immediately at the temperature recommended below

Stability \& storage:

Use a manual defrost freezer and avoid repeated freeze-thaw cycles.

12 months from date of receipt if stored at $-20^{\circ} \mathrm{C}$ to $-70^{\circ} \mathrm{C}$ as supplied

1 month after reconstitution if stored at $2^{\circ} \mathrm{C}$ to $8^{\circ} \mathrm{C}$ under sterile conditions.

The punty of recombinant bVEGF was determued by SDS-PAGE 3 months affer reconstitution if stored at $-20^{\circ} \mathrm{C}$ to $-70^{\circ} \mathrm{C}$ under sterile conditions 


\section{ANTIBOdy INFORMATION SHEET}

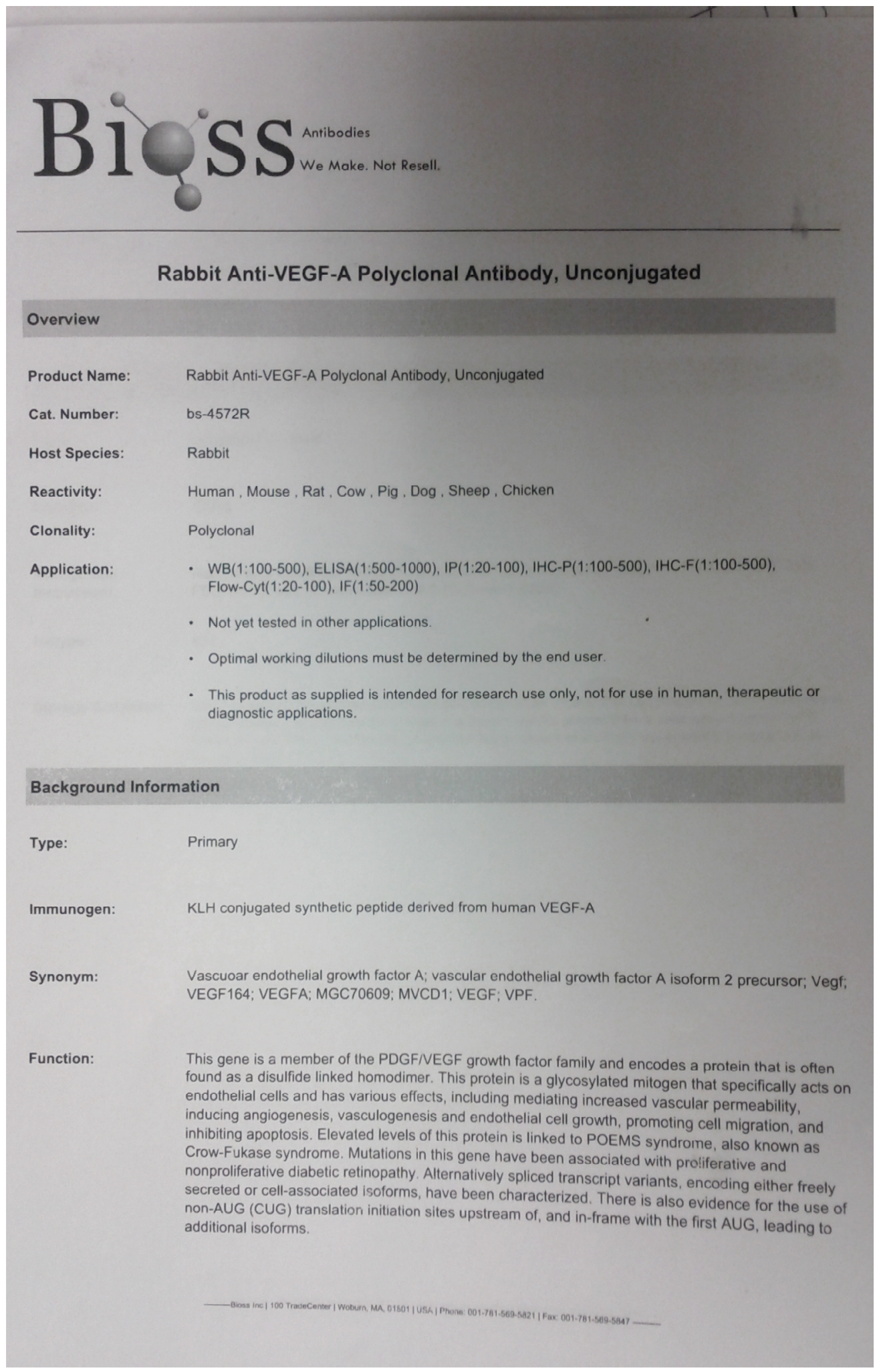




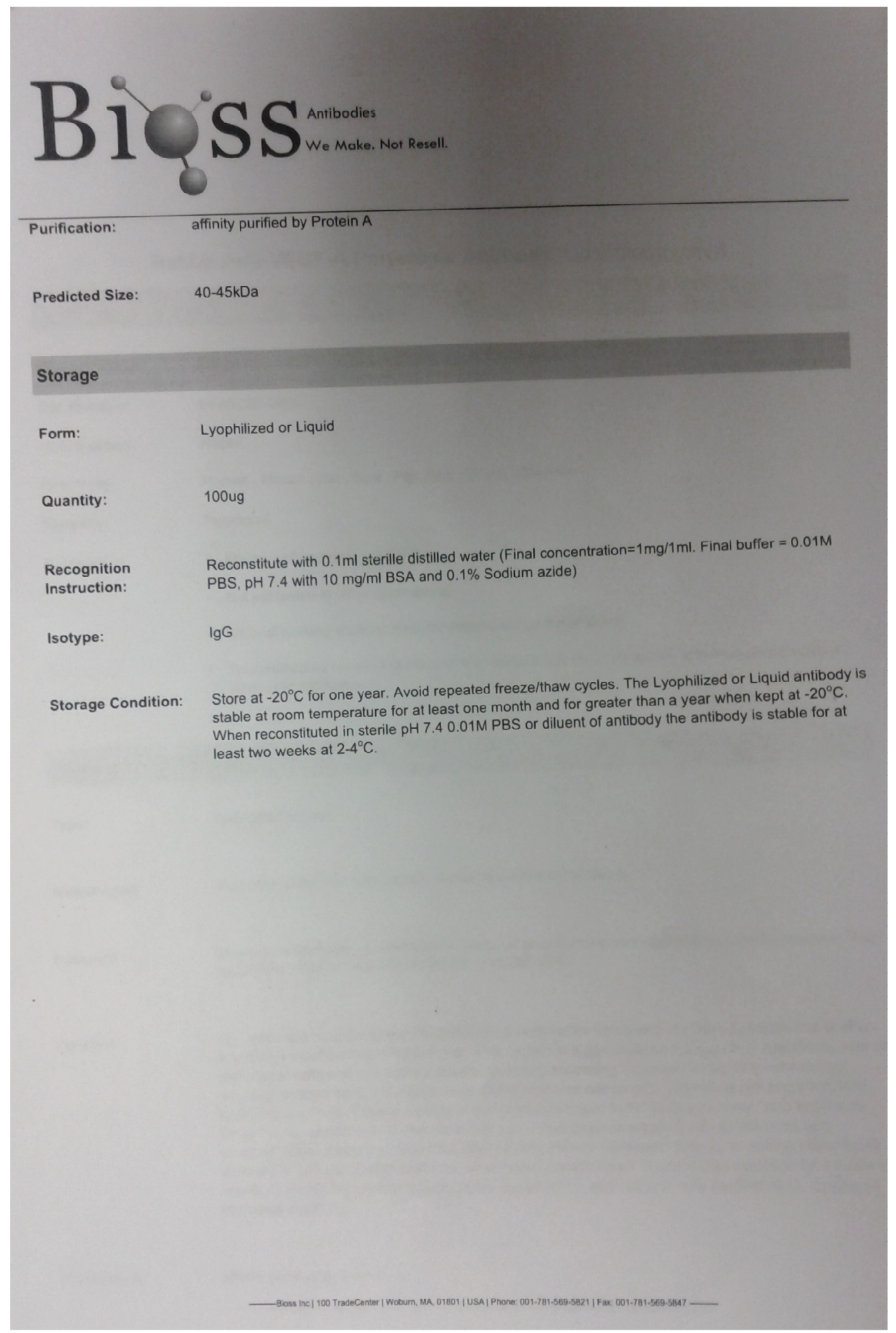




\section{J. BI ANalysis Software Protocol}

All data is from ethanol dilutions experiment from protocol except for Step 2 and Step 7.

Step 1: Open raw date curves from experiment. (Figure 1)

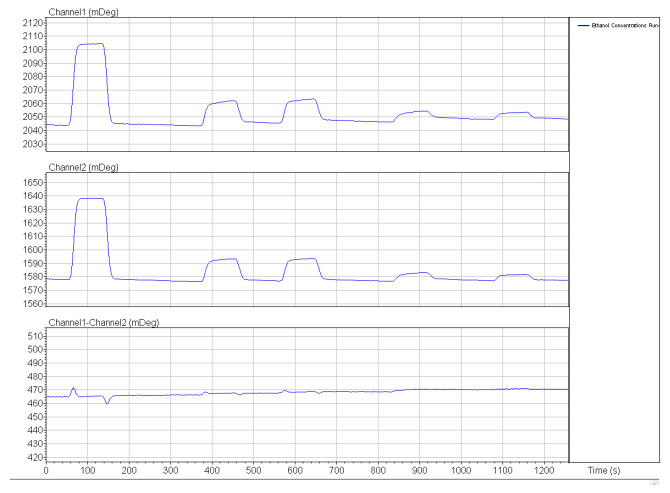

Figure 1

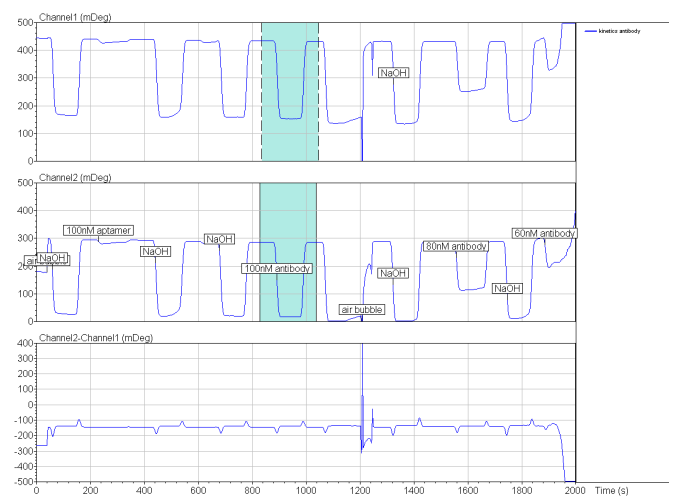

Figure 2
Step 2: Select curve and reference curve. (Figure 2)
Step 3: Enter concentrations of solutions injected. For example: if $30 \mathrm{nM}$ solution was used, enter $30 \mathrm{n}$. For example, if 3 dilutions were made, enter concentration into 'Bulk conc' and then the dilution factor, and then 1, 2, 3 for each concurrent dilution: bulk concentration $=1 \%(0.01)$ and dilutions of $1 / 4$ are made, enter 1 for $1 \%$ and 2 for $1 / 4$ dilution and 3 for $1 / 16$. (Figure 3)

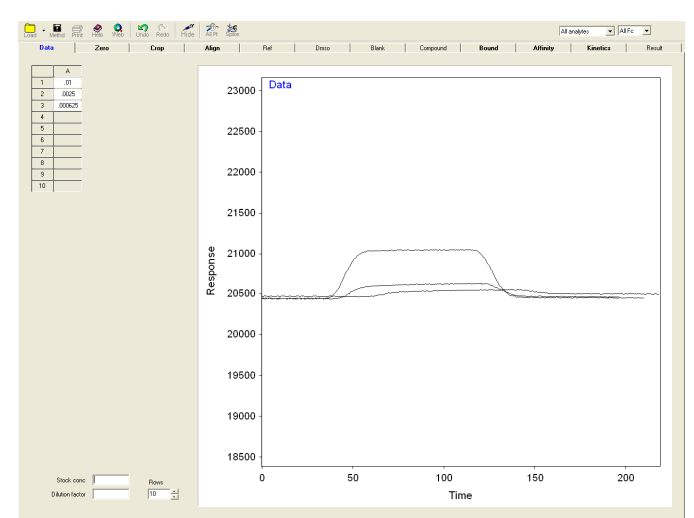

Figure 3 
Step 4: Zero the data by placing the green and then red lines over an area of the curve that is flat for each curve. (Figure 4)

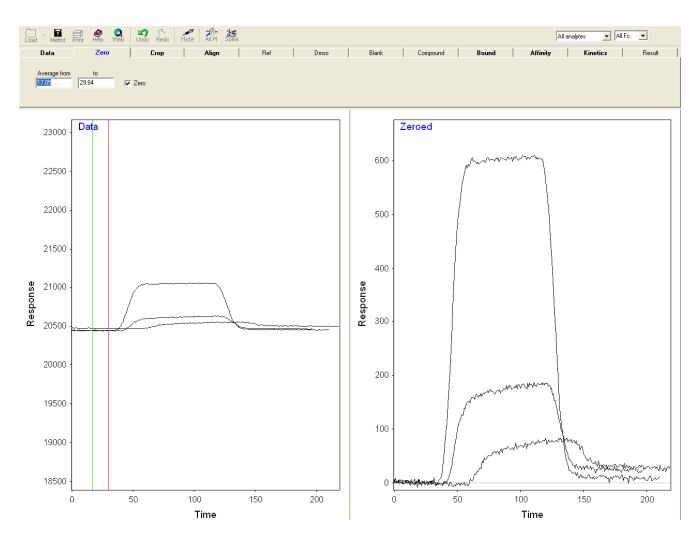

Figure 4

Step 5: Crop the data to remove excess from each side by moving the green line to crop at the beginning and the red line to crop at the end. (Figure 5)

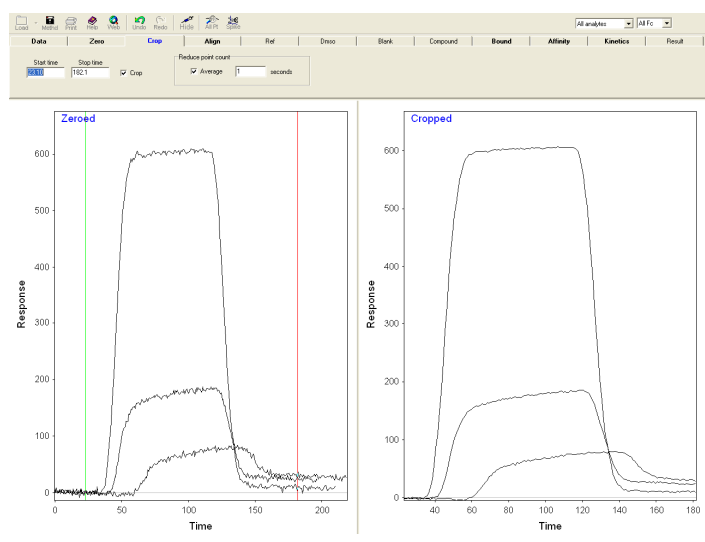

Figure 5

Step 6: Align the data by placing the green line at the beginning of the first curve and the red line at the peak of the last curve to remove any variation in time due to channels. (Figure 6)

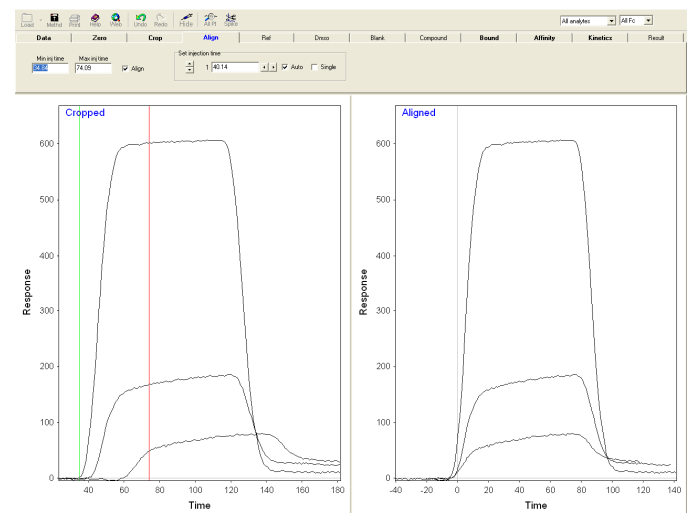

Figure 6 
Step 7: Subtract the reference channel from the channel where sample was bound (channel 1-channel 2 or channel 2channel 1). (Figure 7)

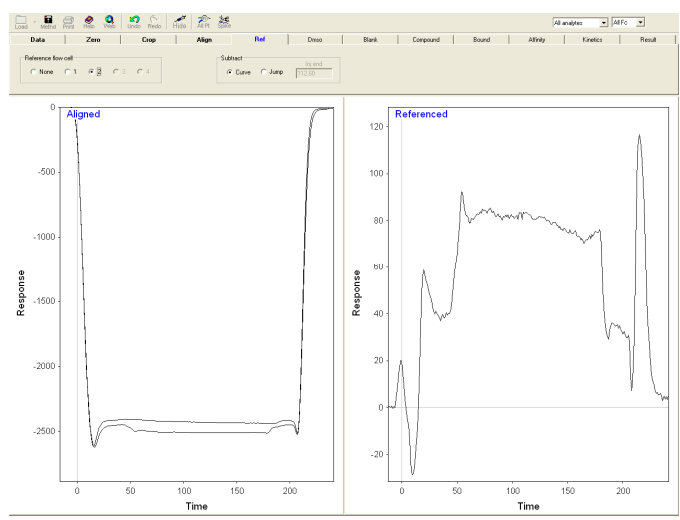

Figure 7

Step 8: Bound ligand is calculated by placing the green and red lines where each curve is at steady state. (Figure 8)

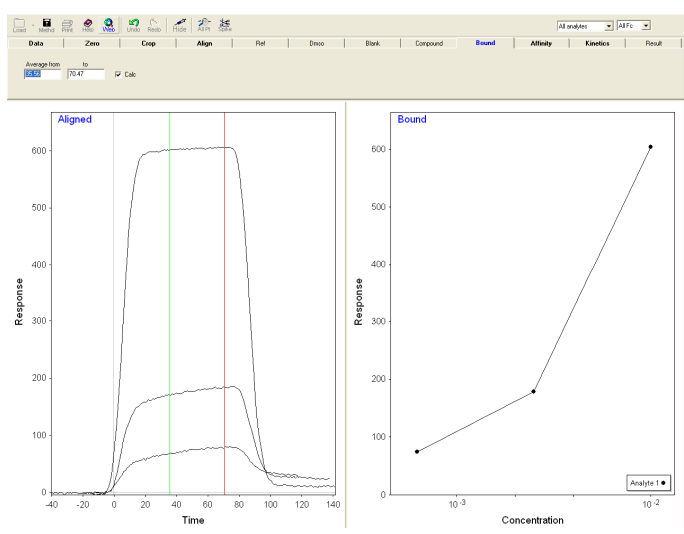

Figure 8

Step 9: Computer calculates the affinity, $\mathrm{K}_{\mathrm{D}}$ and $\mathrm{R}_{\mathrm{maz}}$. Also shows the curve of bound ligand at steady state verses the concentration. (Figure 9)

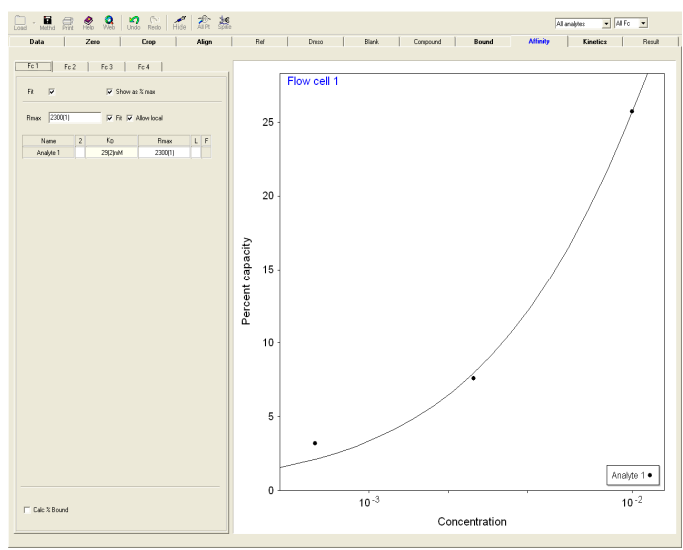

Figure 9 
Step 10: Fit the orange lines to the data curves and the kinetic data $K_{D}, k_{a}, k_{d}$, $R_{m a c}, k_{m}$ are calculated. How well the line fits the curve is shown by either a red or green bar above the results. The green line is placed where the curve begins ad the red line is placed where the steady state ends and the curve drops back down. (Figure 10)

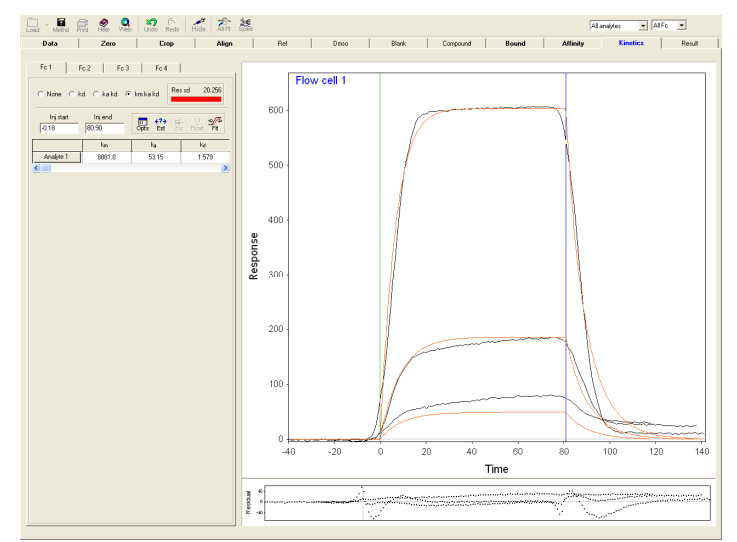

Figure 10

Step 11: Can then view all results on the Results pag 
